\title{
Heat asymptotics for nonminimal Laplace type operators and application to noncommutative tori
}

\author{
B. Iochum ${ }^{\mathrm{a}}, \mathrm{T} \cdot$ Masson $^{\mathrm{a}}$ \\ ${ }^{a}$ Centre de Physique Théorique \\ Aix Marseille Univ, Université de Toulon, CNRS, CPT, Marseille, France
}

\begin{abstract}
Let $P$ be a Laplace type operator acting on a smooth hermitean vector bundle $V$ of fiber $\mathbb{C}^{N}$ over a compact Riemannian manifold given locally by $P=-\left[g^{\mu \nu} u(x) \partial_{\mu} \partial_{\nu}+v^{\nu}(x) \partial_{\nu}+w(x)\right]$ where $u, v^{\nu}, w$ are $M_{N}(\mathbb{C})$-valued functions with $u(x)$ positive and invertible. For any $a \in \Gamma(\operatorname{End}(V))$, we consider the asymptotics $\operatorname{Tr}\left(a e^{-t P}\right) \underset{t \downarrow 0^{+}}{\sim} \sum_{r=0}^{\infty} a_{r}(a, P) t^{(r-d) / 2}$ where the coefficients $a_{r}(a, P)$ can be written locally as $a_{r}(a, P)(x)=\operatorname{tr}\left[a(x) \mathcal{R}_{r}(x)\right]$.

The computation of $\mathcal{R}_{2}$ is performed opening the opportunity to calculate the modular scalar curvature for noncommutative tori.

Keywords: Heat kernel, nonminimal operator, asymptotic heat trace, Laplace type operator, scalar curvature, noncommutative torus

PACS: 11.15.-q, 04.62.+v

2000 MSC: 58J35, 35J47, 81T13, 46L87
\end{abstract}

\section{Introduction}

As in [1], we consider a $d$-dimensional compact Riemannian manifold $(M, g)$ without boundary, together with a nonminimal Laplace type operator $P$ on a smooth hermitean vector bundle $V$ over $M$ of fiber $\mathbb{C}^{N}$ written locally as

$$
P:=-\left[g^{\mu \nu} u(x) \partial_{\mu} \partial_{\nu}+v^{\nu}(x) \partial_{\nu}+w(x)\right] .
$$

Here $u(x) \in M_{N}(\mathbb{C})$ is a positive and invertible matrix valued function and $v^{\nu}, w$ are $M_{N}(\mathbb{C})$ matrices valued functions. The operator is expressed in a local trivialization of $V$ over an open subset of $M$ which is also a chart on $M$ with coordinates $\left(x^{\mu}\right)$. This trivialization is such that the adjoint for the hermitean metric corresponds to the adjoint of matrices and the trace on endomorphisms on $V$ becomes the usual trace tr on matrices.

For any $a \in \Gamma(\operatorname{End}(V))$, we consider the asymptotics of the heat-trace

$$
\operatorname{Tr}\left(a e^{-t P}\right) \underset{t \downarrow 0^{+}}{\sim} \sum_{r=0}^{\infty} a_{r}(a, P) t^{(r-d) / 2} .
$$

Email addresses: bruno.iochum@cpt.univ-mrs.fr (B. Iochum), thierry.masson@cpt.univ-mrs.fr (T. Masson) 
where $\operatorname{Tr}$ is the operator trace. Each coefficient $a_{r}(a, P)$ can be written as

$$
a_{r}(a, P)=\int_{M} a_{r}(a, P)(x) \operatorname{dvol}_{g}(x)
$$

where $\operatorname{dvol}_{g}(x):=|g|^{1 / 2} \mathrm{~d} x$ with $|g|:=\operatorname{det}\left(g_{\mu \nu}\right)$. The functions $a_{r}(a, P)(x)$ can be evaluated (various techniques exist for that) and give expressions of the form

$$
a_{r}(a, P)(x)=\operatorname{tr}\left[a(x) \mathcal{R}_{r}(x)\right],
$$

where $\operatorname{tr}$ is the trace on matrices and $\mathcal{R}_{r}$ is a (local) section of $\operatorname{End}(V)$. The local section $\mathcal{R}_{r}$ of $\operatorname{End}(V)$ is uniquely defined by

$$
a_{r}(a, P)=\varphi\left(a \mathcal{R}_{r}\right)
$$

where $\varphi(a):=\int_{M} \operatorname{tr}[a(x)] \operatorname{dvol}_{g}(x)$ is the natural combined trace on the algebra of sections of End $(V)$ associated to $(M, g)$ (the integral) and $V$ (the matrix trace). The choice of this trace is not unique, and changing $\varphi$ changes $\mathcal{R}_{r}$. For instance, since $M$ is compact, one can normalize the integral so that the total volume of $M$ is 1 , and also the matrix trace such that the trace of the identity matrix is 1 . In that case, denoted by $\mathbb{1}$ the identity operator in $\Gamma(\operatorname{End}(V))$, the new combined trace $\varphi_{0}$ satisfies $\varphi_{0}(\mathbb{1})=1$. In Section $5 \varphi_{0}$ plays an important role since it corresponds to the unique normalized trace on the noncommutative torus algebra. Another possibility for the choice of $\varphi$ is to use a Riemannian metric on $M$ which is not the tensor $g$ in $P$, see Remark 2.5.

The aim of this paper is to present a way to compute $\mathcal{R}_{r}$ by adapting the techniques developed in [1]. These techniques were strongly motivated by a need in physics for explicit computations of $a_{r}(\mathbb{1}, P)$, see for instance $[2,3]$ and the reference in [1] for the existing results on the mathematical side. The idea behind the computation of $\mathcal{R}_{2}$ is to extract the real matrix content of the coefficient $a_{2}$ which is related to the scalar curvature of the manifold $M$.

In Section 2, two formulas are provided for $\mathcal{R}_{2}(x)$, both in local coordinates (Theorem 2.3) and in a covariant way (Theorem 2.4) in arbitrary dimension and detailed in low dimensions. In Section 3 , some direct applications are also provided, for instance to a conformal like transformed Laplacian. Section 4 is devoted to the details of the computations (see also the ancillary Mathematica [4] notebook file [5]).

In Section 5, another applications are given in noncommutative geometry. Namely, we compute the conformally deformed scalar curvature of rational noncommutative tori (NCT). Since at rational values $\theta=p / q$ of the deformation parameter, the algebras of the NCT are isomorphic to the continuous sections of a bundle over the ordinary tori with fiber in $M_{q}(\mathbb{C})$, they fit perfectly with our previous framework. The irrational case has been widely studied in $[6,7,8,9,10,11,12,13,14,15,16,17,18]$. The results presented in these papers can be written without explicit reference to the parameter $\theta$. In the rational case, our results confirm this property. Moreover, our method gives an alternative which avoids the theory of pseudodifferential calculus on the noncommutative tori introduced by Connes and Tretkoff $[19,6]$, see also [20]. In Appendix B, in order to confirm the results in [8, Theorem 5.2] and [11, Theorem 5.4], we perform the change of variables from $u$ to $\ln (u)$ and the change of operators from the left multiplication by $u$ to the conjugation by $u$, formalized as a rearrangement lemma (Lemma B.1).

\section{The method and the results}

In [1], the computation was done for the special case $a=\mathbb{1}$, for which a lot of simplifications can be used under the trace. We now show that the method described there can be adapted almost 
without any change to compute the quantities $\mathcal{R}_{r}$. Moreover, we present the method in a way that reduces the number of steps in the computations, using from the beginning covariant derivatives on the vector bundle $V$.

\subsection{Notations and preliminary results}

In order to start with the covariant form of $P$ (see [1, Section A.4]), let us introduce the following notations. We consider a covariant derivative $\nabla_{\mu}:=\partial_{\mu}+\eta\left(A_{\mu}\right)$, where $\eta$ is the representation of the Lie algebra of the gauge group of $V$ on any associated vector bundles (mainly $V$ and $\operatorname{End}(V)$ in the following). Let

$$
\alpha_{\mu}:=g_{\rho \sigma}\left(\partial_{\mu} g^{\rho \sigma}\right), \quad \alpha^{\mu}:=g^{\mu \nu} \alpha_{\nu}=g^{\mu \nu} g_{\rho \sigma}\left(\partial_{\nu} g^{\rho \sigma}\right), \quad \beta_{\mu}:=g_{\mu \sigma}\left(\partial_{\rho} g^{\rho \sigma}\right), \quad \beta^{\mu}:=g^{\mu \nu} \beta_{\nu}=\partial_{\nu} g^{\mu \nu} .
$$

The covariant form of $P$ associated to $\nabla$ (see [1, eq. (A.11)]) is given by

$$
\begin{aligned}
P & =-\left(|g|^{-1 / 2} \nabla_{\mu}|g|^{1 / 2} g^{\mu \nu} u \nabla_{\nu}+p^{\mu} \nabla_{\mu}+q\right) \\
& =-g^{\mu \nu} u \nabla_{\mu} \nabla_{\nu}-\left(p^{\nu}+g^{\mu \nu}\left(\nabla_{\mu} u\right)-\left[\frac{1}{2} \alpha^{\nu}-\beta^{\nu}\right] u\right) \nabla_{\nu}-q,
\end{aligned}
$$

where the last equality is obtained using $g^{\mu \nu} \frac{1}{2} \partial_{\mu} \ln |g|+\partial_{\mu} g^{\mu \nu}=-\left[\frac{1}{2} \alpha^{\nu}-\beta^{\nu}\right]$. Here, $u$ is as before, and $p^{\mu}, q$ are as $v^{\mu}, w$ from (1.1), except that they transform homogeneously in a change of trivialization of $V$. All these (local) functions are $M_{N}(\mathbb{C})$-valued (as local sections of $\operatorname{End}(V)$ ), so that $\eta$ is the adjoint representation:

$$
\nabla_{\mu} u=\partial_{\mu} u+\left[A_{\mu}, u\right], \quad \nabla_{\mu} p^{\nu}=\partial_{\mu} u+\left[A_{\mu}, p^{\nu}\right], \quad \nabla_{\mu} q=\partial_{\mu} u+\left[A_{\mu}, q\right] .
$$

Let us introduce the total covariant derivative $\widehat{\nabla}_{\mu}$, which combines $\nabla_{\mu}$ with the Levi-Civita covariant derivative induced by the metric $g$. It satisfies

$$
\begin{array}{llrl}
\widehat{\nabla}_{\mu} a^{\nu} & =\nabla_{\mu} a^{\nu}+\Gamma_{\mu \rho}^{\nu} a^{\rho}=\partial_{\mu} a^{\nu}+\left[A_{\mu}, a^{\nu}\right]+\Gamma_{\mu \rho}^{\nu} a^{\rho}, & \widehat{\nabla}_{\mu} g^{\alpha \beta} & =0, \\
\widehat{\nabla}_{\mu} b_{\nu} & =\nabla_{\mu} b_{\nu}-\Gamma_{\mu \nu}^{\rho} b_{\rho}=\partial_{\mu} b_{\nu}+\left[A_{\mu}, b_{\nu}\right]-\Gamma_{\mu \nu}^{\rho} b_{\rho}, & \widehat{\nabla}_{\mu} g_{\alpha \beta}=0,
\end{array}
$$

for any $\operatorname{End}(V)$-valued tensors $a^{\nu}$ and $b_{\nu}$, where $\Gamma_{\mu \rho}^{\nu}$ are the Christoffel symbols of $g$. Let us store the following relations:

$$
\begin{aligned}
\widehat{\nabla}_{\mu} u & =\nabla_{\mu} u \\
g^{\mu \nu} \widehat{\nabla}_{\mu} \widehat{\nabla}_{\nu} u & =g^{\mu \nu}\left(\nabla_{\mu} \nabla_{\nu} u-\Gamma_{\mu \nu}^{\rho} \nabla_{\rho} u\right)=g^{\mu \nu} \nabla_{\mu} \nabla_{\nu} u-\left[\frac{1}{2} \alpha^{\mu}-\beta^{\mu}\right] \nabla_{\mu} u, \\
\widehat{\nabla}_{\mu} p^{\mu} & =\nabla_{\mu} p^{\mu}-\frac{1}{2} g_{\alpha \beta}\left(\partial_{\mu} g^{\alpha \beta}\right) p^{\mu}=\nabla_{\mu} p^{\mu}-\frac{1}{2} \alpha_{\mu} p^{\mu} .
\end{aligned}
$$

Using $\frac{1}{2} \alpha^{\rho}-\beta^{\rho}=g^{\mu \nu} \Gamma_{\mu \nu}^{\rho}$, one then has

$$
P=-\left(g^{\mu \nu} \widehat{\nabla}_{\mu} u \widehat{\nabla}_{\nu}+p^{\nu} \widehat{\nabla}_{\nu}+q\right)=-g^{\mu \nu} u \widehat{\nabla}_{\mu} \widehat{\nabla}_{\nu}-\left[p^{\nu}+g^{\mu \nu}\left(\widehat{\nabla}_{\mu} u\right)\right] \widehat{\nabla}_{\nu}-q .
$$

Notice that in these expressions the total covariant derivative $\widehat{\nabla}_{\nu}$ (which is the first to act) will never apply to a tensor valued section of $V$, so that it could be reduced to the covariant derivative $\nabla_{\nu}$.

The writing of $P$ in terms of a covariant derivative $\nabla$ is of course not unique: 
Proposition 2.1 Let $\nabla_{\mu}^{\prime}=\nabla_{\mu}+\eta\left(\phi_{\mu}\right)$ be another covariant derivative on $V$. Then

$$
P=-\left(g^{\mu \nu} \widehat{\nabla}_{\mu}^{\prime} u \widehat{\nabla}_{\nu}^{\prime}+p^{\prime \nu} \widehat{\nabla}_{\nu}^{\prime}+q^{\prime}\right)
$$

with

$$
p^{\prime \nu}=p^{\nu}-g^{\mu \nu}\left(u \phi_{\mu}+\phi_{\mu} u\right), \quad q^{\prime}=q-g^{\mu \nu}\left(\widehat{\nabla}_{\mu} u \phi_{\nu}\right)+g^{\mu \nu} u \phi_{\mu} \phi_{\nu}-p^{\mu} \phi_{\mu} .
$$

In this proposition, $\phi_{\mu}$ is as $p^{\mu}$ : it transforms homogeneously in a change of trivializations of $V$.

ProOF This is a direct computation using relations like $\widehat{\nabla}_{\mu}^{\prime} u=\widehat{\nabla}_{\mu} u+\left[\phi_{\mu}, u\right]$ and $\widehat{\nabla}_{\mu}^{\prime} \phi_{\nu}=\widehat{\nabla}_{\mu} \phi_{\nu}+$ $\left[\phi_{\mu}, \phi_{\nu}\right]$ in (2.5) and comparing with (2.4).

Corollary 2.2 There is a unique covariant derivative $\nabla$ such that $p^{\mu}=0$. This implies that we can always write $P$ in the reduced form

$$
P=-\left(g^{\mu \nu} \widehat{\nabla}_{\mu} u \widehat{\nabla}_{\nu}+q\right)
$$

Proof The first part of (2.6) can be solved in $\phi_{\mu}$ for the condition $p^{\prime \nu}=0$. Indeed, using results in [21], the positivity and invertibility of $u$ implies that for any $\nu$, the equation $u\left(g^{\mu \nu} \phi_{\mu}\right)+\left(g^{\mu \nu} \phi_{\mu}\right) u=p^{\nu}$ has a unique solution given by

$$
g^{\mu \nu} \phi_{\mu}=\frac{1}{2} \int_{-\infty}^{+\infty} \frac{u^{i t-1 / 2} p^{\nu} u^{-i t-1 / 2}}{\cosh (\pi t)} d t .
$$

So, given any covariant derivative to which $p^{\nu}$ is associated as in (2.1), we can shift this covariant derivative with the above solution $\phi_{\mu}$ to impose $p^{\prime \nu}=0$.

This result extends the one in [22, Section 1.2.1], which is a key ingredient of the method used there. In the following, we could have started with $P$ written as in (2.7). But, on one hand, we will see that this is not necessary to get $\mathcal{R}_{r}$ in terms on $u, p^{\mu}, q$ (at least for $r=2$ ). On the other hand, we will see in Section 5 that the covariant derivative which is naturally given by the geometric framework of the rational noncommutative torus does not implies $p^{\mu}=0$, and we will then apply directly the most general result. Obviously, it could be possible to first establish our result for the reduced expression (2.7) and then to go to the general result using Prop. 2.1. But this would complicate unnecessarily the presentation of the method and our results.

\subsection{The method}

The method described in [1] starts with $P$ written as $P=-g^{\mu \nu} u \partial_{\mu} \partial_{\nu}-v^{\mu} \partial_{\mu}-w$ and leads to $-P\left(e^{i x \xi} f\right)=-e^{i x \xi}[H+K+P] f$ where $H=g^{\mu \nu} u \xi_{\mu} \xi_{\nu}$ and $K=-i \xi_{\mu}\left(v^{\mu}+2 g^{\mu \nu} u \partial_{\nu}\right)$.

This can be generalized for a covariant writing of $P$. Using (2.1), one gets

$$
\begin{aligned}
-P\left(e^{i x \xi} f\right)= & e^{i x \xi}\left[-g^{\mu \nu} u \xi_{\mu} \xi_{\nu}+i \xi_{\mu}\left(p^{\mu}+g^{\mu \nu}\left(\nabla_{\nu} u\right)-\left[\frac{1}{2} \alpha^{\nu}-\beta^{\nu}\right] u+2 g^{\mu \nu} u \nabla_{\nu}\right)\right. \\
& \left.g^{\mu \nu} u \nabla_{\mu} \nabla_{\nu}+\left(p^{\nu}+g^{\mu \nu}\left(\nabla_{\mu} u\right)-\left[\frac{1}{2} \alpha^{\nu}-\beta^{\nu}\right] u\right) \nabla_{\nu}+q\right] f \\
= & -e^{i x \xi}[H+K+P] f,
\end{aligned}
$$

with

$$
H:=g^{\mu \nu} u \xi_{\mu} \xi_{\nu}, \quad K:=-i \xi_{\mu}\left(p^{\mu}+g^{\mu \nu}\left(\nabla_{\nu} u\right)-\left[\frac{1}{2} \alpha^{\mu}-\beta^{\mu}\right] u+2 g^{\mu \nu} u \nabla_{\nu}\right)
$$


These relations look like the expressions of $H$ and $K$ given above (see [1, eq. (1.6), (1.7)]) with the replacements

$$
\partial_{\mu} \mapsto \nabla_{\mu}, \quad v^{\mu} \mapsto p^{\mu}+g^{\mu \nu}\left(\nabla_{\nu} u\right)-\left[\frac{1}{2} \alpha^{\mu}-\beta^{\mu}\right] u, \quad w \mapsto q
$$

As in [1], we have $\operatorname{Tr}\left[a e^{-t P}\right]=\int \mathrm{d} x \operatorname{tr}[a(x) K(t, x, x)]$ with

$$
K(t, x, x)=\frac{1}{(2 \pi)^{d}} \int \mathrm{d} \xi e^{-i x \cdot \xi}\left(e^{-t P} e^{i x \cdot \xi}\right)=\frac{1}{(2 \pi)^{d}} \int \mathrm{d} \xi e^{-t(H+K+P)} \mathbb{1}=\frac{1}{t^{d / 2}} \frac{1}{(2 \pi)^{d}} \int \mathrm{d} \xi e^{-H-\sqrt{t} K-t P} \mathbb{1} .
$$

Here $\mathbb{1}$ is the constant 1 -valued function. Notice that $K(t, x, x)$ is a density, and that $|g|^{-1 / 2} K(t, x, x)$ is a true function on $M$. Using the Lebesgue measure $\mathrm{d} x$ instead of $\operatorname{dvol}_{g}(x)$ is convenient to establish the previous relation which uses Fourier transforms (this point has not been emphasized in [1]).

The asymptotics expansion is obtained by the Volterra series

$$
e^{A+B}=e^{A}+\sum_{k=1}^{\infty} \int_{\Delta_{k}} \mathrm{~d} s e^{\left(1-s_{1}\right) A} B e^{\left(s_{1}-s_{2}\right) A} \cdots e^{\left(s_{k-1}-s_{k}\right) A} B e^{s_{k} A} .
$$

where

$$
\Delta_{k}:=\left\{s=\left(s_{1}, \ldots, s_{k}\right) \in \mathbb{R}_{+}^{k} \mid 0 \leq s_{k} \leq s_{k-1} \leq \cdots \leq s_{2} \leq s_{1} \leq 1\right\} \text { and } \Delta_{0}:=\varnothing \text { by convention. }
$$

For $A=-H$ and $B=-\sqrt{t} K-t P$, one gets

$$
e^{-H-\sqrt{t} K-t P} \mathbb{1}=e^{-H}+\sum_{k=1}^{\infty}(-1)^{k} f_{k}[(\sqrt{t} K+t P) \otimes \cdots \otimes(\sqrt{t} K+t P)]
$$

with

$$
\begin{aligned}
f_{k}(\xi)\left[B_{1} \otimes \cdots \otimes B_{k}\right] & :=\int_{\Delta_{k}} \mathrm{~d} s e^{\left(s_{1}-1\right) H(\xi)} B_{1} e^{\left(s_{2}-s_{1}\right) H(\xi)} B_{2} \cdots B_{k} e^{-s_{k} H(\xi)}, \\
f_{0}(\xi)[\lambda] & :=\lambda e^{-H(\xi)},
\end{aligned}
$$

where $B_{i}$ are matrix-valued differential operators in $\nabla_{\mu}$ depending on $x$ and (linearly in) $\xi$, and $\lambda \in \mathbb{C}$. Collecting the powers of $\sqrt{t}$, one gets

$$
\operatorname{Tr}\left[a e^{-t P}\right] \underset{t \downarrow 0}{\simeq} t^{-d / 2} \sum_{r=0}^{\infty} a_{r}(a, P) t^{r / 2}
$$

Each $a_{r}(a, P)$ contains an integration along $\xi$, which kills all the terms in odd power in $\sqrt{t}$ since $K$ is linear in $\xi$ while $H$ is quadratic in $\xi: a_{2 n+1}(a, P)=0$ for any $n \in \mathbb{N}$. For instance, the first two non-zero local coefficients are ${ }^{1}$

$$
\begin{aligned}
a_{0}(a, P)(x) & =\frac{|g|^{-1 / 2}}{(2 \pi)^{d}} \operatorname{tr}\left[a(x) \int \mathrm{d} \xi e^{-H(x, \xi)}\right] \\
a_{2}(a, P)(x) & =\frac{|g|^{-1 / 2}}{(2 \pi)^{d}} \operatorname{tr}\left[a(x) \int \mathrm{d} \xi \int_{\Delta_{2}} \mathrm{~d} s e^{\left(s_{1}-1\right) H} K e^{\left(s_{2}-s_{1}\right) H} K e^{-s_{2} H}\right] \\
& -\frac{1}{(2 \pi)^{d}} \operatorname{tr}\left[a(x) \int \mathrm{d} \xi \int_{\Delta_{1}} \mathrm{~d} s e^{\left(s_{1}-1\right) H} P e^{-s_{1} H}\right]
\end{aligned}
$$

\footnotetext{
${ }^{1}$ Notice the change with convention in [1] $: a_{2 r}$ here corresponds to $a_{r}$ in [1].
} 
(remark the coefficient $|g|^{-1 / 2}$ added here to be compatible with (1.3)).

The strategy to compute these coefficient is twofold. First, we get rid of the $\nabla_{\mu}$ 's in the arguments $B_{i}$. This is done using [1, Lemma 2.1], which can be applied here since $\nabla_{\mu}$ is a derivation: by iteration of the relation

$$
\begin{aligned}
f_{k}(\xi)\left[B_{1} \otimes \cdots \otimes B_{i} \nabla_{\mu} \otimes \cdots \otimes B_{k}\right] & =\sum_{j=i+1}^{k} f_{k}(\xi)\left[B_{1} \otimes \cdots \otimes\left(\nabla_{\mu} B_{j}\right) \otimes \cdots \otimes B_{k}\right] \\
& -\sum_{j=i}^{k} f_{k+1}(\xi)\left[B_{1} \otimes \cdots \otimes B_{j} \otimes\left(\nabla_{\mu} H\right) \otimes B_{j+1} \otimes \cdots \otimes B_{k}\right],
\end{aligned}
$$

we transform each original term into a sum of operators acting on arguments of the form $B_{1} \otimes \cdots \otimes$ $B_{k}=\mathbb{B}_{k}^{\mu_{1} \ldots \mu_{\ell}} \xi_{\mu_{1}} \cdots \xi_{\mu_{\ell}}$ (for different values of $k$ ) where now all the $B_{i}$ are matrix-valued functions (of $x$ and $\xi$ ), or, equivalently, the $\mathbb{B}_{k}^{\mu_{1} \ldots \mu_{\ell}}$ are $M_{N}(\mathbb{C})^{\otimes^{k}}$-valued functions (of $x$ only).

The second step of the strategy is to compute the operators applied to the arguments $\mathbb{B}_{k}^{\mu_{1} \ldots \mu_{\ell}}$. They all look like

$$
\frac{1}{(2 \pi)^{d}} \int \mathrm{d} \xi \xi_{\mu_{1}} \cdots \xi_{\mu_{\ell}} f_{k}(\xi)\left[\mathbb{B}_{k}^{\mu_{1} \ldots \mu_{\ell}}\right] \in M_{N}(\mathbb{C})
$$

where the $f_{k}(\xi)$ are defined by (2.12) and depend only on $u$ through $H$. As shown in [1], these operators are related to operators $T_{k, p}(x): M_{N}(\mathbb{C})^{\otimes^{k+1}} \rightarrow M_{N}(\mathbb{C})^{\otimes^{k+1}}$ defined by

$$
\begin{aligned}
T_{k, p}(x) & :=\frac{1}{(2 \pi)^{d}} \int_{\Delta_{k}} \mathrm{~d} s \int \mathrm{d} \xi \xi_{\mu_{1}} \cdots \xi_{\mu_{2 p}} e^{-\|\xi\|^{2} C_{k}(s, u(x))} \\
T_{0,0}(x) & :=\frac{1}{(2 \pi)^{d}} \int \mathrm{d} \xi e^{-\|\xi\|^{2} u(x)} \in M_{N}(\mathbb{C})
\end{aligned}
$$

where $\|\xi\|^{2}:=g^{\mu \nu} \xi_{\mu} \xi_{\nu}$ and the $C_{k}(s, A): M_{N}(\mathbb{C})^{\otimes^{k+1}} \rightarrow M_{N}(\mathbb{C})^{\otimes^{k+1}}$ are the operators

$$
\begin{array}{r}
C_{k}(s, A)\left[B_{0} \otimes B_{1} \otimes \cdots \otimes B_{k}\right]=\left(1-s_{1}\right) B_{0} A \otimes B_{1} \otimes \cdots \otimes B_{k} \\
+\left(s_{1}-s_{2}\right) B_{0} \otimes B_{1} A \otimes \cdots \otimes B_{k} \\
+\cdots+s_{k} B_{0} \otimes B_{1} \otimes \cdots \otimes B_{k} A .
\end{array}
$$

Denote by $\mathbf{m}: M_{N}(\mathbb{C})^{\otimes^{k+1}} \rightarrow M_{N}(\mathbb{C}), B_{0} \otimes B_{1} \otimes \cdots \otimes B_{k} \mapsto B_{0} B_{1} \cdots B_{k}$ the matrix multiplication, then

$$
\frac{1}{(2 \pi)^{d}} B_{0} \int \mathrm{d} \xi \xi_{\mu_{1}} \cdots \xi_{\mu_{\ell}} f_{k}(\xi)\left[B_{1} \otimes \cdots \otimes B_{k}\right]=\mathbf{m} \circ T_{k, p}(x)\left[B_{0} \otimes B_{1} \otimes \cdots \otimes B_{k}\right]
$$

so that each function $a_{r}(a, P)(x)$ is expressed formally as a sum

$$
a_{r}(a, P)(x)=|g|^{-1 / 2} \sum \operatorname{tr}\left[\mathbf{m} \circ T_{k, p}(x)\left[a(x) \otimes B_{1}(x) \otimes \cdots \otimes B_{k}(x)\right]\right] .
$$

This sum comes form the collection of the original terms in $K$ and $P$ producing the power $t^{r / 2}$ and the application of $[1$, Lemma 2.1] i.e. (2.13). This sum relates the $r$ on the LHS to the possible couples $(k, p)$ on the RHS. The $B_{i}$ are matrix-valued functions (of $x$ ) expressed in terms of the original constituents of $H, K$, and $P$ and their covariant derivatives.

Let us mention here how the procedure introduced in [1] is adapted to the situation where we have the left factor $a(x)$ : in [1], the relation between the $T_{k, p}(x)$ and the $f_{k}(\xi)$ used a trick which 
consist to add a $B_{0}=\mathbb{1}$ argument in front of $B_{1} \otimes \cdots \otimes B_{k}$ (the purpose of the $\kappa$ map defined in $[1])$. Here, $\mathbb{1}$ is simply replaced by $a(x)$. But, since

$$
\mathbf{m} \circ T_{k, p}(x)\left[B_{0} \otimes B_{1}(x) \otimes \cdots \otimes B_{k}(x)\right]=B_{0} \mathbf{m} \circ T_{k, p}(x)\left[\mathbb{1} \otimes B_{1}(x) \otimes \cdots \otimes B_{k}(x)\right],
$$

it is now easy to propose an expression for the factor $\mathcal{R}_{r}$ as a sum

$$
\mathcal{R}_{r}=|g|^{-1 / 2} \sum \mathbf{m} \circ T_{k, p}(x)\left[\mathbb{1} \otimes B_{1}(x) \otimes \cdots \otimes B_{k}(x)\right] .
$$

One of the main result of [1] is to express the operators $T_{k, p}$ in terms of universal functions through a functional calculus relation involving the spectrum of $u$ (these relations take place at any fixed value of $x \in M$, that we omit from now on). For $r_{i}>0, \alpha \in \mathbb{R}$, and $k \in \mathbb{N}$, let

$$
\begin{aligned}
I_{\alpha, k}\left(r_{0}, r_{1}, \ldots, r_{k}\right) & :=\int_{\Delta_{k}} \mathrm{~d} s\left[\left(1-s_{1}\right) r_{0}+\left(s_{1}-s_{2}\right) r_{1}+\cdots+s_{k} r_{k}\right]^{-\alpha} \\
& =\int_{\Delta_{k}} \mathrm{~d} s\left[r_{0}+s_{1}\left(r_{1}-r_{0}\right)+\cdots+s_{k}\left(r_{k}-r_{k-1}\right)\right]^{-\alpha}
\end{aligned}
$$

so that

$$
I_{\alpha, k}\left(r_{0}, \ldots, r_{0}\right)=\frac{1}{k !} r_{0}^{-\alpha}
$$

In these functions, the arguments $r_{i}>0$ are in the spectrum of the positive matrix $u$.

Denote by $R_{i}(A): M_{N}(\mathbb{C})^{\otimes^{k+1}} \rightarrow M_{N}(\mathbb{C})^{\otimes^{k+1}}$ the right multiplication on the $i$-th factor

$$
R_{i}(A)\left[B_{0} \otimes B_{1} \otimes \cdots \otimes B_{k}\right]:=B_{0} \otimes B_{1} \otimes \cdots \otimes B_{i} A \otimes \cdots \otimes B_{k},
$$

then

$$
T_{k, p}=g_{d} G(g)_{\mu_{1} \ldots \mu_{2 p}} I_{d / 2+p, k}\left(R_{0}(u), R_{1}(u), \ldots, R_{k}(u)\right)
$$

with

$$
\begin{aligned}
g_{d} & :=\frac{1}{(2 \pi)^{d}} \int_{\mathbb{R}^{d}} \mathrm{~d} \xi e^{-|\xi|_{g(x)}^{2}}=\frac{|g|^{1 / 2}}{2^{d} \pi^{d / 2}}, \\
G(g)_{\mu_{1} \ldots \mu_{2 p} p} & :=\frac{1}{(2 \pi)^{d} g_{d}} \int \mathrm{d} \xi \xi_{\mu_{1}} \cdots \xi_{\mu_{2 p}} e^{-g^{\alpha \beta} \xi_{\alpha} \xi_{\beta}} \\
& =\frac{1}{2^{2 p} p !}\left(\sum_{\rho \in S_{2 p}} g_{\mu_{\rho(1)} \mu_{\rho(2)}} \cdots g_{\mu_{\rho(2 p-1)} \mu_{\rho(2 p)}}\right)=\frac{(2 p) !}{2^{2 p} p !} g_{\left(\mu_{1} \mu_{2}\right.} \cdots g_{\left.\mu_{2 p-1} \mu_{2 p}\right)},
\end{aligned}
$$

where $S_{2 p}$ is the symmetric group of permutations on $2 p$ elements and the parenthesis in the index of $g$ is the complete symmetrization over all indices. Notice that the factor $|g|^{1 / 2}$ in $g_{d}$ simplifies with the factor $|g|^{-1 / 2}$ in $(2.16)$.

The universal functions $I_{\alpha, k}$ have been studied in [1, Section 3]. They satisfy a recursive formula valid for $1 \neq \alpha \in \mathbb{R}$ and $k \in \mathbb{N}^{*}$ :

$$
I_{\alpha, k}\left(r_{0}, \ldots, r_{k}\right)=\frac{1}{(\alpha-1)}\left(r_{k-1}-r_{k}\right)^{-1}\left[I_{\alpha-1, k-1}\left(r_{0}, \ldots, r_{k-2}, r_{k}\right)-I_{\alpha-1, k-1}\left(r_{0}, \ldots, r_{k-1}\right)\right] .
$$

It is possible to give some expressions for the $I_{\alpha, k}$ for any $(\alpha, k)$. They depend on the parity of $d$. For $d$ even, the main results are that $I_{n, k}$ are Laurent polynomials for $\mathbb{N} \ni n=(d-r) / 2+k \geq k+1$ $(d \geq r+2)$ and $k \in \mathbb{N}^{*}$, while they exhibit a more complicated expression in terms of log functions for $\mathbb{N} \ni n=(d-r) / 2+k \leq k(d \leq r)$. For $d$ odd, the $I_{n, k}$ can be expressed in terms of square roots of the $r_{i}$, but without an a priori general expression. 
The recursive formula (2.18) can be used to write any $I_{\alpha, k}$ appearing in the computation of the operators $T_{k, p}$ in terms of $I_{\alpha-k+1,1}$. The case $\alpha=1$ appears in dimension $d=2$ : the fundamental spectral function is $I_{1,1}$, and a direct computation shows that

$$
I_{1,1}=\frac{\ln \left(r_{0}\right)-\ln \left(r_{1}\right)}{r_{0}-r_{1}}
$$

Using $\frac{x}{e^{x}-1}=\sum_{n=0}^{\infty} \frac{B_{n}}{n !} x^{n}$, where $B_{n}$ are the Bernoulli numbers, one gets, with $x=\ln \left(r_{0}\right)-\ln \left(r_{1}\right)$,

$$
r_{1} I_{1,1}\left(r_{0}, r_{1}\right)=\sum_{n=0}^{\infty} \frac{B_{n}}{n !}\left[\ln \left(r_{0}\right)-\ln \left(r_{1}\right)\right]^{n}
$$

A relation between the Bernoulli numbers and $a_{2}(a, P)$ has already been noticed in the computation of the modular curvature for the noncommutative two torus in [7] (see Section 5).

\subsection{The results for $a_{2}(a, P)$}

In the following, we restrict ourselves to the computation of $a_{2}(a, P)$. This section gives the main results of the paper. The computations are detailed in Section 4.

Let us introduce the following notation. For any $x \in M$, denote by $r_{i}=r_{i}(x)>0$ an element in the (discrete) spectrum $\operatorname{sp}(u)$ of $u=u(x)$ and by $E_{r_{i}}=E_{r_{i}}(x)$ the associated projection of $u$. This implies that

$$
u=\sum_{r_{0} \in \operatorname{sp}(u)} r_{0} E_{r_{0}}=r_{0} E_{r_{0}}
$$

where in the last expression we omit the summation over $r_{0}$, as will be the case in many expressions given in the following. Notice that $\mathbb{1}=\sum_{r_{0} \in \operatorname{sp}(u)} E_{r_{0}}$ and $E_{r_{0}} E_{r_{1}}=\delta_{r_{0}, r_{1}} E_{r_{0}}$.

Theorem 2.3 For $P$ given by $(1.1), a_{2}(a, P)(x)=\operatorname{tr}\left[a(x) \mathcal{R}_{2}(x)\right]$ with

$$
\begin{aligned}
\mathcal{R}_{2}=\frac{1}{2^{d} \pi^{d / 2}}[ & \alpha r_{0}^{-d / 2+1} E_{r_{0}}+F_{\partial u}^{\mu}\left(r_{0}, r_{1}\right) E_{r_{0}}\left(\partial_{\mu} u\right) E_{r_{1}} \\
& +g^{\mu \nu} F_{\partial \partial u}\left(r_{0}, r_{1}\right) E_{r_{0}}\left(\partial_{\mu} \partial_{\nu} u\right) E_{r_{1}}+g^{\mu \nu} F_{\partial u, \partial u}\left(r_{0}, r_{1}, r_{2}\right) E_{r_{0}}\left(\partial_{\mu} u\right) E_{r_{1}}\left(\partial_{\nu} u\right) E_{r_{2}} \\
& +F_{w}\left(r_{0}, r_{1}\right) E_{r_{0}} w E_{r_{1}}+F_{v, \mu}\left(r_{0}, r_{1}\right) E_{r_{0}} v^{\mu} E_{r_{1}} \\
& +F_{v, \partial u}\left(r_{0}, r_{1}, r_{2}\right) E_{r_{0}} v^{\mu} E_{r_{1}}\left(\partial_{\mu} u\right) E_{r_{2}}+F_{\partial u, v}\left(r_{0}, r_{1}, r_{2}\right) E_{r_{0}}\left(\partial_{\mu} u\right) E_{r_{1}} v^{\mu} E_{r_{2}} \\
& \left.+g_{\mu \nu} F_{v, v}\left(r_{0}, r_{1}, r_{2}\right) E_{r_{0}} v^{\mu} E_{r_{1}} v^{\nu} E_{r_{2}}+F_{\partial v}\left(r_{0}, r_{1}\right) E_{r_{0}}\left(\partial_{\mu} v^{\mu}\right) E_{r_{1}}\right],
\end{aligned}
$$

where the sums over the $r_{0}, r_{1}, r_{2}$ in the spectrum of $u$ are omitted, the spectral functions $F$ are given below, and

$$
\begin{aligned}
\alpha:= & \frac{1}{3}\left(\partial_{\mu} \partial_{\nu} g^{\mu \nu}\right)-\frac{1}{12} g^{\mu \nu} g_{\rho \sigma}\left(\partial_{\mu} \partial_{\nu} g^{\rho \sigma}\right)+\frac{1}{48} g^{\mu \nu} g_{\rho \sigma} g_{\alpha \beta}\left(\partial_{\mu} g^{\rho \sigma}\right)\left(\partial_{\nu} g^{\alpha \beta}\right) \\
& +\frac{1}{24} g^{\mu \nu} g_{\rho \sigma} g_{\alpha \beta}\left(\partial_{\mu} g^{\rho \alpha}\right)\left(\partial_{\nu} g^{\sigma \beta}\right)-\frac{1}{12} g_{\rho \sigma}\left(\partial_{\mu} g^{\mu \nu}\right)\left(\partial_{\nu} g^{\rho \sigma}\right) \\
& +\frac{1}{12} g_{\rho \sigma}\left(\partial_{\mu} g^{\nu \rho}\right)\left(\partial_{\nu} g^{\mu \sigma}\right)-\frac{1}{4} g_{\rho \sigma}\left(\partial_{\mu} g^{\mu \rho}\right)\left(\partial_{\nu} g^{\nu \sigma}\right) .
\end{aligned}
$$

The spectral functions in (2.19) are given in terms of the universal function $I_{d / 2,1}$ by

$$
F_{w}\left(r_{0}, r_{1}\right)=I_{d / 2,1}\left(r_{0}, r_{1}\right),
$$




$$
\begin{aligned}
& F_{\partial v}\left(r_{0}, r_{1}\right)=2 r_{0} \frac{I_{d / 2,1}\left(r_{0}, r_{0}\right)-I_{d / 2,1}\left(r_{0}, r_{1}\right)}{d\left(r_{0}-r_{1}\right)} \\
& F_{\partial \partial u}\left(r_{0}, r_{1}\right)=-r_{0} \frac{4 r_{0} I_{d / 2,1}\left(r_{0}, r_{0}\right)+\left((d-4) r_{0}-d r_{1}\right) I_{d / 2,1}\left(r_{0}, r_{1}\right)}{d\left(r_{0}-r_{1}\right)^{2}}, \\
& F_{\partial u}^{\mu}\left(r_{0}, r_{1}\right)=\left[\frac{1}{2} \alpha^{\mu}-\beta^{\mu}\right] r_{0} \frac{4 r_{0} I_{d / 2,1}\left(r_{0}, r_{0}\right)+\left((d-4) r_{0}-d r_{1}\right) I_{d / 2,1}\left(r_{0}, r_{1}\right)}{d\left(r_{0}-r_{1}\right)^{2}} \\
& F_{v, \mu}\left(r_{0}, r_{1}\right)=-\alpha_{\mu} r_{0} \frac{I_{d / 2,1}\left(r_{0}, r_{0}\right)-I_{d / 2,1}\left(r_{0}, r_{1}\right)}{d\left(r_{0}-r_{1}\right)}-\frac{1}{2}\left[\frac{1}{2} \alpha_{\mu}-\beta_{\mu}\right] I_{d / 2,1}\left(r_{0}, r_{1}\right), \\
& F_{v, v}\left(r_{0}, r_{1}, r_{2}\right)=\frac{I_{d / 2,1}\left(r_{0}, r_{1}\right)-I_{d / 2,1}\left(r_{0}, r_{2}\right)}{d\left(r_{1}-r_{2}\right)} \\
& F_{\partial u, v}\left(r_{0}, r_{1}, r_{2}\right)=\frac{2 r_{0}}{d}\left[\frac{I_{d / 2,1}\left(r_{0}, r_{0}\right)}{\left(r_{0}-r_{1}\right)\left(r_{0}-r_{2}\right)}+\frac{I_{d / 2,1}\left(r_{0}, r_{1}\right)}{\left(r_{1}-r_{0}\right)\left(r_{1}-r_{2}\right)}+\frac{I_{d / 2,1}\left(r_{0}, r_{2}\right)}{\left(r_{2}-r_{0}\right)\left(r_{2}-r_{1}\right)}\right], \\
& F_{v, \partial u}\left(r_{0}, r_{1}, r_{2}\right)=-2 r_{0} \frac{I_{d / 2,1}\left(r_{0}, r_{0}\right)}{d\left(r_{0}-r_{2}\right)\left(r_{1}-r_{2}\right)}-2 r_{1} \frac{I_{d / 2,1}\left(r_{0}, r_{1}\right)}{d\left(r_{1}-r_{2}\right)^{2}} \\
& -\frac{\left((d-4) r_{0} r_{1}-(d-2) r_{0} r_{2}-(d-2) r_{1} r_{2}+d r_{2}^{2}\right) I_{d / 2,1}\left(r_{0}, r_{2}\right)}{d\left(r_{0}-r_{2}\right)\left(r_{1}-r_{2}\right)^{2}}, \\
& F_{\partial u, \partial u}\left(r_{0}, r_{1}, r_{2}\right)=\frac{4 r_{0}}{d\left(r_{0}-r_{1}\right)\left(r_{0}-r_{2}\right)^{2}\left(r_{1}-r_{2}\right)^{2}} \\
& \times\left[r_{0}\left(r_{1}-r_{2}\right)\left(r_{0}-2 r_{1}+r_{2}\right) I_{d / 2,1}\left(r_{0}, r_{0}\right)+r_{1}\left(r_{0}-r_{2}\right)^{2} I_{d / 2,1}\left(r_{0}, r_{1}\right)\right. \\
& \left.+\frac{1}{2}\left(r_{0}-r_{1}\right)\left((d-4) r_{0} r_{1}-(d-2) r_{0} r_{2}-d r_{1} r_{2}+(d+2) r_{2}^{2}\right) I_{d / 2,1}\left(r_{0}, r_{2}\right)\right] \text {. }
\end{aligned}
$$

Theorem 2.4 For $P$ given by $(2.1), a_{2}(a, P)(x)=\operatorname{tr}\left[a(x) \mathcal{R}_{2}(x)\right]$ with

$$
\begin{aligned}
\mathcal{R}_{2}=\frac{1}{2^{d} \pi^{d / 2}}[ & \frac{1}{6} R r_{0}^{-d / 2+1} E_{r_{0}}+G_{q}\left(r_{0}, r_{1}\right) E_{r_{0}} q E_{r_{1}}+g^{\mu \nu} G_{\widehat{\nabla} \widehat{\nabla} u}\left(r_{0}, r_{1}\right) E_{r_{0}}\left(\widehat{\nabla}_{\mu} \widehat{\nabla}_{\nu} u\right) E_{r_{1}} \\
& +G_{\widehat{\nabla} p}\left(r_{0}, r_{1}\right) E_{r_{0}}\left(\widehat{\nabla}_{\mu} p^{\mu}\right) E_{r_{1}}+g^{\mu \nu} G_{\widehat{\nabla} u, \widehat{\nabla} u}\left(r_{0}, r_{1}, r_{2}\right) E_{r_{0}}\left(\widehat{\nabla}_{\mu} u\right) E_{r_{1}}\left(\widehat{\nabla}_{\nu} u\right) E_{r_{2}} \\
& +G_{p, \widehat{\nabla} u}\left(r_{0}, r_{1}, r_{2}\right) E_{r_{0}} p^{\mu} E_{r_{1}}\left(\widehat{\nabla}_{\mu} u\right) E_{r_{2}}+G_{\widehat{\nabla} u, p}\left(r_{0}, r_{1}, r_{2}\right) E_{r_{0}}\left(\widehat{\nabla}_{\mu} u\right) E_{r_{1}} p^{\mu} E_{r_{2}} \\
& \left.+G_{p, p}\left(r_{0}, r_{1}, r_{2}\right) E_{r_{0}} p^{\mu} E_{r_{1}} p_{\mu} E_{r_{2}}\right]
\end{aligned}
$$

where the sums over the $r_{0}, r_{1}, r_{2}$ in the spectrum of $u$ are omitted, the spectral functions $G$ are given below, and $R$ is the scalar curvature of $g$.

The spectral functions in (2.20) are given in terms of the spectral functions $F$ by

$$
\begin{aligned}
G_{q}\left(r_{0}, r_{1}\right) & =F_{w}\left(r_{0}, r_{1}\right), \\
G_{\widehat{\nabla} \widehat{\nabla} u}\left(r_{0}, r_{1}\right) & =F_{\partial \partial u}\left(r_{0}, r_{1}\right)+F_{\partial v}\left(r_{0}, r_{1}\right), \\
G_{\widehat{\nabla} p}\left(r_{0}, r_{1}\right) & =F_{\partial v}\left(r_{0}, r_{1}\right), \\
G_{\widehat{\nabla} u, \widehat{\nabla} u}\left(r_{0}, r_{1}, r_{2}\right) & =F_{\partial u, \partial u}\left(r_{0}, r_{1}, r_{2}\right)+F_{v, \partial u}\left(r_{0}, r_{1}, r_{2}\right)+F_{\partial u, v}\left(r_{0}, r_{1}, r_{2}\right)+F_{v, v}\left(r_{0}, r_{1}, r_{2}\right) \\
G_{p, \widehat{\nabla} u}\left(r_{0}, r_{1}, r_{2}\right) & =F_{v, \partial u}\left(r_{0}, r_{1}, r_{2}\right)+F_{v, v}\left(r_{0}, r_{1}, r_{2}\right), \\
G_{\widehat{\nabla} u, p}\left(r_{0}, r_{1}, r_{2}\right) & =F_{\partial u, v}\left(r_{0}, r_{1}, r_{2}\right)+F_{v, v}\left(r_{0}, r_{1}, r_{2}\right),
\end{aligned}
$$




$$
G_{p, p}\left(r_{0}, r_{1}, r_{2}\right)=F_{v, v}\left(r_{0}, r_{1}, r_{2}\right) .
$$

As shown in [1], the universal spectral functions $I_{\alpha, k}$ are continuous, so that all the spectral functions $F$ and $G$ are also continuous, as can be deduced from their original expressions in terms of functions $I_{\alpha, k}$ given in the list (4.1) and the above relations between the $F$ and the $G$.

Remark 2.5 The metric $g$ plays a double role here: it is the metric of the Riemannian manifold $(M, g)$ and it is the non-degenerate tensor which multiply $u$ in $P$. If one has to consider two operators $P_{1}$ and $P_{2}$ with tensors $g_{1}$ and $g_{2}$ on the same manifold $M$, it may be not natural to take $g_{1}$ or $g_{2}$ as the Riemannian metric on $M$. It is possible to consider a metric $h$ on $M$ different to the tensor $g$ associated to $P$. In that case, we have to replace $\mathrm{dvol}_{g}$ in $(1.3)$ by $\mathrm{dvol}_{h}$ and $K(t, x, x$,$) is then$ a density for $h$, so that the true function is now $|h|^{-1 / 2} K(t, x, x)$, and $|h|^{-1 / 2}$ appears in (2.15) and (2.16) in place of $|g|^{-1 / 2}$. Now, the computation of $T_{k, p}$ makes apparent the coefficient $g_{d}$ given by (2.17) where the metric $g$ comes from $P$. Finally, in (2.19) and (2.20), the two determinants do not simplify anymore, and one gets an extra factor $|g|^{1 / 2}|h|^{-1 / 2}$ in front of $\mathcal{R}_{2}$, which is now relative to $\varphi_{h}(a):=\int_{M} \operatorname{tr}[a(x)] \operatorname{dvol}_{h}(x)$.

A change of connection as in Prop. 2.1 does not change the value of $\mathcal{R}_{2}$. This induces the following relations between the spectral functions $G$.

Proposition 2.6 The spectral functions $G$ satisfy the relations:

$$
\begin{aligned}
G_{\widehat{\nabla} p}\left(r_{0}, r_{1}\right) & =-\frac{r_{0} G_{q}\left(r_{0}, r_{1}\right)+\left(r_{0}-r_{1}\right) G_{\widehat{\nabla} \widehat{\nabla} u}\left(r_{0}, r_{1}\right)}{r_{0}+r_{1}}, \\
G_{\widehat{\nabla} u, p}\left(r_{0}, r_{1}, r_{2}\right) & =-\frac{r_{2} G_{q}\left(r_{0}, r_{2}\right)+\left(r_{0}+3 r_{2}\right) G_{\widehat{\nabla} \widehat{\nabla} u}\left(r_{0}, r_{2}\right)+\left(r_{0}+r_{2}\right)\left(r_{1}-r_{2}\right) G_{\widehat{\nabla} u, \widehat{\nabla} u}\left(r_{0}, r_{1}, r_{2}\right)}{\left(r_{0}+r_{2}\right)\left(r_{1}+r_{2}\right)}, \\
G_{p, \widehat{\nabla} u}\left(r_{0}, r_{1}, r_{2}\right) & =\frac{r_{0} G_{q}\left(r_{0}, r_{2}\right)+\left(3 r_{0}+r_{2}\right) G_{\widehat{\nabla} \widehat{\nabla} u}\left(r_{0}, r_{2}\right)+\left(r_{0}+r_{2}\right)\left(r_{1}-r_{0}\right) G_{\widehat{\nabla} u, \widehat{\nabla} u}\left(r_{0}, r_{1}, r_{2}\right)}{\left(r_{0}+r_{2}\right)\left(r_{1}+r_{0}\right)}, \\
G_{p, p}\left(r_{0}, r_{1}, r_{2}\right) & =-\frac{r_{1} G_{q}\left(r_{0}, r_{2}\right)-\left(r_{0}-2 r_{1}+r_{2}\right) G_{\widehat{\nabla} \widehat{\nabla} u}\left(r_{0}, r_{2}\right)+\left(r_{0}-r_{1}\right)\left(r_{1}-r_{2}\right) G_{\widehat{\nabla} u, \widehat{\nabla} u}\left(r_{0}, r_{1}, r_{2}\right)}{\left(r_{0}+r_{1}\right)\left(r_{1}+r_{2}\right)} .
\end{aligned}
$$

Proof Inserting the relations (2.6) into (2.20), all the terms involving $\phi_{\mu}$ must vanish. This induces the following relations between the $G$ functions:

$$
\begin{aligned}
& r_{0} G_{q}\left(r_{0}, r_{1}\right)+\left(r_{0}-r_{1}\right) G_{\widehat{\nabla} \widehat{\nabla} u}\left(r_{0}, r_{1}\right)+\left(r_{0}+r_{1}\right) G_{\widehat{\nabla} p}\left(r_{0}, r_{1}\right)=0, \\
& G_{\widehat{\nabla} p}\left(r_{0}, r_{2}\right)-\left(r_{0}-r_{1}\right) G_{\widehat{\nabla} u, p}\left(r_{0}, r_{1}, r_{2}\right)-\left(r_{0}+r_{1}\right) G_{p, p}\left(r_{0}, r_{1}, r_{2}\right)=0, \\
& G_{q}\left(r_{0}, r_{2}\right)+G_{\widehat{\nabla} p}\left(r_{0}, r_{2}\right)+\left(r_{1}-r_{2}\right) G_{p, \widehat{\nabla} u}\left(r_{0}, r_{1}, r_{2}\right)+\left(r_{1}+r_{2}\right) G_{p, p}\left(r_{0}, r_{1}, r_{2}\right)=0, \\
& 2 G_{\widehat{\nabla} \widehat{\nabla} u}\left(r_{0}, r_{2}\right)-G_{\widehat{\nabla} p}\left(r_{0}, r_{2}\right)-\left(r_{0}-r_{1}\right) G_{\widehat{\nabla} u, \widehat{\nabla} u}\left(r_{0}, r_{1}, r_{2}\right)-\left(r_{0}+r_{1}\right) G_{p, \widehat{\nabla} u}\left(r_{0}, r_{1}, r_{2}\right)=0, \\
& G_{q}\left(r_{0}, r_{2}\right)+2 G_{\widehat{\nabla} \widehat{\nabla} u}\left(r_{0}, r_{2}\right)+G_{\widehat{\nabla} p}\left(r_{0}, r_{2}\right) \\
&+\left(r_{1}-r_{2}\right) G_{\widehat{\nabla} u, \widehat{\nabla} u}\left(r_{0}, r_{1}, r_{2}\right)+\left(r_{1}+r_{2}\right) G_{\widehat{\nabla} u, p}\left(r_{0}, r_{1}, r_{2}\right)=0, \\
& r_{0} G_{q}\left(r_{0}, r_{2}\right)+\left(r_{0}-2 r_{1}+r_{2}\right) G_{\widehat{\nabla} \widehat{\nabla} u}\left(r_{0}, r_{2}\right)+\left(r_{0}-r_{2}\right) G_{\widehat{\nabla} p}\left(r_{0}, r_{2}\right) \\
&+\left(r_{0}-r_{1}\right)\left(r_{1}-r_{2}\right) G_{\widehat{\nabla} u, \widehat{\nabla} u}\left(r_{0}, r_{1}, r_{2}\right)+\left(r_{0}+r_{1}\right)\left(r_{1}-r_{2}\right) G_{p, \widehat{\nabla} u}\left(r_{0}, r_{1}, r_{2}\right) \\
&+\left(r_{0}-r_{1}\right)\left(r_{1}+r_{2}\right) G_{\widehat{\nabla} u, p}\left(r_{0}, r_{1}, r_{2}\right)+\left(r_{0}+r_{1}\right)\left(r_{1}+r_{2}\right) G_{p, p}\left(r_{0}, r_{1}, r_{2}\right)=0 .
\end{aligned}
$$

One can check directly that these relations hold true. From them, one can solve $G_{\widehat{\nabla} p}, G_{\widehat{\nabla} u, p}, G_{p, \widehat{\nabla} u}$, and $G_{p, p}$ in terms of $G_{q}, G_{\widehat{\nabla} \widehat{\nabla} u}$, and $G_{\widehat{\nabla} u, \widehat{\nabla} u}$. This gives the relations of the proposition. 
These relations show that the four spectral functions $G$ involved in terms with $p^{\mu}$ are deduced from the three spectral functions involving only $u$ and $q$. This result is not a surprise: from Corollary 2.2 we know that we can start with $p^{\mu}=0$, so that $\mathcal{R}_{2}$ is written in terms of the three functions $G_{q}, G_{\widehat{\nabla} \widehat{\nabla} u}, G_{\widehat{\nabla} u, \widehat{\nabla} u}$ only, and then we can change the connection in order to produce the most general expression for $\mathcal{R}_{2}$. In other words, among the seven spectral functions $G$, only three are fundamental.

The spectral functions $G$ can be computed explicitly, and their expressions depends on the value of $m$. Let

$$
\begin{aligned}
& Q_{1}(a, b, c):=\frac{-3 a^{3}+a^{2} b-6 a^{2} c+6 a b c+a c^{2}+b c^{2}}{2(a-b)^{2}(a-c)^{3}}, \\
& Q_{2}(a, b, c):=\frac{1}{2}\left[\frac{2}{(a-b)(a-c)}+\frac{(a+b)(a+c)-4 a^{2}}{(a-b)^{2}(a-c)^{2}} \ln (a)\right. \\
& \left.+\frac{a+b}{(b-a)^{2}(b-c)} \ln (b)+\frac{a+c}{(c-a)^{2}(c-b)} \ln (c)\right], \\
& Q_{3}(a, b, c):=6 \sqrt{a b c}+a^{3 / 2}+2 b^{3 / 2}+c^{3 / 2}+\sqrt{a c}(\sqrt{a}+\sqrt{c}) \\
& +2(a(\sqrt{b}+\sqrt{c})+2 b(\sqrt{a}+\sqrt{c})+c(\sqrt{a}+\sqrt{b})), \\
& Q_{4}(a, b, c):=\frac{a+b+c+2 \sqrt{a b}+2 \sqrt{a c}+\sqrt{b c}}{\sqrt{b c}(\sqrt{a}+\sqrt{b})^{2}(\sqrt{a}+\sqrt{c})^{2}(\sqrt{b}+\sqrt{c})} .
\end{aligned}
$$

Corollary 2.7 (Case $\boldsymbol{d}=2$ ) In dimension two the spectral functions $G$ can be written in terms of log functions, and this leads to:

$$
\begin{aligned}
\mathcal{R}_{2}=\frac{1}{4 \pi}[ & \frac{1}{6} R+\frac{\ln \left(r_{0}\right)-\ln \left(r_{1}\right)}{r_{0}-r_{1}} E_{r_{0}} q E_{r_{1}}+\frac{1}{r_{0}-r_{1}}\left(1-r_{0} \frac{\ln \left(r_{0}\right)-\ln \left(r_{1}\right)}{r_{0}-r_{1}}\right) E_{r_{0}}\left(\widehat{\nabla}_{\mu} p^{\mu}\right) E_{r_{1}} \\
& -\frac{1}{\left(r_{0}-r_{1}\right)^{2}}\left(r_{0}+r_{1}-2 r_{0} r_{1} \frac{\ln \left(r_{0}\right)-\ln \left(r_{1}\right)}{r_{0}-r_{1}}\right) g^{\mu \nu} E_{r_{0}}\left(\widehat{\nabla}_{\mu} \widehat{\nabla}_{\nu} u\right) E_{r_{1}} \\
+ & \left(\frac{\left(r_{0}+r_{2}\right)\left(r_{0}-2 r_{1}+r_{2}\right)}{\left(r_{0}-r_{1}\right)\left(r_{0}-r_{2}\right)^{2}\left(r_{1}-r_{2}\right)}-Q_{1}\left(r_{0}, r_{1}, r_{2}\right) \ln \left(r_{0}\right)\right. \\
& \left.\quad-\frac{\left(r_{0}+r_{1}\right)\left(r_{1}+r_{2}\right)}{2\left(r_{0}-r_{1}\right)^{2}\left(r_{1}-r_{2}\right)^{2}} \ln \left(r_{1}\right)-Q_{1}\left(r_{2}, r_{1}, r_{0}\right) \ln \left(r_{2}\right)\right) g^{\mu \nu} E_{r_{0}}\left(\widehat{\nabla}_{\mu} u\right) E_{r_{1}}\left(\widehat{\nabla}_{\nu} u\right) E_{r_{2}} \\
+ & Q_{2}\left(r_{0}, r_{1}, r_{2}\right) E_{r_{0}}\left(\widehat{\nabla}_{\mu} u\right) E_{r_{1}} p^{\mu} E_{r_{2}}-Q_{2}\left(r_{2}, r_{1}, r_{0}\right) E_{r_{0}} p^{\mu} E_{r_{1}}\left(\widehat{\nabla}_{\mu} u\right) E_{r_{2}} \\
+ & \left.\frac{1}{2}\left(\frac{\ln \left(r_{0}\right)}{\left(r_{0}-r_{1}\right)\left(r_{0}-r_{2}\right)}+\frac{\ln \left(r_{1}\right)}{\left(r_{1}-r_{0}\right)\left(r_{1}-r_{2}\right)}+\frac{\ln \left(r_{2}\right)}{\left(r_{2}-r_{0}\right)\left(r_{2}-r_{1}\right)}\right) E_{r_{0}} p_{\mu} E_{r_{1}} p^{\mu} E_{r_{2}}\right] .
\end{aligned}
$$

When $d=2 m \geq 4$ is even, all the functions involved are Laurent polynomials. This is a direct consequence of [1, Prop. 3.5]:

$$
\begin{aligned}
G_{q}\left(r_{0}, r_{1}\right) & =\sum_{0 \leq \ell \leq m-2} \frac{1}{m-1} r_{0}^{\ell+1-m} r_{1}^{-\ell-1}, \\
G_{\widehat{\nabla} \widehat{\nabla} u}\left(r_{0}, r_{1}\right) & =-\sum_{0 \leq \ell \leq m-2} \frac{(m-\ell-1)(\ell+1)}{m(m-1)} r_{0}^{\ell+1-m} r_{1}^{-\ell-1},
\end{aligned}
$$




$$
\begin{aligned}
G_{\widehat{\nabla} p}\left(r_{0}, r_{1}\right) & =-\sum_{0 \leq \ell \leq m-2} \frac{m-\ell-1}{m(m-1)} r_{0}^{\ell+1-m} r_{1}^{-\ell-1}, \\
G_{\widehat{\nabla} u, \widehat{\nabla} u}\left(r_{0}, r_{1}, r_{2}\right) & =-\sum_{0 \leq \ell \leq k \leq m-2} \frac{(2 \ell+1)(2 k-2 m+3)}{2 m(m-1)} r_{0}^{k+1-m} r_{1}^{\ell-k-1} r_{2}^{-\ell-1}, \\
G_{p, \widehat{\nabla} u}\left(r_{0}, r_{1}, r_{2}\right) & =-\sum_{0 \leq \ell \leq k \leq m-2} \frac{2 \ell+1}{2 m(m-1)} r_{0}^{k+1-m} r_{1}^{\ell-k-1} r_{2}^{-\ell-1}, \\
G_{\widehat{\nabla} u, p}\left(r_{0}, r_{1}, r_{2}\right) & =-\sum_{0 \leq \ell \leq k \leq m-2} \frac{2 k-2 m+3}{2 m(m-1)} r_{0}^{k+1-m} r_{1}^{\ell-k-1} r_{2}^{-\ell-1}, \\
G_{p, p}\left(r_{0}, r_{1}, r_{2}\right) & =-\sum_{0 \leq \ell \leq k \leq m-2} \frac{1}{2 m(m-1)} r_{0}^{k+1-m} r_{1}^{\ell-k-1} r_{2}^{-\ell-1} .
\end{aligned}
$$

This implies the following expressions for $\mathcal{R}_{2}$ :

Corollary 2.8 (Case $\boldsymbol{d}=\mathbf{2 m}$ even and $\boldsymbol{d} \geq 4$ ) Using the expressions of the spectral functions $G$ as Laurent polynomials, one has

$$
\begin{aligned}
\mathcal{R}_{2}=\frac{1}{2^{2 m} \pi^{m}}[ & \frac{1}{6} R u^{-m+1}+\sum_{0 \leq \ell \leq m-2} \frac{1}{m-1} u^{\ell+1-m} q u^{-\ell-1}-\sum_{0 \leq \ell \leq m-2} \frac{m-\ell-1}{m(m-1)} u^{\ell+1-m}\left(\widehat{\nabla}_{\mu} p^{\mu}\right) u^{-\ell-1} \\
& -\sum_{0 \leq \ell \leq m-2} \frac{(m-\ell-1)(\ell+1)}{m(m-1)} g^{\mu \nu} u^{\ell+1-m}\left(\widehat{\nabla}_{\mu} \widehat{\nabla}_{\nu} u\right) u^{-\ell-1} \\
& -\sum_{0 \leq \ell \leq k \leq m-2} \frac{(2 \ell+1)(2 k-2 m+3)}{2 m(m-1)} g^{\mu \nu} u^{k+1-m}\left(\widehat{\nabla}_{\mu} u\right) u^{\ell-k-1}\left(\widehat{\nabla}_{\nu} u\right) u^{-\ell-1} \\
& -\sum_{0 \leq \ell \leq k \leq m-2} \frac{2 k-2 m+3}{2 m(m-1)} u^{k+1-m}\left(\widehat{\nabla}_{\mu} u\right) u^{\ell-k-1} p^{\mu} u^{-\ell-1} \\
& -\sum_{0 \leq \ell \leq k \leq m-2} \frac{2 \ell+1}{2 m(m-1)} u^{k+1-m} p^{\mu} u^{\ell-k-1}\left(\widehat{\nabla}_{\mu} u\right) u^{-\ell-1} \\
& \left.-\sum_{0 \leq \ell \leq k \leq m-2} \frac{1}{2 m(m-1)} u^{k+1-m} p_{\mu} u^{\ell-k-1} p^{\mu} u^{-\ell-1}\right] \\
\frac{1}{2^{2 m} \pi^{m}} & {\left[\frac{1}{6} R u^{-m+1}+\sum_{0 \leq \ell \leq m-2} \frac{1}{m-1} u^{\ell+1-m} q u^{-\ell-1}\right.} \\
& -\sum_{0 \leq \ell \leq m-2} \frac{(m-\ell-1)}{m(m-1)} g^{\mu \nu} u^{\ell+1-m}\left[\widehat{\nabla}_{\mu}\left((\ell+1) \widehat{\nabla}_{\nu} u+p_{\nu}\right)\right] u^{-\ell-1} \\
& \left.-\sum_{0 \leq \ell \leq k \leq m-2} \frac{1}{2 m(m-1)} g^{\mu \nu} u^{k+1-m}\left[(2 k-2 m+3) \widehat{\nabla}_{\mu} u+p_{\mu}\right] u^{\ell-k-1}\left[(2 \ell+1) \widehat{\nabla}_{\nu} u+p_{\nu}\right] u^{-\ell-1}\right] .
\end{aligned}
$$

Corollary 2.9 (Case $\boldsymbol{d}=4$ ) In dimension four these expressions simplify further to:

$$
\begin{aligned}
\mathcal{R}_{2}=\frac{1}{16 \pi^{2}}[ & \frac{1}{6} R u^{-1}+u^{-1} q u^{-1}-\frac{1}{2} u^{-1}\left(\widehat{\nabla}_{\mu} p^{\mu}\right) u^{-1}-\frac{1}{2} g^{\mu \nu} u^{-1}\left(\widehat{\nabla}_{\mu} \widehat{\nabla}_{\nu} u\right) u^{-1} \\
& +\frac{1}{4} g^{\mu \nu} u^{-1}\left(\widehat{\nabla}_{\mu} u\right) u^{-1}\left(\widehat{\nabla}_{\nu} u\right) u^{-1}+\frac{1}{4} u^{-1}\left(\widehat{\nabla}_{\mu} u\right) u^{-1} p^{\mu} u^{-1}-\frac{1}{4} u^{-1} p^{\mu} u^{-1}\left(\widehat{\nabla}_{\mu} u\right) u^{-1} \\
& \left.\quad-\frac{1}{4} u^{-1} p_{\mu} u^{-1} p^{\mu} u^{-1}\right] \\
=\frac{1}{16 \pi^{2}}[ & \left.\frac{1}{6} R u^{-1}+u^{-1} q u^{-1}-\frac{1}{2} g^{\mu \nu} u^{-1}\left[\widehat{\nabla}_{\mu}\left(\widehat{\nabla}_{\nu} u+p_{\nu}\right)\right] u^{-1}+\frac{1}{4} g^{\mu \nu} u^{-1}\left[\widehat{\nabla}_{\mu} u-p_{\mu}\right] u^{-1}\left[\widehat{\nabla}_{\nu} u+p_{\nu}\right] u^{-1}\right] .
\end{aligned}
$$


Corollary 2.10 (Case $\boldsymbol{d}=3$ ) In dimension three the spectral functions $G$ can be written in terms of square roots of the $r_{i}$ (see [1, Prop. 3.4]), and this leads to:

$$
\begin{aligned}
\mathcal{R}_{2}=\frac{1}{8 \pi^{3 / 2}}[ & \frac{1}{6} R+\frac{2}{\sqrt{r_{0} r_{1}}\left(\sqrt{r_{0}}+\sqrt{r_{1}}\right)} E_{r_{0}} q E_{r_{1}}-\frac{2}{3} \frac{2 \sqrt{r_{0}}+\sqrt{r_{1}}}{\sqrt{r_{0} r_{1}}\left(\sqrt{r_{0}}+\sqrt{r_{1}}\right)^{2}} E_{r_{0}}\left(\widehat{\nabla}_{\mu} p^{\mu}\right) E_{r_{1}} \\
& -\frac{2}{3} \frac{\sqrt{r_{0} r_{1}}+\left(\sqrt{r_{0}}+\sqrt{r_{1}}\right)^{2}}{\sqrt{r_{0} r_{1}}\left(\sqrt{r_{0}}+\sqrt{r_{1}}\right)^{3}} g^{\mu \nu} E_{r_{0}}\left(\widehat{\nabla}, \widehat{\nabla}_{\nu} u\right) E_{r_{1}} \\
& +\frac{2}{3} \frac{Q_{3}\left(r_{0}, r_{1}, r_{2}\right)}{\sqrt{r_{1}}\left(\sqrt{r_{0}}+\sqrt{r_{1}}\right)^{2}\left(\sqrt{r_{0}}+\sqrt{r_{2}}\right)^{3}\left(\sqrt{r_{1}}+\sqrt{r_{2}}\right)^{2}} g^{\mu \nu} E_{r_{0}}\left(\widehat{\nabla}_{\mu} u\right) E_{r_{1}}\left(\widehat{\nabla}_{\nu} u\right) E_{r_{2}} \\
& +\frac{2}{3} Q_{4}\left(r_{0}, r_{1}, r_{2}\right) E_{r_{0}}(\widehat{\nabla} \mu u) E_{r_{1}} p^{\mu} E_{r_{2}}-\frac{2}{3} Q_{4}\left(r_{2}, r_{1}, r_{0}\right) E_{r_{0}} p_{\mu} E_{r_{1}} p^{\mu} E_{r_{2}} \\
& \left.-\frac{2}{3} \frac{\sqrt{r_{0}}+\sqrt{r_{1}}+\sqrt{r_{2}}}{\sqrt{r_{0} r_{1} r_{2}}\left(\sqrt{r_{0}}+\sqrt{r_{1}}\right)\left(\sqrt{r_{0}}+\sqrt{r_{2}}\right)\left(\sqrt{r_{1}}+\sqrt{r_{2}}\right)} E_{r_{0}} p_{\mu} E_{r_{1}} p^{\mu} E_{r_{2}}\right] .
\end{aligned}
$$

To conclude this list of results given at various dimensions, we see that we have explicit expressions of $\mathcal{R}_{2}$ for $d$ even, and moreover simple generic expressions for $d=2 m, d \geq 4$, while for $d$ odd it is difficult to propose a generic expression.

\section{Some direct applications}

\subsection{The case $a=\mathbb{1}$}

When $a=\mathbb{1}$, a cyclic operation can be performed under the trace:

$$
\begin{aligned}
f\left(r_{0}, r_{1}, \ldots, r_{k}\right) \operatorname{tr}\left(E_{r_{0}} B_{1} E_{r_{1}} B_{2} \cdots B_{k} E_{r_{k}}\right) & =f\left(r_{0}, r_{1}, \ldots, r_{k}\right) \operatorname{tr}\left(E_{r_{k}} E_{r_{0}} B_{1} E_{r_{1}} B_{2} \cdots B_{k}\right) \\
& =\delta_{r_{0}, r_{k}} f\left(r_{0}, r_{1}, \ldots, r_{k}\right) \operatorname{tr}\left(E_{r_{k}} E_{r_{0}} B_{1} E_{r_{1}} B_{2} \cdots B_{k}\right) \\
& =f\left(r_{0}, r_{1}, \ldots, r_{0}\right) \operatorname{tr}\left(E_{r_{0}} B_{1} E_{r_{1}} B_{2} \cdots E_{r_{k-1}} B_{k}\right) .
\end{aligned}
$$

This implies that in all the spectral functions one can put $r_{k}=r_{0}$ (remember that all the spectral functions are continuous, so that $r_{k} \rightarrow r_{0}$ is well-defined).

In [1, Theorem 4.3], $a_{2}(\mathbb{1}, P)$ has been computed for $d=2 m$ even, $m \geq 1$. Let us first rewrite this result in terms of spectral functions:

$$
\begin{aligned}
& \alpha r_{0}^{-m+1} E_{0}+r_{0}^{-m} E_{0} w+\frac{m-2}{6}\left[\frac{1}{2} \alpha^{\mu}-\beta^{\mu}\right] r_{0}^{-m} E_{0}\left(\partial_{\mu} u\right)-\frac{m-2}{6} g^{\mu \nu} r_{0}^{-m} E_{0}\left(\partial_{\mu} \partial_{\nu} u\right) \\
& +\frac{1}{2} \beta_{\mu} r_{0}^{-m} E_{0} v^{\mu}-\frac{1}{2} r_{0}^{-m} E_{0}\left(\partial_{\mu} v^{\mu}\right)-\frac{1}{4 m} g_{\mu \nu} \sum_{\ell=0}^{m-1} r_{0}^{-\ell-1} r_{1}^{\ell-m} E_{0} v^{\mu} E_{1} v^{\nu} \\
& +\frac{1}{2 m} \sum_{\ell=0}^{m-1}(m-2 \ell) r_{0}^{-\ell-1} r_{1}^{\ell-m} E_{0} v^{\mu} E_{1}\left(\partial_{\mu} u\right)+g^{\mu \nu} \sum_{\ell=0}^{m-1}\left[\frac{m-2}{6}-\frac{\ell(m-\ell-1)}{2 m}\right] r_{0}^{-\ell-1} r_{1}^{\ell-m} E_{0}\left(\partial_{\mu} u\right) E_{1}\left(\partial_{\nu} u\right) .
\end{aligned}
$$

Notice that:

$g_{\mu \nu} \sum_{\ell=0}^{m-1} r_{0}^{-\ell-1} r_{1}^{\ell-m} \operatorname{tr}\left(E_{0} v^{\mu} E_{1} v^{\nu}\right)=g_{\mu \nu} \sum_{\ell=0}^{m-1} r_{0}^{-\ell-1} r_{1}^{\ell-m} \operatorname{tr}\left(E_{1} v^{\nu} E_{0} v^{\mu}\right)=g_{\mu \nu} \sum_{\ell=0}^{m-1} r_{1}^{-\ell-1} r_{0}^{\ell-m} \operatorname{tr}\left(E_{1} v^{\mu} E_{0} v^{\nu}\right)$

if we change $\ell$ to $m-\ell-1$ in the summation and we use the symmetry of $g_{\mu \nu}$. Then, to show that the spectral functions $F$ reduce to the ones above, one has to use the symmetry $r_{0} \leftrightarrow r_{1}$ for some terms. A direct computation gives

$$
F_{w}\left(r_{0}, r_{0}\right)=r_{0}^{-m}
$$




$$
\begin{aligned}
F_{\partial u}^{\mu}\left(r_{0}, r_{0}\right) & =\frac{m-2}{6}\left[\frac{1}{2} \alpha^{\mu}-\beta^{\mu}\right] r_{0}^{-m}, \\
F_{\partial \partial u}\left(r_{0}, r_{0}\right) & =-\frac{m-2}{6} r_{0}^{-m}, \\
F_{v, \mu}\left(r_{0}, r_{0}\right) & =\frac{1}{2} \beta_{\mu} r_{0}^{-m}, \\
F_{\partial v}\left(r_{0}, r_{0}\right) & =-\frac{1}{2} r_{0}^{-m}, \\
\frac{1}{2}\left[F_{v, v}\left(r_{0}, r_{1}, r_{0}\right)+F_{v, v}\left(r_{1}, r_{0}, r_{1}\right)\right] & =-\frac{1}{4 m} \sum_{\ell=0}^{m-1} r_{0}^{-\ell-1} r_{1}^{\ell-m}, \\
\frac{1}{2}\left[F_{\partial u, \partial u}\left(r_{0}, r_{1}, r_{0}\right)+F_{\partial u, \partial u}\left(r_{1}, r_{0}, r_{1}\right)\right] & =\sum_{\ell=0}^{m-1}\left[\frac{m-2}{6}-\frac{\ell(m-\ell-1)}{2 m}\right] r_{0}^{-\ell-1} r_{1}^{\ell-m}, \\
F_{v, \partial u}\left(r_{0}, r_{1}, r_{0}\right)+F_{\partial u, v}\left(r_{1}, r_{0}, r_{1}\right) & =\frac{1}{2 m} \sum_{\ell=0}^{m-1}(m-2 \ell) r_{0}^{-\ell-1} r_{1}^{\ell-m} .
\end{aligned}
$$

In the last expression, under the trace we have $\operatorname{tr}\left(E_{0}\left(\partial_{\mu} u\right) E_{1} v^{\mu}\right)=\operatorname{tr}\left(E_{1} v^{\mu} E_{0}\left(\partial_{\mu} u\right)\right)$, and we need to sum the two functions $F_{v, \partial u}$ and $F_{\partial u, v}$ with their correct arguments.

These relations show that (2.19) reproduces [1, eq. 4.15] when $a=\mathbb{1}$.

\subsection{Minimal Laplace type operators: $u=\mathbb{1}$}

Starting from (2.1) when $u=\mathbb{1}$ one gets

$$
P=-g^{\mu \nu} \nabla_{\mu} \nabla_{\nu}-\left(p^{\nu}-\left[\frac{1}{2} \alpha^{\nu}-\beta^{\nu}\right]\right) \nabla_{\nu}-q
$$

and two simplifications occur in (2.20): all derivatives of $u$ vanish, and the spectrum of $u$ reduces to $\operatorname{sp}(u)=\{1\}$, so that all spectral functions are taken at $r_{i}=1$ and $E_{r_{i}}=\mathbb{1}$. The result is then

$$
\mathcal{R}_{2}=\frac{1}{2^{d} \pi^{d / 2}}\left[\frac{1}{6} R+G_{q}(1,1) q+G_{\widehat{\nabla} p}(1,1) \widehat{\nabla}_{\mu} p^{\mu}+G_{p, p}(1,1,1) p^{\mu} p_{\mu}\right] .
$$

A direct computation gives $G_{q}(1,1)=1, G_{\widehat{\nabla} p}(1,1)=-\frac{1}{2}$, and $G_{p, p}(1,1,1)=-\frac{1}{4}$, so that

$$
\mathcal{R}_{2}=\frac{1}{2^{d} \pi^{d / 2}}\left[\frac{1}{6} R+q-\frac{1}{2} \widehat{\nabla}_{\mu} p^{\mu}-\frac{1}{4} p^{\mu} p_{\mu}\right] .
$$

As in Prop. 2.1, we can change $\widehat{\nabla}_{\mu}$ to $\widehat{\nabla}_{\mu}^{\prime}:=\widehat{\nabla}_{\mu}+\phi_{\mu}$ and solve $\phi_{\mu}$ in order to get $p^{\mu \prime}=0$. From (2.4) with $u=\mathbb{1}$, one has

$$
\begin{aligned}
g^{\mu \nu} \widehat{\nabla}_{\mu}^{\prime} \widehat{\nabla}_{\nu}^{\prime}+q^{\prime} & =g^{\mu \nu}\left(\widehat{\nabla}_{\mu}+\phi_{\mu}\right)\left(\widehat{\nabla}_{\nu}+\phi_{\nu}\right)+q^{\prime}=g^{\mu \nu} \widehat{\nabla}_{\mu} \widehat{\nabla}_{\nu}+g^{\mu \nu}\left(\widehat{\nabla}_{\mu} \phi_{\nu}\right)+2 g^{\mu \nu} \phi_{\nu} \widehat{\nabla}_{\mu}+g^{\mu \nu} \phi_{\mu} \phi_{\nu}+q^{\prime} \\
& =: g^{\mu \nu} \widehat{\nabla}_{\mu} \widehat{\nabla}_{\nu}+p^{\mu} \widehat{\nabla}_{\mu}+q
\end{aligned}
$$

with $p^{\mu}=2 g^{\mu \nu} \phi_{\nu}$ and $q=q^{\prime}+g^{\mu \nu}\left(\widehat{\nabla}_{\mu} \phi_{\nu}\right)+g^{\mu \nu} \phi_{\mu} \phi_{\nu}$. This is solved for $\phi_{\mu}=\frac{1}{2} g_{\mu \nu} p^{\nu}$ and implies $q^{\prime}=q-\frac{1}{2} \widehat{\nabla}_{\mu} p^{\mu}-\frac{1}{4} p^{\mu} p_{\mu}$. Injected into (3.1), this gives $\mathcal{R}_{2}=\frac{1}{2^{d} \pi^{d / 2}}\left[\frac{1}{6} R+q^{\prime}\right]$ as in $[23$, Theorem 3.3.1].

\subsection{Conformal like transformed Laplacian}

Let us consider a positive invertible element $k \in \Gamma(\operatorname{End}(V))$, a covariant derivative $\nabla_{\mu}$ on $V$ and $\Delta=-g^{\mu \nu} \widehat{\nabla}_{\mu} \widehat{\nabla}_{\nu}$ be its associated Laplacian. We consider the operator

$$
P:=k \Delta k=-g^{\mu \nu} k^{2} \widehat{\nabla}_{\mu} \widehat{\nabla}_{\nu}-2 g^{\mu \nu} k\left(\widehat{\nabla}_{\mu} k\right) \widehat{\nabla}_{\nu}+k(\Delta k) .
$$

It can be written as in (2.4) with

$$
u=k^{2}, \quad p^{\nu}=g^{\mu \nu} k\left(\widehat{\nabla}_{\mu} k\right)-g^{\mu \nu}\left(\widehat{\nabla}_{\mu} k\right) k, \quad q=-k(\Delta k) .
$$


Application of Theorem 2.4 gives

$$
\begin{aligned}
\mathcal{R}_{2}^{k \Delta k}=\frac{1}{2^{d} \pi^{d / 2}}\left[\frac{1}{6} R\right. & r_{0}^{-d / 2+1} E_{r_{0}} \\
& \left.+F_{\Delta k}^{k \Delta k}\left(r_{0}, r_{1}\right) E_{r_{0}}(\Delta k) E_{r_{1}}+F_{\nabla k \nabla k}^{k \Delta k}\left(r_{0}, r_{1}, r_{2}\right) g^{\mu \nu} E_{r_{0}}\left(\nabla_{\mu} k\right) E_{r_{1}}\left(\nabla_{\nu} k\right) E_{r_{2}}\right]
\end{aligned}
$$

with

$$
\begin{aligned}
F_{\Delta k}^{k \Delta k}\left(r_{0}, r_{1}\right)= & -\sqrt{r_{0}} G_{q}\left(r_{0}, r_{1}\right)-\left(\sqrt{r_{0}}+\sqrt{r_{1}}\right) G_{\widehat{\nabla} \widehat{\nabla} u}\left(r_{0}, r_{1}\right)-\left(\sqrt{r_{0}}-\sqrt{r_{1}}\right) G_{\widehat{\nabla} p}\left(r_{0}, r_{1}\right), \\
F_{\nabla k \nabla k}^{k \Delta k}\left(r_{0}, r_{1}, r_{2}\right)= & 2 G_{\widehat{\nabla} \widehat{\nabla} u}\left(r_{0}, r_{2}\right) \\
& +\left(\sqrt{r_{0}}+\sqrt{r_{1}}\right)\left(\sqrt{r_{1}}+\sqrt{r_{2}}\right) G_{\widehat{\nabla} u, \widehat{\nabla} u}\left(r_{0}, r_{1}, r_{2}\right) \\
& +\left(\sqrt{r_{0}}-\sqrt{r_{1}}\right)\left(\sqrt{r_{1}}+\sqrt{r_{2}}\right) G_{p, \widehat{\nabla} u}\left(r_{0}, r_{1}, r_{2}\right) \\
& +\left(\sqrt{r_{0}}+\sqrt{r_{1}}\right)\left(\sqrt{r_{1}}-\sqrt{r_{2}}\right) G_{\widehat{\nabla} u, p}\left(r_{0}, r_{1}, r_{2}\right) \\
& +\left(\sqrt{r_{0}}-\sqrt{r_{1}}\right)\left(\sqrt{r_{1}}-\sqrt{r_{2}}\right) G_{p, p}\left(r_{0}, r_{1}, r_{2}\right) .
\end{aligned}
$$

Using Proposition 2.6, one has

$$
F_{\Delta k}^{k \Delta k}\left(r_{0}, r_{1}\right)=-\frac{\sqrt{r_{0} r_{1}}\left(\sqrt{r_{0}}+\sqrt{r_{1}}\right)\left[G_{q}\left(r_{0}, r_{1}\right)+2 G_{\widehat{\nabla} \widehat{\nabla} u}\left(r_{0}, r_{1}\right)\right]}{r_{0}+r_{1}} .
$$

\section{Details of the computations}

In this section we give some details on the computations to establish Theorems 2.3 and 2.4. These computations can be done by hand but the reader can also follow [5].

The equation of (2.19) requires to compute the terms in the sum (2.16), which itself requires to compute the arguments $B_{1} \otimes \cdots \otimes B_{k}$ and the operators $T_{k, p}$.

For $r=2$, the list of arguments has been evaluated in [1, Section 4.1] (starting with the expression (1.1) of $P$ ) as well as their contractions with the tensor $G(g)_{\mu_{1} \ldots \mu_{2 p}}$. We make use of these results below. Then the computation of the operators $T_{k, p}$ reduces to the computation of the universal spectral functions $I_{d / 2+p, k}$. As noticed in [1], only the values $k=1,2,3,4$ have to be considered.

Below is the list of the evaluation of these arguments in the corresponding operators $T_{k, p}$, where the following functional calculus rule (and its obvious generalizations) is used

$$
f\left(r_{0}, r_{1}, \ldots, r_{k}\right) E_{r_{0}} u^{n_{0}} E_{r_{1}} u^{n_{1}} E_{r_{2}} \cdots u^{n_{k-1}} E_{r_{k}}=r_{0}^{n_{0}+n_{1}+\cdots+n_{k-1}} f\left(r_{0}, r_{0}, \ldots, r_{0}\right) E_{r_{0}},
$$

where summations over $r_{i}$ in the spectrum of $u$ are omitted. In the following, the symbol $\rightsquigarrow$ is used to symbolize this evaluation.

For $k=1$, there is only one argument:

$$
w \rightsquigarrow I_{d / 2,1}\left(r_{0}, r_{1}\right) E_{r_{0}} w E_{r_{1}}
$$

For $k=2$, one has:

$$
\begin{aligned}
-\frac{1}{2} g^{\mu \nu} g_{\rho \sigma}\left(\partial_{\mu} \partial_{\nu} g^{\rho \sigma}\right) u \otimes u & \rightsquigarrow-\frac{1}{2} g^{\mu \nu} g_{\rho \sigma}\left(\partial_{\mu} \partial_{\nu} g^{\rho \sigma}\right) r_{0}^{2} I_{d / 2+1,2}\left(r_{0}, r_{0}, r_{0}\right) E_{r_{0}}, \\
-g^{\mu \nu} g_{\rho \sigma}\left(\partial_{\nu} g^{\rho \sigma}\right) u \otimes \partial_{\mu} u & \rightsquigarrow-g^{\mu \nu} g_{\rho \sigma}\left(\partial_{\nu} g^{\rho \sigma}\right) r_{0} I_{d / 2+1,2}\left(r_{0}, r_{0}, r_{1}\right) E_{r_{0}}\left(\partial_{\mu} u\right) E_{r_{1}}, \\
-\frac{d}{2} g^{\mu \nu} u \otimes \partial_{\mu} \partial_{\nu} u & \rightsquigarrow-\frac{d}{2} g^{\mu \nu} r_{0} I_{d / 2+1,2}\left(r_{0}, r_{0}, r_{1}\right) E_{r_{0}}\left(\partial_{\mu} \partial_{\nu} u\right) E_{r_{1}}, \\
-\frac{1}{2} g_{\rho \sigma}\left(\partial_{\mu} g^{\rho \sigma}\right) v^{\mu} \otimes u & \rightsquigarrow-\frac{1}{2} g_{\rho \sigma}\left(\partial_{\mu} g^{\rho \sigma}\right) r_{1} I_{d / 2+1,2}\left(r_{0}, r_{1}, r_{1}\right) E_{r_{0}} v^{\mu} E_{r_{1}},
\end{aligned}
$$




$$
\begin{gathered}
-\frac{d}{2} v^{\mu} \otimes \partial_{\mu} u \rightsquigarrow-\frac{d}{2} I_{d / 2+1,2}\left(r_{0}, r_{1}, r_{2}\right) E_{r_{0}} v^{\mu} E_{r_{1}}\left(\partial_{\mu} u\right) E_{r_{2}}, \\
-\frac{1}{2} g_{\mu \nu} v^{\mu} \otimes v^{\nu} \rightsquigarrow-\frac{1}{2} g_{\mu \nu} I_{d / 2+1,2}\left(r_{0}, r_{1}, r_{2}\right) E_{r_{0}} v^{\mu} E_{r_{1}} v^{\nu} E_{r_{2}}, \\
-u \otimes \partial_{\mu} v^{\mu} \rightsquigarrow-r_{0} I_{d / 2+1,2}\left(r_{0}, r_{0}, r_{1}\right) E_{r_{0}}\left(\partial_{\mu} v^{\mu}\right) E_{r_{1}} .
\end{gathered}
$$

For $k=3$, one has:

$$
\begin{aligned}
& {\left[g^{\mu \nu} g_{\rho \sigma}\left(\partial_{\mu} \partial_{\nu} g^{\rho \sigma}\right)+2\left(\partial_{\mu} \partial_{\nu} g^{\mu \nu}\right)+g_{\rho \sigma}\left(\partial_{\mu} g^{\mu \nu}\right)\left(\partial_{\nu} g^{\rho \sigma}\right)+2 g_{\rho \sigma}\left(\partial_{\mu} g^{\nu \rho}\right)\left(\partial_{\nu} g^{\mu \sigma}\right)\right.} \\
& \left.+\frac{1}{2} g^{\mu \nu} g_{\rho \sigma} g_{\alpha \beta}\left(\partial_{\mu} g^{\rho \sigma}\right)\left(\partial_{\nu} g^{\alpha \beta}\right)+g^{\mu \nu} g_{\rho \sigma} g_{\alpha \beta}\left(\partial_{\mu} g^{\rho \alpha}\right)\left(\partial_{\nu} g^{\sigma \beta}\right)\right] u \otimes u \otimes u \\
& \rightsquigarrow \\
& \quad\left[g^{\mu \nu} g_{\rho \sigma}\left(\partial_{\mu} \partial_{\nu} g^{\rho \sigma}\right)+2\left(\partial_{\mu} \partial_{\nu} g^{\mu \nu}\right)+g_{\rho \sigma}\left(\partial_{\mu} g^{\mu \nu}\right)\left(\partial_{\nu} g^{\rho \sigma}\right)+2 g_{\rho \sigma}\left(\partial_{\mu} g^{\nu \rho}\right)\left(\partial_{\nu} g^{\mu \sigma}\right)\right. \\
& \left.\quad+\frac{1}{2} g^{\mu \nu} g_{\rho \sigma} g_{\alpha \beta}\left(\partial_{\mu} g^{\rho \sigma}\right)\left(\partial_{\nu} g^{\alpha \beta}\right)+g^{\mu \nu} g_{\rho \sigma} g_{\alpha \beta}\left(\partial_{\mu} g^{\rho \alpha}\right)\left(\partial_{\nu} g^{\sigma \beta}\right)\right] r_{0}^{3} I_{d / 2+2,3}\left(r_{0}, r_{0}, r_{0}, r_{0}\right) E_{r_{0}}, \\
& (d+6)\left[\frac{1}{2} g^{\mu \nu} g_{\rho \sigma}\left(\partial_{\nu} g^{\rho \sigma}\right)+\left(\partial_{\nu} g^{\mu \nu}\right)\right] u \otimes u \otimes \partial_{\mu} u \\
& \rightsquigarrow(d+6)\left[\frac{1}{2} g^{\mu \nu} g_{\rho \sigma}\left(\partial_{\nu} g^{\rho \sigma}\right)+\left(\partial_{\nu} g^{\mu \nu}\right)\right] r_{0}^{2} I_{d / 2+2,3}\left(r_{0}, r_{0}, r_{0}, r_{1}\right) E_{r_{0}}\left(\partial_{\mu} u\right) E_{r_{1}}
\end{aligned}
$$$$
\rightsquigarrow\left[\frac{d+4}{2} g^{\mu \nu} g_{\rho \sigma}\left(\partial_{\nu} g^{\rho \sigma}\right)+2\left(\partial_{\nu} g^{\mu \nu}\right)\right] r_{0} r_{1} I_{d / 2+2,3}\left(r_{0}, r_{0}, r_{1}, r_{1}\right) E_{r_{0}}\left(\partial_{\mu} u\right) E_{r_{1}},
$$$$
\frac{(d+2)^{2}}{2} g^{\mu \nu} u \otimes \partial_{\mu} u \otimes \partial_{\nu} u \rightsquigarrow \frac{(d+2)^{2}}{2} g^{\mu \nu} r_{0} I_{d / 2+2,3}\left(r_{0}, r_{0}, r_{1}, r_{2}\right) E_{r_{0}}\left(\partial_{\mu} u\right) E_{r_{1}}\left(\partial_{\nu} u\right) E_{r_{2}} \text {, }
$$$$
(d+2) g^{\mu \nu} u \otimes u \otimes \partial_{\mu} \partial_{\nu} u \rightsquigarrow(d+2) g^{\mu \nu} r_{0}^{2} I_{d / 2+2,3}\left(r_{0}, r_{0}, r_{0}, r_{1}\right) E_{r_{0}}\left(\partial_{\mu} \partial_{\nu} u\right) E_{r_{1}} \text {, }
$$$$
\left[\frac{1}{2} g_{\rho \sigma}\left(\partial_{\mu} g^{\rho \sigma}\right)+g_{\mu \nu}\left(\partial_{\rho} g^{\rho \nu}\right)\right]\left(v^{\mu} \otimes u \otimes u+u \otimes v^{\mu} \otimes u+u \otimes u \otimes v^{\mu}\right)
$$$$
\rightsquigarrow\left[\frac{1}{2} g_{\rho \sigma}\left(\partial_{\mu} g^{\rho \sigma}\right)+g_{\mu \nu}\left(\partial_{\rho} g^{\rho \nu}\right)\right]\left[r_{1}^{2} I_{d / 2+2,3}\left(r_{0}, r_{1}, r_{1}, r_{1}\right)\right.
$$$$
\left.+r_{0} r_{1} I_{d / 2+2,3}\left(r_{0}, r_{0}, r_{1}, r_{1}\right)+r_{0}^{2} I_{d / 2+2,3}\left(r_{0}, r_{0}, r_{0}, r_{1}\right)\right] E_{r_{0}} v^{\mu} E_{r_{1}},
$$

$\frac{d+2}{2}\left(v^{\mu} \otimes u \otimes \partial_{\mu} u\right) \rightsquigarrow \frac{d+2}{2} r_{1} I_{d / 2+2,3}\left(r_{0}, r_{1}, r_{1}, r_{2}\right) E_{r_{0}} v^{\mu} E_{r_{1}}\left(\partial_{\mu} u\right) E_{r_{2}}$, $\frac{d+2}{2}\left(u \otimes \partial_{\mu} u \otimes v^{\mu}\right) \rightsquigarrow \frac{d+2}{2} r_{0} I_{d / 2+2,3}\left(r_{0}, r_{0}, r_{1}, r_{2}\right) E_{r_{0}}\left(\partial_{\mu} u\right) E_{r_{1}} v^{\mu} E_{r_{2}}$, $\frac{d+2}{2}\left(u \otimes v^{\mu} \otimes \partial_{\mu} u\right) \rightsquigarrow \frac{d+2}{2} r_{0} I_{d / 2+2,3}\left(r_{0}, r_{0}, r_{1}, r_{2}\right) E_{r_{0}} v^{\mu} E_{r_{1}}\left(\partial_{\mu} u\right) E_{r_{2}}$.

For $k=4$, one has:

$$
\begin{gathered}
3\left[-\frac{1}{2} g^{\mu \nu} g_{\rho \sigma} g_{\alpha \beta}\left(\partial_{\mu} g^{\rho \sigma}\right)\left(\partial_{\nu} g^{\alpha \beta}\right)-g^{\mu \nu} g_{\rho \sigma} g_{\alpha \beta}\left(\partial_{\mu} g^{\rho \alpha}\right)\left(\partial_{\nu} g^{\sigma \beta}\right)-2 g_{\rho \sigma}\left(\partial_{\mu} g^{\mu \nu}\right)\left(\partial_{\nu} g^{\rho \sigma}\right)\right. \\
\left.-2 g_{\rho \sigma}\left(\partial_{\mu} g^{\mu \rho}\right)\left(\partial_{\nu} g^{\nu \sigma}\right)-2 g_{\rho \sigma}\left(\partial_{\mu} g^{\nu \rho}\right)\left(\partial_{\nu} g^{\mu \sigma}\right)\right] u \otimes u \otimes u \otimes u \\
\rightsquigarrow 3\left[-\frac{1}{2} g^{\mu \nu} g_{\rho \sigma} g_{\alpha \beta}\left(\partial_{\mu} g^{\rho \sigma}\right)\left(\partial_{\nu} g^{\alpha \beta}\right)-g^{\mu \nu} g_{\rho \sigma} g_{\alpha \beta}\left(\partial_{\mu} g^{\rho \alpha}\right)\left(\partial_{\nu} g^{\sigma \beta}\right)-2 g_{\rho \sigma}\left(\partial_{\mu} g^{\mu \nu}\right)\left(\partial_{\nu} g^{\rho \sigma}\right)\right. \\
\left.\quad-2 g_{\rho \sigma}\left(\partial_{\mu} g^{\mu \rho}\right)\left(\partial_{\nu} g^{\nu \sigma}\right)-2 g_{\rho \sigma}\left(\partial_{\mu} g^{\nu \rho}\right)\left(\partial_{\nu} g^{\mu \sigma}\right)\right] r_{0}^{4} I_{d / 2+3,4}\left(r_{0}, r_{0}, r_{0}, r_{0}, r_{0}\right) E_{r_{0}}, \\
-(d+4)\left[\frac{1}{2} g^{\mu \nu} g_{\rho \sigma}\left(\partial_{\nu} g^{\rho \sigma}\right)+\left(\partial_{\nu} g^{\mu \nu}\right)\right]\left[3 u \otimes u \otimes u \otimes \partial_{\mu} u\right. \\
\left.\quad+2 u \otimes u \otimes \partial_{\mu} u \otimes u+u \otimes \partial_{\mu} u \otimes u \otimes u\right] \\
\rightsquigarrow \quad-(d+4)\left[\frac{1}{2} g^{\mu \nu} g_{\rho \sigma}\left(\partial_{\nu} g^{\rho \sigma}\right)+\left(\partial_{\nu} g^{\mu \nu}\right)\right]\left[3 r_{0}^{3} I_{d / 2+3,4}\left(r_{0}, r_{0}, r_{0}, r_{0}, r_{1}\right)\right. \\
\left.\quad+2 r_{0}^{2} r_{1} I_{d / 2+3,4}\left(r_{0}, r_{0}, r_{0}, r_{1}, r_{1}\right)+r_{0} r_{1}^{2} I_{d / 2+3,4}\left(r_{0}, r_{0}, r_{1}, r_{1}, r_{1}\right)\right] E_{r_{0}}\left(\partial_{\mu} u\right) E_{r_{1}}, \\
-\frac{1}{2}(d+4)(d+2) g^{\mu \nu}\left(2 u \otimes u \otimes \partial_{\mu} u \otimes \partial_{\nu} u+u \otimes \partial_{\mu} u \otimes u \otimes \partial_{\nu} u\right) \\
\rightsquigarrow-\frac{1}{2}(d+4)(d+2) g^{\mu \nu}\left[2 r_{0}^{2} I_{d / 2+3,4}\left(r_{0}, r_{0}, r_{0}, r_{1}, r_{2}\right)+r_{0} r_{1} I_{d / 2+3,4}\left(r_{0}, r_{0}, r_{1}, r_{1}, r_{2}\right)\right] \\
E_{r_{0}}\left(\partial_{\mu} u\right) E_{r_{1}}\left(\partial_{\nu} u\right) E_{r_{2}} .
\end{gathered}
$$


The coefficient $\alpha$ and the spectral functions $F$ are evaluated by collecting these terms:

$$
\begin{aligned}
& \alpha:=\frac{1}{3}\left(\partial_{\mu} \partial_{\nu} g^{\mu \nu}\right)-\frac{1}{12} g^{\mu \nu} g_{\rho \sigma}\left(\partial_{\mu} \partial_{\nu} g^{\rho \sigma}\right)+\frac{1}{48} g^{\mu \nu} g_{\rho \sigma} g_{\alpha \beta}\left(\partial_{\mu} g^{\rho \sigma}\right)\left(\partial_{\nu} g^{\alpha \beta}\right) \\
& +\frac{1}{24} g^{\mu \nu} g_{\rho \sigma} g_{\alpha \beta}\left(\partial_{\mu} g^{\rho \alpha}\right)\left(\partial_{\nu} g^{\sigma \beta}\right)-\frac{1}{12} g_{\rho \sigma}\left(\partial_{\mu} g^{\mu \nu}\right)\left(\partial_{\nu} g^{\rho \sigma}\right) \\
& +\frac{1}{12} g_{\rho \sigma}\left(\partial_{\mu} g^{\nu \rho}\right)\left(\partial_{\nu} g^{\mu \sigma}\right)-\frac{1}{4} g_{\rho \sigma}\left(\partial_{\mu} g^{\mu \rho}\right)\left(\partial_{\nu} g^{\nu \sigma}\right), \\
& F_{w}\left(r_{0}, r_{1}\right):=I_{d / 2,1}\left(r_{0}, r_{1}\right) \text {, } \\
& F_{\partial v}\left(r_{0}, r_{1}\right):=-r_{0} I_{d / 2+1,2}\left(r_{0}, r_{0}, r_{1}\right) \text {, } \\
& F_{\partial \partial u}\left(r_{0}, r_{1}\right):=-\frac{d}{2} r_{0} I_{d / 2+1,2}\left(r_{0}, r_{0}, r_{1}\right)+(d+2) r_{0}^{2} I_{d / 2+2,3}\left(r_{0}, r_{0}, r_{0}, r_{1}\right), \\
& F_{\partial u}^{\mu}\left(r_{0}, r_{1}\right):=-\alpha^{\mu} r_{0} I_{d / 2+1,2}\left(r_{0}, r_{0}, r_{1}\right)+(d+6)\left[\frac{1}{2} \alpha^{\mu}+\beta^{\mu}\right] r_{0}^{2} I_{d / 2+2,3}\left(r_{0}, r_{0}, r_{0}, r_{1}\right) \\
& +\left[\frac{d+4}{2} \alpha^{\mu}+2 \beta^{\mu}\right] r_{0} r_{1} I_{d / 2+2,3}\left(r_{0}, r_{0}, r_{1}, r_{1}\right) \\
& -(d+4)\left[\frac{1}{2} \alpha^{\mu}+\beta^{\mu}\right]\left[3 r_{0}^{3} I_{d / 2+3,4}\left(r_{0}, r_{0}, r_{0}, r_{0}, r_{1}\right)\right. \\
& \left.+2 r_{0}^{2} r_{1} I_{d / 2+3,4}\left(r_{0}, r_{0}, r_{0}, r_{1}, r_{1}\right)+r_{0} r_{1}^{2} I_{d / 2+3,4}\left(r_{0}, r_{0}, r_{1}, r_{1}, r_{1}\right)\right] \text {, } \\
& F_{v, \mu}\left(r_{0}, r_{1}\right):=-\frac{1}{2} \alpha_{\mu} r_{1} I_{d / 2+1,2}\left(r_{0}, r_{1}, r_{1}\right)+\left[\frac{1}{2} \alpha_{\mu}+\beta_{\mu}\right]\left[r_{1}^{2} I_{d / 2+2,3}\left(r_{0}, r_{1}, r_{1}, r_{1}\right)\right. \\
& \left.+r_{0} r_{1} I_{d / 2+2,3}\left(r_{0}, r_{0}, r_{1}, r_{1}\right)+r_{0}^{2} I_{d / 2+2,3}\left(r_{0}, r_{0}, r_{0}, r_{1}\right)\right] \text {, } \\
& F_{v, v}\left(r_{0}, r_{1}, r_{2}\right):=-\frac{1}{2} I_{d / 2+1,2}\left(r_{0}, r_{1}, r_{2}\right) \text {, } \\
& F_{\partial u, v}\left(r_{0}, r_{1}, r_{2}\right):=\frac{d+2}{2} r_{0} I_{d / 2+2,3}\left(r_{0}, r_{0}, r_{1}, r_{2}\right), \\
& F_{v, \partial u}\left(r_{0}, r_{1}, r_{2}\right):=-\frac{d}{2} I_{d / 2+1,2}\left(r_{0}, r_{1}, r_{2}\right)+\frac{d+2}{2} r_{1} I_{d / 2+2,3}\left(r_{0}, r_{1}, r_{1}, r_{2}\right) \\
& +\frac{d+2}{2} r_{0} I_{d / 2+2,3}\left(r_{0}, r_{0}, r_{1}, r_{2}\right) \text {, } \\
& F_{\partial u, \partial u}\left(r_{0}, r_{1}, r_{2}\right):=\frac{(d+2)^{2}}{2} r_{0} I_{d / 2+2,3}\left(r_{0}, r_{0}, r_{1}, r_{2}\right) \\
& -\frac{(d+4)(d+2)}{2}\left[2 r_{0}^{2} I_{d / 2+3,4}\left(r_{0}, r_{0}, r_{0}, r_{1}, r_{2}\right)+r_{0} r_{1} I_{d / 2+3,4}\left(r_{0}, r_{0}, r_{1}, r_{1}, r_{2}\right)\right] .
\end{aligned}
$$

As in [1, Section 4.3], the strategy to compute (2.20) could be to make the change of variables $\left(u, v^{\mu}, w\right) \mapsto\left(u, p^{\mu}, q\right)$. Here we use another strategy which simplifies the computation since it is based on (2.8), (2.9), and (2.10).

Indeed, as already noticed, one can apply verbatim the computation of the arguments and their contractions with $\partial_{\mu}$ replaced by $\nabla_{\mu}$, and at the same time, using $p^{\mu}+g^{\mu \nu}\left(\nabla_{\nu} u\right)-\left[\frac{1}{2} \alpha^{\mu}-\beta^{\mu}\right] u$ in place of $v^{\mu}$, and $q$ in place of $w$. So, (2.19) can be replaced by

$$
\begin{aligned}
& \alpha r_{0}^{-d / 2+1} E_{r_{0}}+F_{\partial u}^{\mu}\left(r_{0}, r_{1}\right) E_{r_{0}}\left(\nabla_{\mu} u\right) E_{r_{1}}+g^{\mu \nu} F_{\partial \partial u}\left(r_{0}, r_{1}\right) E_{r_{0}}\left(\nabla_{\mu} \nabla_{\nu} u\right) E_{r_{1}} \\
& +g^{\mu \nu} F_{\partial u, \partial u}\left(r_{0}, r_{1}, r_{2}\right) E_{r_{0}}\left(\nabla_{\mu} u\right) E_{r_{1}}\left(\nabla_{\nu} u\right) E_{r_{2}}+F_{w}\left(r_{0}, r_{1}\right) E_{r_{0}} w E_{r_{1}}+F_{v, \mu}\left(r_{0}, r_{1}\right) E_{r_{0}} v^{\mu} E_{r_{1}} \\
& +F_{v, \partial u}\left(r_{0}, r_{1}, r_{2}\right) E_{r_{0}} v^{\mu} E_{r_{1}}\left(\nabla_{\mu} u\right) E_{r_{2}}+F_{\partial u, v}\left(r_{0}, r_{1}, r_{2}\right) a E_{r_{0}}\left(\nabla_{\mu} u\right) E_{r_{1}} v^{\mu} E_{r_{2}} \\
& +g_{\mu \nu} F_{v, v}\left(r_{0}, r_{1}, r_{2}\right) E_{r_{0}} v^{\mu} E_{r_{1}} v^{\nu} E_{r_{2}}+F_{\partial v}\left(r_{0}, r_{1}\right) E_{r_{0}}\left(\nabla_{\mu} v^{\mu}\right) E_{r_{1}} .
\end{aligned}
$$

The next step is to replace $\nabla_{\mu}$ by $\widehat{\nabla}_{\mu}$. We use (2.2), (2.3) and

$$
\begin{aligned}
\nabla_{\mu} v^{\mu} & =\nabla_{\mu} p^{\mu}+\left(\partial_{\mu} g^{\mu \nu}\right)\left(\nabla_{\nu} u\right)+g^{\mu \nu}\left(\nabla_{\mu} \nabla_{\nu} u\right)-\left[\frac{1}{2} \partial_{\mu} \alpha^{\mu}-\partial_{\mu} \beta^{\mu}\right] u-\left[\frac{1}{2} \alpha^{\mu}-\beta^{\mu}\right]\left(\nabla_{\mu} u\right) \\
& =\nabla_{\mu} p^{\mu}+g^{\mu \nu}\left(\widehat{\nabla}_{\mu} \widehat{\nabla}_{\nu} u\right)+\beta^{\mu}\left(\widehat{\nabla}_{\mu} u\right)-\left[\frac{1}{2} \partial_{\mu} \alpha^{\mu}-\partial_{\mu} \beta^{\mu}\right] u \\
& =\widehat{\nabla}_{\mu} p^{\mu}+\frac{1}{2} \alpha_{\mu} p^{\mu}+g^{\mu \nu}\left(\widehat{\nabla}_{\mu} \widehat{\nabla}_{\nu} u\right)+\beta^{\mu}\left(\widehat{\nabla}_{\mu} u\right)-\left[\frac{1}{2} \partial_{\mu} \alpha^{\mu}-\partial_{\mu} \beta^{\mu}\right] u .
\end{aligned}
$$

This leads to a new expression containing the terms in (2.20), with the functions given in the list after Theorem 2.4, to which we have to add the two terms $G_{\widehat{\nabla} u}^{\mu}\left(r_{0}, r_{1}\right) E_{r_{0}}\left(\widehat{\nabla}_{\mu} u\right) E_{r_{1}}+G_{p, \mu}\left(r_{0}, r_{1}\right) E_{r_{0}} p^{\mu} E_{r_{1}}$, 
with

$$
\begin{aligned}
G_{\widehat{\nabla} u}^{\mu}\left(r_{0}, r_{1}\right)= & F_{\partial u}^{\mu}\left(r_{0}, r_{1}\right)+g^{\mu \nu} F_{v, \nu}\left(r_{0}, r_{1}\right)+\beta^{\mu} F_{\partial v}\left(r_{0}, r_{1}\right) \\
& +\left[\frac{1}{2} \alpha^{\mu}-\beta^{\mu}\right]\left[F_{\partial \partial u}\left(r_{0}, r_{1}\right)-r_{0} F_{v, \partial u}\left(r_{0}, r_{0}, r_{1}\right)-r_{1} F_{\partial u, v}\left(r_{0}, r_{1}, r_{1}\right)\right. \\
& \left.-r_{0} F_{v, v}\left(r_{0}, r_{0}, r_{1}\right)-r_{1} F_{v, v}\left(r_{0}, r_{1}, r_{1}\right)\right], \\
G_{p, \mu}\left(r_{0}, r_{1}\right)= & F_{v, \mu}\left(r_{0}, r_{1}\right)+\frac{1}{2} \alpha_{\mu} F_{\partial v}\left(r_{0}, r_{1}\right) \\
& \quad-\left[\frac{1}{2} \alpha_{\mu}-\beta_{\mu}\right]\left[r_{0} F_{v, v}\left(r_{0}, r_{0}, r_{1}\right)+r_{1} F_{v, v}\left(r_{0}, r_{1}, r_{1}\right)\right] .
\end{aligned}
$$

A direct computation performed using the expressions of the spectral functions $F$ in terms of $I_{d / 2,1}$ shows that

$$
G_{\widehat{\nabla} u}^{\mu}\left(r_{0}, r_{1}\right)=G_{p, \mu}\left(r_{0}, r_{1}\right)=0 .
$$

The coefficient in front of $r_{0}^{-d / 2+1} E_{r_{0}}$ is

$$
\frac{1}{6} R=\alpha-\frac{1}{4} \alpha^{\mu} \beta_{\mu}+\frac{1}{2} \beta^{\mu} \beta_{\mu}+\frac{1}{4} \partial_{\mu} \alpha^{\mu}-\frac{1}{2} \partial_{\mu} \beta^{\mu}-\frac{1}{16} \alpha_{\mu} \alpha^{\mu}+\frac{1}{4} \alpha_{\mu} \beta^{\mu}-\frac{1}{4} \beta_{\mu} \beta^{\mu}
$$

where $R$ is the scalar curvature of the metric $g$.

The spectral functions $G$ can be written in terms of $\log$ functions for $d=2$ (see [1, Cor. 3.3]), as Laurent polynomials for $d \geq 4$ even (see [1, Prop. 3.5]), and in terms of square roots of $r_{i}$ for $d$ odd (see [1, Prop. 3.4]). This completes the proof of Corollaries 2.7, 2.8, and 2.10.

\section{Applications to the noncommutative torus}

In this section, we first apply Theorem 2.4 to the noncommutative 2-torus at rational values of the deformation parameter $\theta$, for which it is known that we get a geometrical description in terms of sections of a fiber bundle. Some computations of $a_{2}(a, P)$ for specific operators $P$ have been performed at irrational values of $\theta$ to determine the so-called scalar curvature (our $\mathcal{R}_{2}$ ) $[6,7,8,9$, $10,11,13,14,15,16,17,18]$.

We now show that we can apply our general result at rational values of $\theta$ and get the same expressions for the scalar curvature $\mathcal{R}_{2}$ which appears to be written in terms of $\theta$-independent spectral functions. In particular, its expression is the same for rational and irrational $\theta$.

Let $\Theta \in M_{d}(\mathbb{R})$ be a skew-symmetric real matrix. The noncommutative $d$-dimensional torus $C\left(\mathbb{T}_{\Theta}^{d}\right)$ is defined as the universal unital $C^{*}$-algebra generated by unitaries $U_{k}, k=1, \ldots, d$, satisfying the relations

$$
U_{k} U_{\ell}=e^{2 i \pi \Theta_{k, \ell}} U_{\ell} U_{k} .
$$

This $C^{*}$-algebra contains, as a dense sub-algebra, the space of smooth elements for the natural action of the $d$-dimensional torus $\mathbb{T}^{d}$ on $C\left(\mathbb{T}_{\Theta}^{d}\right)$. This sub-algebra is described as elements in $C\left(\mathbb{T}_{\Theta}^{d}\right)$ with an expansion

$$
a=\sum_{\left(k_{i}\right) \in \mathbb{Z}^{d}} a_{k_{1}, \ldots, k_{d}} U_{1}^{k_{1}} \cdots U_{d}^{k_{d}}
$$

where the sequence $\left(a_{k_{1}, \ldots, k_{d}}\right)$ belongs to the Schwartz space $\mathcal{S}\left(\mathbb{Z}^{d}\right)$. We denote by $C^{\infty}\left(\mathbb{T}_{\Theta}^{d}\right)$ this algebra. The $C^{*}$-algebra $C\left(\mathbb{T}_{\Theta}^{d}\right)$ has a unique normalized faithful positive trace $\mathfrak{t}$ whose restriction on smooth elements is given by

$$
\mathfrak{t}\left(\sum_{\left(k_{i}\right) \in \mathbb{Z}^{d}} a_{k_{1}, \ldots, k_{d}} U_{1}^{k_{1}} \cdots U_{d}^{k_{d}}\right):=a_{0, \ldots, 0}
$$


This trace satisfies $\mathfrak{t}(\mathbb{1})=1$ where $\mathbb{1}$ in the unit element of $C\left(\mathbb{T}_{\Theta}^{d}\right)$. The smooth algebra $C^{\infty}\left(\mathbb{T}_{\Theta}^{d}\right)$ has $d$ canonical derivations $\delta_{\mu}, \mu=1, \ldots, d$, defined on the generators by

$$
\delta_{\mu}\left(U_{k}\right):=\delta_{\mu, k} i U_{k} .
$$

For any $a \in C\left(\mathbb{T}_{\Theta}^{d}\right)$, one has $\delta_{\mu}\left(a^{*}\right)=\left(\delta_{\mu} a\right)^{*}$ (real derivations).

Denote by $\mathcal{H}$ the Hilbert space of the GNS representation of $C\left(\mathbb{T}_{\Theta}^{d}\right)$ defined by $\mathfrak{t}$. Each derivation $\delta_{\mu}$ defines a unbounded operator on $\mathcal{H}$, denoted also by $\delta_{\mu}$, which satisfies $\delta_{\mu}^{\dagger}=-\delta_{\mu}$ (here ${ }^{\dagger}$ denotes the adjoint of the operator).

\subsection{The geometry of the rational noncommutative tori}

In the following, we consider the special case of even dimensional noncommutative tori, $d=2 \mathrm{~m}$, with

$$
\Theta=\left(\begin{array}{ccc}
\theta_{1} \chi & \cdots & 0 \\
0 & \ddots & 0 \\
0 & \cdots & \theta_{m} \chi
\end{array}\right), \text { where } \chi:=\left(\begin{array}{cc}
0 & 1 \\
-1 & 0
\end{array}\right),
$$

for a family of deformation parameters $\theta_{1}, \ldots, \theta_{m}$. Then

$$
C\left(\mathbb{T}_{\Theta}^{2 m}\right) \simeq C\left(\mathbb{T}_{\Theta_{1}}^{2}\right) \otimes \cdots \otimes C\left(\mathbb{T}_{\Theta_{m}}^{2}\right)
$$

When $d=2$ and $\theta=p / q$, where $p, q$ are relatively prime integers and $q>0$, it is known that $C\left(\mathbb{T}_{\Theta}^{2}\right) \simeq \Gamma\left(A_{\theta}\right)$ is isomorphic to the algebra of continuous sections of a fiber bundle $A_{\theta}$ in $M_{q}(\mathbb{C})$ algebras over a 2-torus $\mathbb{T}_{B}^{2}$, as recalled in Section A. Similarly, for $d=4$, with $\theta_{1}=p_{1} / q_{1}$ and $\theta_{2}=p_{2} / q_{2}, C\left(\mathbb{T}_{\Theta}^{4}\right)$ is the space of sections of a fiber bundle in $M_{q_{1} q_{2}}(\mathbb{C})$ algebras over a 4 -torus $\mathbb{T}_{B, 1}^{2} \times \mathbb{T}_{B, 2}^{2}$.

Moreover, in the identification $C^{\infty}\left(\mathbb{T}_{\Theta}^{2}\right) \simeq \Gamma^{\infty}\left(A_{\theta}\right)$, the two derivations $\delta_{\mu}$ are the two components of the unique flat connection $\nabla_{\mu}$ on $A_{\theta}$.

This geometrical description allows to use the results of Section 2 to compute $a_{2}(a, P)$ for a differential operator on $\mathcal{H}$ of the form $P=-g^{\mu \nu} u \delta_{\mu} \delta_{\nu}-\left[p^{\nu}+g^{\mu \nu}\left(\delta_{\mu} u\right)-\left(\frac{1}{2} \alpha^{\nu}-\beta^{\nu}\right) u\right] \delta_{\nu}-q$

\subsection{The noncommutative two torus}

In this section, we compute the coefficient $a_{2}(a, P)$ on the rational noncommutative two torus for a differential operator $P$ considered in $[6,8,9,7]$ for the irrational noncommutative two torus. Let us introduce the following notations.

Let $\tau=\tau_{1}+i \tau_{2} \in \mathbb{C}$ with non zero imaginary part. We consider the constant metric $g$ defined by

$$
g^{11}=1, \quad g^{12}=g^{21}=\Re(\tau)=\tau_{1}, \quad g^{22}=|\tau|^{2},
$$

with inverse matrix

$$
g_{11}=\frac{|\tau|^{2}}{\Im(\tau)^{2}}=\frac{|\tau|^{2}}{\tau_{2}^{2}}, \quad g_{12}=g_{21}=-\frac{\Re(\tau)}{\Im(\tau)^{2}}=-\frac{\tau_{1}}{\tau_{2}^{2}}, \quad g_{22}=\frac{1}{\Im(\tau)^{2}}=\frac{1}{\tau_{2}^{2}} .
$$

We will use the constant tensors

$$
\epsilon^{1}:=1, \quad \epsilon^{2}:=\tau, \quad \bar{\epsilon}^{1}=1, \quad \bar{\epsilon}^{2}=\bar{\tau}, \quad h^{\mu \nu}:=\bar{\epsilon}^{\mu} \epsilon^{\nu},
$$

which imply $h^{11}=1, h^{12}=\tau, h^{21}=\bar{\tau}, h^{22}=|\tau|^{2}$. Then the symmetric part of $h^{\mu \nu}$ is the metric, $g^{\mu \nu}=\frac{1}{2}\left(h^{\mu \nu}+h^{\nu \mu}\right)$, and $g_{\mu \nu} \bar{\epsilon}^{\mu} \bar{\epsilon}^{\nu}=0$. 
On the (GNS) Hilbert space $\mathcal{H}$, consider the following operators $\delta, \delta^{\dagger}$ and the Laplacian:

$$
\begin{gathered}
\delta:=\bar{\epsilon}^{\mu} \delta_{\mu}=\delta_{1}+\bar{\tau} \delta_{2}, \quad \delta^{\dagger}=-\epsilon^{\mu} \delta_{\mu}=-\delta_{1}-\tau \delta_{2} \\
\Delta:=\delta^{\dagger} \delta=-\epsilon^{\mu} \bar{\epsilon}^{\nu} \delta_{\mu} \delta_{\nu}=-h^{\mu \nu} \delta_{\mu} \delta_{\nu}=-g^{\mu \nu} \delta_{\mu} \delta_{\nu} .
\end{gathered}
$$

For $k \in C^{\infty}\left(\mathbb{T}_{\Theta}^{d}\right), k>0$, the operator $P$ is defined as

$$
P:=\left(\begin{array}{cc}
P_{1} & 0 \\
0 & P_{2}
\end{array}\right)
$$

with

$$
\begin{aligned}
P_{1} & :=k \Delta k=-g^{\mu \nu} k \delta_{\mu} \delta_{\nu} k=-g^{\mu \nu} k^{2} \delta_{\mu} \delta_{\nu}-2 g^{\mu \nu} k\left(\delta_{\nu} k\right) \delta_{\mu}-g^{\mu \nu} k\left(\delta_{\mu} \delta_{\nu} k\right) \\
& =:-u_{1} g^{\mu \nu} \delta_{\mu} \delta_{\nu}-v_{1}^{\mu} \delta_{\mu}-w_{1}, \\
P_{2} & :=\delta^{\dagger} k^{2} \delta=-\epsilon^{\nu} \bar{\epsilon}^{\mu} \delta_{\nu} k^{2} \delta_{\mu}=-\bar{\epsilon}^{\mu} \epsilon^{\nu}\left(\delta_{\nu} k^{2}\right) \delta_{\mu}-\bar{\epsilon}^{\mu} \epsilon^{\nu} k^{2} \delta_{\mu} \delta_{\nu}=-g^{\mu \nu} k^{2} \delta_{\mu} \delta_{\nu}-h^{\mu \nu}\left(\delta_{\nu} k^{2}\right) \delta_{\mu} \\
& =:-u_{2} g^{\mu \nu} \delta_{\mu} \delta_{\nu}-v_{2}^{\mu} \delta_{\mu}-w_{2},
\end{aligned}
$$

so that

$$
\begin{aligned}
& u_{1}=k^{2}, \quad v_{1}^{\mu}=2 g^{\mu \nu} k\left(\delta_{\nu} k\right), \\
& u_{2}=k^{2}, \quad v_{2}^{\mu}=h^{\mu \nu}\left(\delta_{\nu} k^{2}\right)=h^{\mu \nu}\left[k\left(\delta_{\nu} k\right)+\left(\delta_{\nu} k\right) k\right], \quad w_{2}=0 .
\end{aligned}
$$

For the forthcoming computations, since the metric $g$ is constant, we have $\alpha=R=\alpha^{\mu}=\beta^{\mu}=0$, $\widehat{\nabla}_{\mu}=\nabla_{\mu}=\delta_{\mu}$ (the last equality being a property of the geometrical presentation of $C^{\infty}\left(\mathbb{T}_{\Theta}^{d}\right)$, as recalled above), $F_{\partial u}^{\mu}=F_{v, \mu}=0$. Here $|g|^{1 / 2}=\operatorname{det}\left(g_{\mu \nu}\right)^{1 / 2}=\tau_{2}^{-1}$. We can write $P_{1}$ and $P_{2}$ in the covariant form (2.1) with

$$
\begin{array}{lll}
u_{1}=k^{2}, & p_{1}^{\mu}=g^{\mu \nu}\left[k\left(\delta_{\nu} k\right)-\left(\delta_{\nu} k\right) k\right], & q_{1}=-k(\Delta k), \\
u_{2}=k^{2}, & p_{2}^{\mu}=\left(h^{\mu \nu}-g^{\mu \nu}\right)\left[k\left(\delta_{\nu} k\right)+\left(\delta_{\nu} k\right) k\right], & q_{2}=0 .
\end{array}
$$

Let

$$
f^{\mu \nu}:=\frac{1}{2}\left(h^{\mu \nu}-h^{\nu \mu}\right),
$$

so that $h^{\mu \nu}=g^{\mu \nu}+f^{\mu \nu}$ and $h^{\nu \mu}=g^{\mu \nu}-f^{\mu \nu}$, and define

$$
\begin{aligned}
Q_{g}(a, b, c) & :=\frac{\sqrt{a}(a \sqrt{b}+3 a \sqrt{c}-\sqrt{a} c-\sqrt{a b c}-2 b \sqrt{c})}{(a-b)(\sqrt{a}-\sqrt{b})(\sqrt{a}-\sqrt{c})^{3}}, \\
Q_{f}(a, b, c) & :=\frac{a(\sqrt{b}+\sqrt{c})}{(a-b)(\sqrt{a}-\sqrt{b})(a-c)},
\end{aligned}
$$

with the following (spectral) functions

$$
F_{\Delta k}\left(r_{0}, r_{1}\right)=\frac{r_{0}-r_{1}-\sqrt{r_{0} r_{1}}\left(\ln \left(r_{0}\right)-\ln \left(r_{1}\right)\right)}{\left(\sqrt{r_{0}}-\sqrt{r_{1}}\right)^{3}},
$$




$$
\begin{aligned}
F_{\partial k \partial k}^{\mu \nu}\left(r_{0}, r_{1}, r_{2}\right)= & \frac{g^{\mu \nu}\left(\sqrt{r_{0}}+\sqrt{r_{2}}\right)\left(\sqrt{r_{0}}-2 \sqrt{r_{1}}+\sqrt{r_{2}}\right)+f^{\mu \nu}\left(\sqrt{r_{0}}-\sqrt{r_{2}}\right)^{2}}{\left(\sqrt{r_{0}}-\sqrt{r_{1}}\right)\left(\sqrt{r_{0}}-\sqrt{r_{2}}\right)^{2}\left(\sqrt{r_{1}}-\sqrt{r_{2}}\right)} \\
& +\left[g^{\mu \nu} Q_{g}\left(r_{0}, r_{1}, r_{2}\right)-f^{\mu \nu} Q_{f}\left(r_{0}, r_{1}, r_{2}\right)\right] \ln \left(r_{0}\right) \\
& -\frac{\left(r_{1}+\sqrt{r_{0} r_{2}}\right)\left[g^{\mu \nu}\left(r_{1}+\sqrt{r_{0} r_{2}}\right)-f^{\mu \nu}\left(r_{1}-\sqrt{r_{0} r_{2}}\right)\right]}{\left(\sqrt{r_{0}}-\sqrt{r_{1}}\right)\left(r_{0}-r_{1}\right)\left(\sqrt{r_{1}}-\sqrt{r_{2}}\right)\left(r_{1}-r_{2}\right)} \ln \left(r_{1}\right) \\
& +\left[g^{\mu \nu} Q_{g}\left(r_{2}, r_{1}, r_{0}\right)-f^{\mu \nu} Q_{f}\left(r_{2}, r_{1}, r_{0}\right)\right] \ln \left(r_{2}\right) .
\end{aligned}
$$

With these notations:

Proposition 5.1 For the 2-dimensional noncommutative torus at rational values of the deformation parameter $\theta$, one has $a_{2}(a, P)=\varphi \circ L\left(a \mathcal{R}_{2}\right)$ for any $a \in C\left(\mathbb{T}_{\Theta}^{2}\right)$ with

$$
\mathcal{R}_{2}=: \frac{1}{4 \pi}\left[F_{\Delta k}\left(r_{0}, r_{1}\right) E_{r_{0}}(\Delta k) E_{r_{1}}+F_{\partial k \partial k}^{\mu \nu}\left(r_{0}, r_{1}, r_{2}\right) E_{r_{0}}\left(\delta_{\mu} k\right) E_{r_{1}}\left(\delta_{\nu} k\right) E_{r_{2}}\right] .
$$

where $L(a)$ is the left multiplication of a on $\mathcal{H}$ (GNS representation).

Since we are in dimension $d=2$, the appearance of the log function in this result is expected. It is shown in Appendix B that this result coincides with a previous one in [8, Theorem 5.2] for the irrational noncommutative two torus. As already mentioned, this computation, combined with results for irrational values of $\theta$, proves that $\mathcal{R}_{2}$ does not depend on the deformation parameter $\theta$, and in particular if it is irrational or not. The simplification of the $q$ 's in (A.2) (see also (A.3)) points out that the choice of the Hilbert space $\mathcal{H}$ as the GNS representation is a key fact because it permits to identify $\mathcal{R}_{2}$ in $(5.7)$ as an element in $C\left(\mathbb{T}_{\Theta}^{2}\right)$ acting by left multiplication on $\mathcal{H}$.

Nevertheless, the fact that $\mathcal{R}_{2}$ can be written in terms of $\theta$-independent spectral functions still needs an abstract proof. The spectrum of the differential operator $P$ depends on the differential operators $\delta_{\mu}$ and some multiplication operators by elements of the algebra (written here in terms of $k$ and its derivatives $\delta_{\mu} k, \delta_{\mu} \delta_{\nu} k$ ). On the one hand, the spectrum of the closed extension of the operator $\delta_{\mu}$ in the Hilbert space of the GNS representation consists only of eigenvalues $i k_{\mu}, k_{\mu} \in \mathbb{Z}$, associated to eigenvectors $U_{1}^{k_{1}} \cdots U_{d}^{k_{d}}$, so that it does not depend explicitly of $\theta$. On the other hand, the computations of $\mathcal{R}_{2}$, performed here or in $[6,8,9,7]$, are based on formal manipulations of the product in the algebra, in particular they do not use the defining relations (5.1). This explains why these methods bypass the $\theta$ dependency and give rise to some expressions in terms of $\theta$-independent spectral functions. Notice that for specific values of $\theta$, for instance $\theta=0$ (the commutative case), these expressions can be simplified. So, one has to look at (5.7) as a " $\theta$ universal" expression for $\mathcal{R}_{2}$.

Proof One has $a_{2}(a, P)=a_{2}\left(a, P_{1}\right)+a_{2}\left(a, P_{2}\right)$. Denote by $\mathcal{R}_{2}\left(\right.$ resp. $\left.\mathcal{R}_{2}^{(1)}, \mathcal{R}_{2}^{(2)}\right)$ the expressions associated to $P$ (resp. $P_{1}, P_{2}$ ). Then one has $\mathcal{R}_{2}=\mathcal{R}_{2}^{(1)}+\mathcal{R}_{2}^{(2)}$.

The operator $P_{1}$ is a conformal like transformed Laplacian, so the computation of $\mathcal{R}_{2}^{(1)}$ is a direct consequence of the results of Section 3.3. Here the metric is constant, so that $R=0$, and it remains

$$
\mathcal{R}_{2}^{(1)}=: \frac{1}{4 \pi}\left[F_{(1) \Delta k}\left(r_{0}, r_{1}\right) E_{r_{0}}(\Delta k) E_{r_{1}}+F_{(1) \partial k \partial k}^{\mu \nu}\left(r_{0}, r_{1}, r_{2}\right) E_{r_{0}}\left(\delta_{\mu} k\right) E_{r_{1}}\left(\delta_{\nu} k\right) E_{r_{2}}\right]
$$

where, using (3.2) and (3.3),

$$
F_{(1) \Delta k}\left(r_{0}, r_{1}\right)=-\sqrt{r_{0}} G_{q}\left(r_{0}, r_{1}\right)-\left(\sqrt{r_{0}}+\sqrt{r_{1}}\right) G_{\widehat{\nabla} \widehat{\nabla} u}\left(r_{0}, r_{1}\right)-\left(\sqrt{r_{0}}-\sqrt{r_{1}}\right) G_{\widehat{\nabla} p}\left(r_{0}, r_{1}\right),
$$




$$
\begin{aligned}
F_{(1) \partial k \partial k}^{\mu \nu}\left(r_{0}, r_{1}, r_{2}\right)= & 2 g^{\mu \nu} G_{\widehat{\nabla} \widehat{\nabla} u}\left(r_{0}, r_{2}\right) \\
& +g^{\mu \nu}\left(\sqrt{r_{0}}+\sqrt{r_{1}}\right)\left(\sqrt{r_{1}}+\sqrt{r_{2}}\right) G_{\widehat{\nabla} u, \widehat{\nabla} u}\left(r_{0}, r_{1}, r_{2}\right) \\
& +g^{\mu \nu}\left(\sqrt{r_{0}}-\sqrt{r_{1}}\right)\left(\sqrt{r_{1}}+\sqrt{r_{2}}\right) G_{p, \widehat{\nabla} u}\left(r_{0}, r_{1}, r_{2}\right) \\
& +g^{\mu \nu}\left(\sqrt{r_{0}}+\sqrt{r_{1}}\right)\left(\sqrt{r_{1}}-\sqrt{r_{2}}\right) G_{\widehat{\nabla} u, p}\left(r_{0}, r_{1}, r_{2}\right) \\
& +g^{\mu \nu}\left(\sqrt{r_{0}}-\sqrt{r_{1}}\right)\left(\sqrt{r_{1}}-\sqrt{r_{2}}\right) G_{p, p}\left(r_{0}, r_{1}, r_{2}\right) .
\end{aligned}
$$

For the operator $P_{2}$, one applies Theorem 2.4:

$$
\begin{aligned}
\mathcal{R}_{2}^{(2)}=\frac{1}{4 \pi}[ & \left(\sqrt{r_{0}}+\sqrt{r_{1}}\right) G_{\widehat{\nabla} \widehat{\nabla} u}\left(r_{0}, r_{1}\right) g^{\mu \nu} E_{r_{0}}\left(\delta_{\mu} \delta_{\nu} k\right) E_{r_{1}}+2 G_{\widehat{\nabla} \widehat{\nabla} u}\left(r_{0}, r_{2}\right) g^{\mu \nu} E_{r_{0}}\left(\delta_{\mu} k\right) E_{r_{1}}\left(\delta_{\nu} k\right) E_{r_{2}} \\
& +\left(\sqrt{r_{0}}+\sqrt{r_{1}}\right)\left(\sqrt{r_{1}}+\sqrt{r_{2}}\right) G_{\widehat{\nabla} u, \widehat{\nabla} u}\left(r_{0}, r_{1}, r_{2}\right) g^{\mu \nu} E_{r_{0}}\left(\delta_{\mu} k\right) E_{r_{1}}\left(\delta_{\nu} k\right) E_{r_{2}} \\
& +\left(\sqrt{r_{0}}+\sqrt{r_{1}}\right)\left(\sqrt{r_{1}}+\sqrt{r_{2}}\right) G_{p, \widehat{\nabla} u}\left(r_{0}, r_{1}, r_{2}\right)\left(h^{\nu \mu}-g^{\mu \nu}\right) E_{r_{0}}\left(\delta_{\mu} k\right) E_{r_{1}}\left(\delta_{\nu} k\right) E_{r_{2}} \\
& +\left(\sqrt{r_{0}}+\sqrt{r_{1}}\right)\left(\sqrt{r_{1}}+\sqrt{r_{2}}\right) G_{\widehat{\nabla} u, p}\left(r_{0}, r_{1}, r_{2}\right)\left(h^{\mu \nu}-g^{\mu \nu}\right) E_{r_{0}}\left(\delta_{\mu} k\right) E_{r_{1}}\left(\delta_{\nu} k\right) E_{r_{2}} \\
& \left.-\left(\sqrt{r_{0}}+\sqrt{r_{1}}\right)\left(\sqrt{r_{1}}+\sqrt{r_{2}}\right) G_{p, p}\left(r_{0}, r_{1}, r_{2}\right) g^{\mu \nu} E_{r_{0}}\left(\delta_{\mu} k\right) E_{r_{1}}\left(\delta_{\nu} k\right) E_{r_{2}}\right] \\
=: \frac{1}{4 \pi}[ & \left.F_{(2) \Delta k}\left(r_{0}, r_{1}\right) E_{r_{0}}(\Delta k) E_{r_{1}}+F_{(2) \partial k \partial k}^{\mu \nu}\left(r_{0}, r_{1}, r_{2}\right) E_{r_{0}}\left(\delta_{\mu} k\right) E_{r_{1}}\left(\delta_{\nu} k\right) E_{r_{2}}\right],
\end{aligned}
$$

with

$$
\begin{aligned}
F_{(2) \Delta k}\left(r_{0}, r_{1}\right)= & -\left(\sqrt{r_{0}}+\sqrt{r_{1}}\right) G_{\widehat{\nabla} \widehat{\nabla} u}\left(r_{0}, r_{1}\right), \\
F_{(2) \partial k \partial k}^{\mu \nu}\left(r_{0}, r_{1}, r_{2}\right)= & g^{\mu \nu} G_{\widehat{\nabla} \widehat{\nabla} u}\left(r_{0}, r_{2}\right) \\
& +g^{\mu \nu}\left(\sqrt{r_{0}}+\sqrt{r_{1}}\right)\left(\sqrt{r_{1}}+\sqrt{r_{2}}\right) G_{\widehat{\nabla} u, \widehat{\nabla} u}\left(r_{0}, r_{1}, r_{2}\right) \\
& +\left(h^{\nu \mu}-g^{\mu \nu}\right)\left(\sqrt{r_{0}}+\sqrt{r_{1}}\right)\left(\sqrt{r_{1}}+\sqrt{r_{2}}\right) G_{p, \widehat{\nabla} u}\left(r_{0}, r_{1}, r_{2}\right) \\
& +\left(h^{\mu \nu}-g^{\mu \nu}\right)\left(\sqrt{r_{0}}+\sqrt{r_{1}}\right)\left(\sqrt{r_{1}}+\sqrt{r_{2}}\right) G_{\widehat{\nabla} u, p}\left(r_{0}, r_{1}, r_{2}\right) \\
& -g^{\mu \nu}\left(\sqrt{r_{0}}+\sqrt{r_{1}}\right)\left(\sqrt{r_{1}}+\sqrt{r_{2}}\right) G_{p, p}\left(r_{0}, r_{1}, r_{2}\right) .
\end{aligned}
$$

Then $F_{\Delta k}:=F_{1, \Delta k}+F_{2, \Delta k}$ and $F_{\partial k \partial k}^{\mu \nu}:=F_{1, \partial k \partial k}^{\mu \nu}+F_{2, \partial k \partial k}^{\mu \nu}$ simplifies as in (5.5) and (5.6). The expression obtained for $\mathcal{R}_{2}$ shows that it belongs to $C\left(\mathbb{T}_{\Theta}^{2}\right)$ and acts by left multiplication on $\mathcal{H}$.

\subsection{The noncommutative four torus}

Our result applies to the computation of the conformally perturbed scalar curvature on the noncommutative four torus, computed in $[11,14]$. In order to do that, as in dimension 2 , we perform the computation at rational value of $\theta$ as described at the end of Appendix A.

The operator we consider is the one in [11], written as

$$
\Delta_{\varphi}:=k^{2} \bar{\partial}_{1} k^{-2} \partial_{1} k^{2}+k^{2} \partial_{1} k^{-2} \bar{\partial}_{1} k^{2}+k^{2} \bar{\partial}_{2} k^{-2} \partial_{2} k^{2}+k^{2} \partial_{2} k^{-2} \bar{\partial}_{2} k^{2}
$$

with (in our notations) $k^{2}:=e^{h}, \partial_{1}:=-\delta_{1}+i \delta_{3}, \bar{\partial}_{1}:=-\delta_{1}-i \delta_{3}, \partial_{2}:=-\delta_{2}+i \delta_{4}$, and $\bar{\partial}_{2}:=-\delta_{2}-i \delta_{4}$. Indeed, in $[11,14]$, the derivations are $\widehat{\delta}_{\mu}=-i \delta_{\mu}$. This leads to

$$
\begin{aligned}
\Delta_{\varphi} & =-2\left[k^{2} g^{\mu \nu} \delta_{\mu} \delta_{\nu}+g^{\mu \nu}\left(\delta_{\nu} k^{2}\right) \delta_{\mu}+g^{\mu \nu}\left(\delta_{\mu} \delta_{\nu} k^{2}\right)-g^{\mu \nu}\left(\delta_{\mu} k^{2}\right) k^{-2}\left(\delta_{\nu} k^{2}\right)\right] \\
& =: 2 P .
\end{aligned}
$$

The metric $g^{\mu \nu}$ is the diagonal one in [11], but in the following computation, we only require $g^{\mu \nu}$ to be constant. 
Let us mention that in $[11,14]$, the computation of the scalar curvature is done using $P$ defined above (and not $\Delta_{\varphi}$ ), since the symbol in [11, Lemma 3.6] is the one of $P$. So we will use $P$ in the following. We get $P=:-u g^{\mu \nu} \delta_{\mu} \delta_{\nu}-v^{\mu} \delta_{\mu}-w$ with

$$
u=k^{2}, \quad v^{\mu}=g^{\mu \nu}\left(\delta_{\nu} k^{2}\right), \quad w=g^{\mu \nu}\left(\delta_{\mu} \delta_{\nu} k^{2}\right)-g^{\mu \nu}\left(\delta_{\mu} k^{2}\right) k^{-2}\left(\delta_{\nu} k^{2}\right) .
$$

Since $g$ is constant, we have as before $\alpha=R=\alpha^{\mu}=\beta^{\mu}=0$ and $\widehat{\nabla}_{\mu}=\nabla_{\mu}=\delta_{\mu}$ and this implies $p^{\mu}=0$ and $q=g^{\mu \nu}\left(\delta_{\mu} \delta_{\nu} k^{2}\right)-g^{\mu \nu}\left(\delta_{\mu} k^{2}\right) k^{-2}\left(\delta_{\nu} k^{2}\right)$ in the covariant form (2.1). We then use the result of Corollary 2.9 to get the conformally perturbed scalar curvature:

$$
\mathcal{R}_{2}=\frac{1}{2^{5} \pi^{2}}\left[g^{\mu \nu} k^{-2}\left(\delta_{\mu} \delta_{\nu} k^{2}\right) k^{-2}-\frac{3}{2} g^{\mu \nu} k^{-2}\left(\delta_{\mu} k^{2}\right) k^{-2}\left(\delta_{\nu} k^{2}\right) k^{-2}\right] .
$$

In Appendix B it is shown that we recover the result previouly obtained in $[11,14]$ for the irrational noncommutative four torus.

\section{Conclusion}

In this paper, we have computed in all dimensions the local section $\mathcal{R}_{2}$ of $\operatorname{End}(V)$ defined by $a_{2}(a, P)=\int_{M} \operatorname{tr}\left[a(x) \mathcal{R}_{2}(x)\right] \operatorname{dvol}_{g}(x)$ for any section $a$ of $\operatorname{End}(V)$ for any nonminimal Laplace type operator $P=-\left[g^{\mu \nu} u(x) \partial_{\mu} \partial_{\nu}+v^{\nu}(x) \partial_{\nu}+w(x)\right]$ (Theorems 2.3 and 2.4). Expressions have been given for $\mathcal{R}_{2}$ in small dimensions, $d=2,3,4$ (Corollaries 2.7, 2.9, and 2.10) and for any even dimension $d \geq 2$ (Corollary 2.8), where, as expected from the results in [1], polynomials expressions can be proposed.

Despite the difficulties, $a_{4}(a, P)$ has been exhibited for $d=2$ in [18] for the 2-dimensional noncommutative torus, leaving open the computation of $\mathcal{R}_{4}$. We hope that our method could be used to reach $\mathcal{R}_{4}$ in any dimension, using a computer algebra system in the more general framework of an arbitrary $P$, like (1.1).

Our method still applies to more general setting than the NCT at rational values of the deformation parameter, namely to $n$-homogeneous $C^{*}$-algebras, which can be characterized in terms of sections of fiber bundles with fiber space $M_{n}(\mathbb{C})[24,25]$.

\section{Acknowledgements}

The authors are indebted to Valentin Zagrebnov for helpful discussions concerning some aspects of this paper.

\section{A. Geometrical identification of the noncommutative torus at rational values}

For $d=2$ and $\theta:=p / q$ rational, with $p, q$ relatively prime integers with $q>0$, it is known (see [26, Prop. 12.2], [27, Sect. 3]) that the algebra $C\left(\mathbb{T}_{\Theta}^{2}\right)$ of the NCT identifies with the algebra $\Gamma\left(A_{\theta}\right)$ of continuous sections of a fiber bundle $A_{\theta}$ in $M_{q}(\mathbb{C})$ algebras over a 2-torus $\mathbb{T}_{B}^{2}$. Let us describe this identification.

Denote by $\mathbb{T}_{P}^{2}$ the 2 -torus given by identification of opposite sides of the square $[0,2 \pi]^{2}$. An element in $\mathbb{T}_{P}^{2}$ is written as $\left(e^{i x}, e^{i y}\right)$ for $(x, y) \in[0,2 \pi]^{2}$. There is a natural action of the (abelian discrete) group $G:=\mathbb{Z}_{q}^{2}$ on $\mathbb{T}_{P}^{2}:(m, n) \cdot\left(e^{i x}, e^{i y}\right):=\left(e^{i(x+2 \pi p m / q)}, e^{i(y+2 \pi p n / q)}\right)$.

The quotient $\mathbb{T}_{B}^{2}:=\mathbb{T}_{P}^{2} / G$ is the 2-torus constructed by identification of opposite sides of the square $[0,2 \pi / q]^{2}$. Indeed, there are unique $m \in \mathbb{Z}_{q}$ and $n \in \mathbb{Z}_{q}$ such that $e^{i(x+2 \pi p m / q)}=e^{i(x+2 \pi / q)}$ and 
$e^{i(y+2 \pi p n / q)}=e^{i(y+2 \pi / q)}$, so that $(m, 0)$ (resp. $\left.(0, n)\right)$ identifies $\left(e^{i x}, e^{i y}\right)$ with $\left(e^{i(x+2 \pi / q)}, e^{i y}\right)$ (resp. $\left(e^{i x}, e^{i y}\right)$ with $\left.\left(e^{i x}, e^{i(y+2 \pi / q)}\right)\right)$ in $\mathbb{T}_{P}^{2} / G$. The quotient map $\mathbb{T}_{P}^{2} \rightarrow \mathbb{T}_{B}^{2}$ is a $G$-covering.

Let us now consider the $C^{*}$-algebra $C\left(\mathbb{T}_{P}^{2}, M_{q}(\mathbb{C})\right) \simeq C\left(\mathbb{T}_{P}^{2}\right) \otimes M_{q}(\mathbb{C})$ of matrix-valued continuous functions on $\mathbb{T}_{P}^{2}$, in which the space of smooth functions $C^{\infty}\left(\mathbb{T}_{P}^{2}, M_{q}(\mathbb{C})\right)$ is a dense subalgebra. In order to describe this algebra, let consider the two matrices

$$
U_{0}:=\left(\begin{array}{ccccc}
0 & 1 & 0 & \cdots & 0 \\
0 & 0 & 1 & \cdots & 0 \\
\vdots & & & \ddots & \vdots \\
0 & 0 & 0 & \cdots & 1 \\
1 & 0 & 0 & \cdots & 0
\end{array}\right), \quad V_{0}:=\left(\begin{array}{ccccc}
1 & 0 & 0 & \cdots & 0 \\
0 & \xi_{1} & 0 & \cdots & 0 \\
\vdots & & \ddots & & \vdots \\
0 & \cdots & 0 & \xi_{q-2} & 0 \\
0 & 0 & \cdots & 0 & \xi_{q-1}
\end{array}\right) \quad \text { with } \xi_{n}:=e^{i 2 \pi n \theta}
$$

which satisfy $U_{0} V_{0}=e^{i 2 \pi \theta} V_{0} U_{0}, U_{0}^{q}=V_{0}^{q}=\mathbb{1}_{q}$. For $(r, s) \in \mathbb{Z}_{q}^{2}$, the $U_{0}^{r} V_{0}^{s}$ 's define a basis of $M_{q}(\mathbb{C})$ such that $\operatorname{tr}\left[U_{0}^{r} V_{0}^{s}\right]=q \delta_{(r, s),(0,0)}$ (here tr is the trace on $M_{q}(\mathbb{C})$ ). Then $a \in C^{\infty}\left(\mathbb{T}_{P}^{2}, M_{q}(\mathbb{C})\right)$ can be decomposed as

$$
a\left(e^{i x}, e^{i y}\right)=\sum_{(r, s) \in \mathbb{Z}_{q}^{2}} a_{r, s}\left(e^{i x}, e^{i y}\right) U_{0}^{r} V_{0}^{s}=\sum_{(k, \ell) \in \mathbb{Z}^{2}} \sum_{(r, s) \in \mathbb{Z}_{q}^{2}} a_{k, \ell, r, s} u(x)^{k} v(x)^{\ell} U_{0}^{r} V_{0}^{s}
$$

where, with $u(x):=e^{i x}$ and $v(x):=e^{i y}$, the last decomposition is the Fourier series of the smooth functions $a_{r, s}$ on $\mathbb{T}_{P}^{2}$. In particular, $a_{k, \ell, r, s}$ are rapidly decreasing coefficients in terms of $(k, \ell) \in \mathbb{Z}^{2}$.

The group $G$ acts on $M_{q}(\mathbb{C})$ by

$$
(m, n) \cdot A:=U_{0}^{-n} V_{0}^{m} A V_{0}^{-m} U_{0}^{n} .
$$

Let us consider the subalgebra $C_{G}\left(\mathbb{T}_{P}^{2}, M_{q}(\mathbb{C})\right) \subset C\left(\mathbb{T}_{P}^{2}, M_{q}(\mathbb{C})\right)$ of $G$-equivariant functions, which by definition satisfy, for any $(m, n) \in G$,

$$
a\left(e^{i(x+2 \pi p m / q)}, e^{i(y+2 \pi p n / q)}\right)=U_{0}^{n} V_{0}^{-m} a\left(e^{i x}, e^{i y}\right) V_{0}^{m} U_{0}^{-n} .
$$

Using (A.1), the $G$-equivariant elements in $C^{\infty}\left(\mathbb{T}_{P}^{2}, M_{q}(\mathbb{C})\right)$ are such that their coefficients satisfy $a_{k, \ell, r, s} e^{i 2 \pi(m k+n \ell)}=a_{k, \ell, r, s} e^{i 2 \pi(m r+n s)}$ for any $(m, n) \in G,(k, \ell) \in \mathbb{Z}^{2}$ and $(r, s) \in \mathbb{Z}_{q}^{2}$, so that $a_{k, \ell, r, s} \neq 0$ only when $m k+n \ell \equiv m r+n s \bmod q$. With $(m, n)=(1,0)$ and $(0,1)$ this implies $k \equiv r \bmod q$ and $\ell \equiv s \bmod q$. In $(\mathrm{A} .1)$, for a couple $(k, \ell) \in \mathbb{Z}^{2}$, there is a unique $(r, s) \in \mathbb{Z}_{q}^{2}$ for which $a_{k, \ell, r, s} \neq 0$ ( $r$ and $s$ are the remainders of the Euclidean divisions of $k$ and $\ell$ by $q$ ). Then, the only non zero coefficients $a_{k, \ell, r, s}$ depends only on $(k, \ell) \in \mathbb{Z}^{2}$. We denote them by $a_{k, \ell}$, and a smooth $G$-equivariant function $a \in C_{G}^{\infty}\left(\mathbb{T}_{P}^{2}, M_{q}(\mathbb{C})\right)$ is then given by the expansion

$$
a=\sum_{(k, \ell) \in \mathbb{Z}^{2}} a_{k, \ell}\left(u U_{0}\right)^{k}\left(v V_{0}\right)^{\ell}=\sum_{(k, \ell) \in \mathbb{Z}^{2}} a_{k, \ell} U^{k} V^{\ell}
$$

with $U:=u U_{0}, V:=v V_{0}$ satisfying $U V=e^{i 2 \pi \theta} V U$. Then, the $C^{*}$-algebra $C\left(\mathbb{T}_{\Theta}^{2}\right)$ for $\theta=p / q$ identifies with $C_{G}\left(\mathbb{T}_{P}^{2}, M_{q}(\mathbb{C})\right)$, the $C^{*}$-completion of $C_{G}^{\infty}\left(\mathbb{T}_{P}^{2}, M_{q}(\mathbb{C})\right)$ in $C\left(\mathbb{T}_{P}^{2}, M_{q}(\mathbb{C})\right)$.

The space $C_{G}\left(\mathbb{T}_{P}^{2}, M_{q}(\mathbb{C})\right)$ identifies in a canonical way with the space $\Gamma\left(A_{\theta}\right)$ of continuous sections of the associated fiber bundle $A_{\theta}:=\mathbb{T}_{P}^{2} \times_{G} M_{q}(\mathbb{C})$ to the $G$-covering $\mathbb{T}_{P}^{2} \rightarrow \mathbb{T}_{B}^{2}$. By definition, $A_{\theta}$ is the quotient of $\mathbb{T}_{P}^{2} \times M_{q}(\mathbb{C})$ by the equivalence relation $\left((m, n) \cdot\left(e^{i x}, e^{i y}\right), A\right) \sim\left(\left(e^{i x}, e^{i y}\right),(m, n) \cdot A\right)$ for any $(m, n) \in G$. We denote by $\left[\left(e^{i x}, e^{i y}\right), A\right] \in A_{\theta}$ the class of $\left(\left(e^{i x}, e^{i y}\right), A\right)$. To $a \in C_{G}\left(\mathbb{T}_{P}^{2}, M_{q}(\mathbb{C})\right)$ corresponds the section $s \in \Gamma\left(A_{\theta}\right)$ defined by $s(x, y):=\left[\left(e^{i x}, e^{i y}\right), a\left(e^{i x}, e^{i y}\right)\right]$. 
In the GNS construction, $C_{G}^{\infty}\left(\mathbb{T}_{P}^{2}, M_{q}(\mathbb{C})\right)$ is dense in $\mathcal{H}$ and is contained in the domains of the $\delta_{\mu}$ 's. The fiber of the vector bundle $V$ on which the differential operator $P$ acts is then $\mathbb{C}^{N} \simeq M_{q}(\mathbb{C})$, i.e. $N=q^{2}$. In the present situation, all used elements in $\Gamma(\operatorname{End}(V))$ are in fact left multiplications by elements in $C_{G}\left(\mathbb{T}_{P}^{2}, M_{q}(\mathbb{C})\right) \simeq \Gamma\left(A_{\theta}\right)$. For instance, the element $a$ in (1.4) will be understood as the left multiplication by an element $a \in C_{G}\left(\mathbb{T}_{P}^{2}, M_{q}(\mathbb{C})\right)$.

For $A \in M_{q}(\mathbb{C})$, let $L(A)$ be the left multiplication by $A$ on $M_{q}(\mathbb{C})$. Then $L(A)$ has the same spectrum as $A$, each eigenvalues having $q$ times its original multiplicity. In particular, we have that

$$
\operatorname{tr}[L(A)]=q \operatorname{tr}[A]
$$

where in the LHS tr is the trace of operators on $M_{q}(\mathbb{C})$ and in the RHS tr is the trace on $M_{q}(\mathbb{C})$.

The computation of $\mathcal{R}_{2}$ uses local trivializations of sections of $A_{\theta}$. To $s \in \Gamma\left(A_{\theta}\right)$, we associate the local section $s_{\text {loc }}:(0,2 \pi / q)^{2} \rightarrow M_{q}(\mathbb{C})$ defined by $s_{\text {loc }}(x, y):=a\left(e^{i x}, e^{i y}\right)$. Notice that the open subset $(0,2 \pi / q)^{2} \subset \mathbb{T}_{B}^{2}$ is sufficient to describe the continuous section $s$ via its trivialization $s_{\text {loc }}$. The (local) section $\mathcal{R}_{2}$ relative to $\varphi$ in (1.4) is defined by

$$
\varphi\left(L\left(s_{\text {loc }}\right)\right)=\int_{\mathbb{T}_{B}^{2}} \operatorname{tr}\left[L\left(s_{\text {loc }}(x, y)\right)\right] \operatorname{dvol}_{g}(x, y)=|g|^{1 / 2} \int_{0}^{2 \pi / q} \mathrm{~d} x \int_{0}^{2 \pi / q} \mathrm{~d} y q \operatorname{tr}\left[s_{\text {loc }}(x, y)\right] .
$$

where we suppose here that $|g|^{1 / 2}$ is constant (this is the case for the situations considered in the paper). For $s$ associated to $a \in C_{G}^{\infty}\left(\mathbb{T}_{P}^{2}, M_{q}(\mathbb{C})\right)$, one has

$$
s_{\text {loc }}(x, y)=\sum_{(k, \ell) \in \mathbb{Z}^{2}} a_{k, \ell}\left(u(x) U_{0}\right)^{k}\left(v(x) V_{0}\right)^{\ell},
$$

hence

$$
\varphi\left(L\left(s_{\text {loc }}\right)\right)=|g|^{1 / 2} q \sum_{(k, \ell) \in \mathbb{Z}^{2}} a_{k, \ell} \int_{0}^{2 \pi / q} \mathrm{~d} x e^{i k x} \int_{0}^{2 \pi / q} \mathrm{~d} y e^{i \ell y} \operatorname{tr}\left[U_{0}^{k} V_{0}^{\ell}\right] .
$$

The trace $\operatorname{tr}\left[U_{0}^{k} V_{0}^{\ell}\right]$ is non-zero only when $k, \ell$ are multiple of $q$, and its value is then $q$, so that

$$
\varphi\left(L\left(s_{\text {loc }}\right)\right)=|g|^{1 / 2} q^{2} \sum_{(k, \ell) \in \mathbb{Z}^{2}} a_{k, \ell} \int_{0}^{2 \pi / q} \mathrm{~d} x e^{i q k x} \int_{0}^{2 \pi / q} \mathrm{~d} y e^{i q \ell y}=|g|^{1 / 2} q^{2}\left(\frac{2 \pi}{q}\right)^{2} a_{0,0}=(2 \pi)^{2}|g|^{1 / 2} \mathfrak{t}(a) .
$$

Finally we get (when $|g|^{1 / 2}$ is constant)

$$
\varphi \circ L=(2 \pi)^{2}|g|^{1 / 2} \mathfrak{t}
$$

when applied to any elements in $C\left(\mathbb{T}_{\Theta}^{2}\right)$.

Consider now a 4-dimensional noncommutative torus for $\Theta=\left(\begin{array}{cc}\theta_{1} \chi & 0 \\ 0 & \theta_{2} \chi\end{array}\right)$, as in (5.3), and $\theta_{i}=p_{i} / q_{i}$, $p_{i}, q_{i}$ relatively prime integers, and $q_{i}>0$.

Then $C\left(\mathbb{T}_{\Theta}^{4}\right)=C\left(\mathbb{T}_{\Theta_{1}}^{2}\right) \otimes C\left(\mathbb{T}_{\Theta_{2}}^{2}\right)=\Gamma\left(A_{\theta_{1}}\right) \otimes \Gamma\left(A_{\theta_{2}}\right)=\Gamma\left(A_{\theta_{1}} \otimes A_{\theta_{2}}\right)$ where $A_{\theta_{1}} \otimes A_{\theta_{2}}$ is the external tensor product of the two vector bundles $A_{\theta_{i}}$ over the base 2-torus $\mathbb{T}_{B, i}^{2}$ defined as above. Recall that, with $\operatorname{pr}_{i}: \mathbb{T}_{B, 1}^{2} \times \mathbb{T}_{B, 2}^{2} \rightarrow \mathbb{T}_{B, i}^{2}$ the natural projections, $A_{\theta_{1}} \otimes A_{\theta_{2}}:=\left(\operatorname{pr}_{1}^{*} A_{\theta_{1}}\right) \otimes\left(\operatorname{pr}_{2}^{*} A_{\theta_{2}}\right)$ where $\operatorname{pr}_{i}^{*} A_{\theta_{i}}$ is the pull-back of $A_{\theta_{i}}$ on $\mathbb{T}_{B, 1}^{2} \times \mathbb{T}_{B, 2}^{2}$. The fiber of $A_{\theta_{1}} \otimes A_{\theta_{2}}$ is then $M_{q_{1}}(\mathbb{C}) \otimes M_{q_{2}}(\mathbb{C}) \simeq M_{q_{1} q_{2}}(\mathbb{C})$ and the isomorphism $\Gamma\left(A_{\theta_{1}}\right) \otimes \Gamma\left(A_{\theta_{2}}\right) \stackrel{\simeq}{\rightarrow} \Gamma\left(A_{\theta_{1}} \otimes A_{\theta_{2}}\right)$ is induced by $\left(s_{1} \otimes s_{2}\right)\left(x_{1}, x_{2}\right):=s_{1}\left(x_{1}\right) \otimes s_{2}\left(x_{2}\right)$ 
for any $s_{i} \in \Gamma\left(A_{\theta_{i}}\right)$ and $x_{i} \in \mathbb{T}_{B, i}^{2}$. Using the same line of arguments as for the 2-dimensional case, and denoting by $g_{i}$ a constant metric on $\mathbb{T}_{B, i}^{2}$, one gets, for any $a_{i} \in C\left(\mathbb{T}_{\Theta_{i}}^{2}\right)$,

$$
\varphi \circ L\left(a_{1} \otimes a_{2}\right)=\left|g_{1}\right|^{1 / 2}\left|g_{2}\right|^{1 / 2}\left(q_{1} q_{2}\right)^{2}\left(\frac{2 \pi}{q_{1}}\right)^{2}\left(\frac{2 \pi}{q_{2}}\right)^{2} \mathfrak{t}\left(a_{1} \otimes a_{2}\right),
$$

so that

$$
\varphi \circ L=(2 \pi)^{4}\left|g_{1}\right|^{1 / 2}\left|g_{2}\right|^{1 / 2} \mathfrak{t} .
$$

This procedure can be extended straightforwardly to any even dimension.

\section{B. Comparison with previous results for noncommutative tori}

We would like to compare the result (5.7) with [8, Theorem 5.2]. Some transformations are in order, since some conventions are different and the results are presented using different operators. In [8, Theorem 5.2], it is presented relative to the normalized trace $\mathfrak{t}$ on $C\left(\mathbb{T}_{\Theta}^{2}\right)$, while our result is presented relative to $\varphi \circ L=(2 \pi)^{2}|g|^{1 / 2} \mathfrak{t}$ with here $|g|^{1 / 2}=\tau_{2}^{-1}$. If $\mathcal{R}_{F K}$ denotes the operator of [8, Theorem 5.2], then

$$
a_{2}(a, P)=\mathfrak{t}\left(a \mathcal{R}_{F K}\right)=\varphi\left(a \mathcal{R}_{2}\right)=(2 \pi)^{2} \tau_{2}^{-1} \mathfrak{t}\left(a \mathcal{R}_{2}\right), \quad \forall a \in C\left(\mathbb{T}_{\Theta}^{2}\right),
$$

so we need to show that $\mathcal{R}_{2}=\frac{\tau_{2}}{(2 \pi)^{2}} \mathcal{R}_{F K}$ (strictly speaking, $a \mathcal{R}_{2}$ should be replaced by $L\left(s \mathcal{R}_{2}\right)$ in $\varphi\left(a \mathcal{R}_{2}\right)$, see Section A).

Present results are given using functional calculus on the left and right multiplication operators $L_{u}$ and $R_{u}$ where $u=k^{2}$. The corresponding spectral decompositions give $L_{u}(a)=\sum_{r_{0}} r_{0} E_{r_{0}} a$ and $R_{u}(a)=\sum_{r_{1}} r_{1} a E_{r_{1}}$, where $E_{r_{i}}$ is the projection associated to $u$ for the spectral value $r_{i}$. In [8], another convention is used, namely via functional calculus on the modular operator $\boldsymbol{\Delta}(a):=k^{-2} a k^{2}$. If $E_{y}^{\Delta}$ denotes the projection of $\boldsymbol{\Delta}$ associated to the spectral value $y$, then

$$
\Delta(a)=L_{u^{-1}} \circ R_{u}(a)=\sum_{r_{0}, r_{1}} r_{0}^{-1} r_{1} E_{r_{0}} a E_{r_{1}}=\sum_{r_{0}, y} y E_{r_{0}} a E_{y r_{0}}=\sum_{y} y E_{y}^{\boldsymbol{\Delta}}(a)
$$

where $y:=r_{0}^{-1} r_{1}$ belongs to the spectrum of $\boldsymbol{\Delta}$.

Thus $E_{y}^{\boldsymbol{\Delta}}(a)=\sum_{r_{0}} E_{r_{0}} a E_{y r_{0}}$ and is easy to check that $E_{y_{0}}^{\boldsymbol{\Delta}} E_{y_{1}}^{\boldsymbol{\Delta}}=\delta_{y_{0}, y_{1}} E_{y_{0}}^{\boldsymbol{\Delta}}$.

The next three lemmas are technical results which permit to transform our relation (5.7) into a relation that will be compared to $[8$, Theorem 5.2].

For any $b_{0} \otimes \cdots \otimes b_{p} \in M_{N}(\mathbb{C})^{\otimes^{p+1}}$, we denote by $\boldsymbol{\Delta}_{i}$ the operator $\boldsymbol{\Delta}$ which acts on $b_{i}$, by $L_{k}^{i}$ the left multiplication by $k$ acting on $b_{i}$, and by $R_{u}^{i}$ the right multiplication by $u$ on $b_{i}$. Notice that all these operators commute.

The first lemma transforms the functional calculus in the $R_{u}^{i}$ 's into a functional calculus in the $\boldsymbol{\Delta}_{i}$ 's.

Lemma B.1 (Rearrangement Lemma) For any function $F\left(r_{0}, r_{1}, \ldots, r_{p}\right)$ of the eigenvalues $r_{i}$ of the $R_{u}^{i}$ 's and any $b_{0} \otimes \cdots \otimes b_{p}$ one has

$$
\sum_{r_{0}, r_{1}, \ldots, r_{p}} F\left(r_{0}, r_{1}, \ldots, r_{p}\right) b_{0} E_{r_{0}} b_{1} E_{r_{1}} \cdots b_{p} E_{r_{p}}=\sum_{r_{0}, y_{1}, \ldots, y_{p}} f\left(r_{0}, y_{1}, \ldots, y_{p}\right) b_{0} E_{r_{0}} E_{y_{1}}^{\Delta}\left(b_{1}\right) \cdots E_{y_{p}}^{\Delta}\left(b_{p}\right)
$$

where $f\left(r_{0}, y_{1}, \ldots, y_{p}\right):=F\left(r_{0}, r_{0} y_{1}, r_{0} y_{1} y_{2}, \ldots, r_{0} y_{1} \cdots y_{p}\right)$ is a spectral function of $R_{u}^{0}$ and the $\boldsymbol{\Delta}_{i}$ 's. 
Using functional calculus notation, this lemma implies

$$
\mathbf{m} \circ F\left(R_{u}^{0}, R_{u}^{1}, \ldots, R_{u}^{p}\right)=\mathbf{m} \circ f\left(R_{u}^{0}, \Delta_{1}, \ldots, \boldsymbol{\Delta}_{p}\right)
$$

as operators acting on elements $b_{0} \otimes \cdots \otimes b_{p}$. This result is very analog to the rearrangement lemma [28, Corollary 3.9] without the integral $\int_{0}^{\infty} d u$ in [28, eq. (3.9)].

Proof It is sufficient to show how the combinatorial aspect of the proof works for $p=2$. One has

$$
\begin{aligned}
\sum_{r_{0}, y_{1}, y_{2}} f\left(r_{0}, y_{1}, y_{2}\right) b_{0} E_{r_{0}} E_{y_{1}}^{\Delta}\left(b_{1}\right) E_{y_{2}}^{\Delta}\left(b_{2}\right) & =\sum_{r_{0}, y_{1}, y_{2}} F\left(r_{0}, r_{0} y_{1}, r_{0} y_{1} y_{2}\right) b_{0} E_{r_{0}} E_{y_{1}}^{\Delta}\left(b_{1}\right) E_{y_{2}}^{\Delta}\left(b_{2}\right) \\
& =\sum_{r_{0}, y_{1}, y_{2}} F\left(r_{0}, r_{0} y_{1}, r_{0} y_{1} y_{2}\right) b_{0} E_{r_{0}} E_{z_{1}} b_{1} E_{y_{1} z_{1}} E_{z_{2}} b_{2} E_{y_{2} z_{2}} \\
& =\sum_{\substack{r_{0}, y_{1}, y_{2} \\
z_{2}}} F\left(r_{0}, r_{0} y_{1}, r_{0} y_{1} y_{2}\right) b_{0} E_{r_{0}} b_{1} E_{y_{1} r_{0}} E_{z_{2}} b_{2} E_{y_{2} z_{2}} \\
& =\sum_{r_{0}, r_{1}, y_{2}} F\left(r_{0}, r_{1}, r_{1} y_{2}\right) b_{0} E_{r_{0}} b_{1} E_{r_{1}} E_{z_{2}} b_{2} E_{y_{2} z_{2}} \\
& =\sum_{r_{0}, r_{1}, y_{2}} F\left(r_{0}, r_{1}, r_{1} y_{2}\right) b_{0} E_{r_{0}} b_{1} E_{r_{1}} b_{2} E_{y_{2} r_{1}} \\
& =\sum_{r_{0}, r_{1}, r_{2}} F\left(r_{0}, r_{1}, r_{2}\right) b_{0} E_{r_{0}} b_{1} E_{r_{1}} b_{2} E_{r_{2}} .
\end{aligned}
$$

Let $k=e^{h / 2}$. While the arguments $b_{i}$ mentioned above are $\delta_{\mu} k$ or $\Delta k$, they are $\delta_{\mu}(\ln k)=\frac{1}{2} \delta_{\mu} h$ and $\Delta(\ln k)=\frac{1}{2} \Delta h$ in [8]. The second lemma gives the relations between these arguments.

Lemma B.2 If $g_{1}(y)=\frac{\sqrt{y}-1}{\ln y}$ and $g_{2}\left(y_{1}, y_{2}\right)=2 \frac{\sqrt{y_{1}}\left(\sqrt{y_{2}}-1\right) \ln y_{1}-\left(\sqrt{y_{1}}-1\right) \ln y_{2}}{\ln y_{1} \ln y_{2}\left(\ln y_{1}+\ln y_{2}\right)}$, then

$$
\begin{aligned}
\delta_{\mu} k & =k g_{1}(\boldsymbol{\Delta})\left[\delta_{\mu} h\right]=2 k g_{1}(\boldsymbol{\Delta})\left[\delta_{\mu} \ln k\right], \\
\Delta k & =k g_{1}(\boldsymbol{\Delta})[\Delta h]-g^{\mu \nu} k \mathbf{m} \circ g_{2}\left(\boldsymbol{\Delta}_{1}, \boldsymbol{\Delta}_{2}\right)\left[\left(\delta_{\mu} h\right) \otimes\left(\delta_{\nu} h\right)\right] \\
& =2 k g_{1}(\boldsymbol{\Delta})[\Delta \ln k]-4 g^{\mu \nu} k \mathbf{m} \circ g_{2}\left(\boldsymbol{\Delta}_{1}, \boldsymbol{\Delta}_{2}\right)\left[\left(\delta_{\mu} \ln k\right) \otimes\left(\delta_{\nu} \ln k\right)\right] .
\end{aligned}
$$

ProOF With $g_{1}(y):=\frac{1}{2} \int_{0}^{1} d s_{1} y^{s_{1} / 2}=(\sqrt{y}-1) \ln ^{-1} y$, we get

$$
\begin{aligned}
\delta_{\mu} k & =\delta_{\mu} e^{h / 2}=\int_{0}^{1} d s_{1} e^{\left(1-s_{1}\right) h / 2}\left(\delta_{\mu} h / 2\right) e^{s_{1} h / 2}=\frac{1}{2} k\left(\int_{0}^{1} d s_{1} \boldsymbol{\Delta}^{s_{1} / 2}\right)\left[\delta_{\mu} h\right] \\
& =k \frac{\boldsymbol{\Delta}^{1 / 2}-1}{\ln \boldsymbol{\Delta}}\left[\delta_{\mu} h\right]=2 k \frac{\boldsymbol{\Delta}^{1 / 2}-1}{\ln \boldsymbol{\Delta}}\left[\delta_{\mu} \ln k\right]=k g_{1}(\boldsymbol{\Delta})\left[\delta_{\mu} h\right]=2 k g_{1}(\boldsymbol{\Delta})\left[\delta_{\mu} \ln k\right] .
\end{aligned}
$$

Similarly for the Laplacian,

$$
\begin{aligned}
\Delta k= & -g^{\mu \nu}\left(\delta_{\mu} \delta_{\nu} k\right)=-\frac{1}{2} g^{\mu \nu} \delta_{\mu}\left[\int_{0}^{1} d s_{1} e^{\left(1-s_{1}\right) h / 2}\left(\delta_{\nu} h\right) e^{s_{1} h / 2}\right] \\
= & -\frac{1}{2} g^{\mu \nu}\left[\int_{0}^{1} d s_{1} e^{\left(1-s_{1}\right) h / 2}\left(\delta_{\mu} \delta_{\nu} h\right) e^{s_{1} h / 2}\right] \\
& -\frac{1}{4} g^{\mu \nu}\left[\int_{0}^{1} d s_{1} \int_{0}^{1-s_{1}} d s_{2} e^{\left(1-s_{1}-s_{2}\right) h / 2}\left(\delta_{\mu} h\right) e^{s_{2} h / 2}\left(\delta_{\nu} h\right) e^{s_{1} h / 2}\right] \\
& \left.-\frac{1}{4} g^{\mu \nu}\left[\int_{0}^{1} d s_{1} \int_{0}^{s_{1}} d s_{2} e^{\left(1-s_{1}\right) h / 2}\left(\delta_{\nu} h\right) e^{\left(s_{1}-s_{2}\right) h / 2} \delta_{\mu} h\right) e^{s_{2} h / 2}\right]
\end{aligned}
$$




$$
\begin{aligned}
= & -\frac{1}{2} g^{\mu \nu} k\left[\int_{0}^{1} d s_{1} \boldsymbol{\Delta}^{s_{1} / 2}\right]\left(\delta_{\mu} \delta_{\nu} h\right) \\
& -\frac{1}{4} g^{\mu \nu} k \mathbf{m} \circ\left[\int_{0}^{1} d s_{1} \int_{0}^{1-s_{1}} d s_{2} \boldsymbol{\Delta}_{1}^{\left(s_{1}+s_{2}\right) / 2} \boldsymbol{\Delta}_{2}^{s_{1} / 2}\right]\left[\left(\delta_{\mu} h\right) \otimes\left(\delta_{\nu} h\right)\right] \\
& -\frac{1}{4} g^{\mu \nu} k \mathbf{m} \circ\left[\int_{0}^{1} d s_{1} \int_{0}^{s_{1}} d s_{2} \boldsymbol{\Delta}_{1}^{s_{1} / 2} \boldsymbol{\Delta}_{2}^{s_{2} / 2}\right]\left[\left(\delta_{\mu} h\right) \otimes\left(\delta_{\nu} h\right)\right] \\
= & k g_{1}(\boldsymbol{\Delta})[\Delta h]-g^{\mu \nu} k \mathbf{m} \circ g_{2}\left(\boldsymbol{\Delta}_{1}, \boldsymbol{\Delta}_{2}\right)\left[\left(\delta_{\mu} h\right) \otimes\left(\delta_{\nu} h\right)\right]
\end{aligned}
$$

with

$$
\begin{aligned}
g_{2}\left(y_{1}, y_{2}\right) & :=\frac{1}{4} \int_{0}^{1} d s_{1} \int_{0}^{1-s_{1}} d s_{2} y_{1}^{\left(s_{1}+s_{2}\right) / 2} y_{2}^{s_{1} / 2}+\frac{1}{4} \int_{0}^{1} d s_{1} \int_{0}^{s_{1}} d s_{2} y_{1}^{s_{1} / 2} y_{2}^{s_{2} / 2} \\
& =2 \frac{\sqrt{y_{1}}\left(\sqrt{y_{2}}-1\right) \ln y_{1}-\left(\sqrt{y_{1}}-1\right) \ln y_{2}}{\ln y_{1} \ln y_{2}\left(\ln y_{1}+\ln y_{2}\right)} .
\end{aligned}
$$

The third lemma gives (technical) functional relations which allow a change of arguments inside our operators. Denote by $\mathbf{m}_{12}\left[b_{0} \otimes b_{1} \otimes b_{2}\right]:=b_{0} \otimes b_{1} b_{2}$ the partial multiplication.

Lemma B.3 For any operators like $f_{1}\left(R_{u}^{0}, \boldsymbol{\Delta}_{1}\right), f_{2}\left(R_{u}^{0}, \boldsymbol{\Delta}_{1}, \boldsymbol{\Delta}_{2}\right), g_{1}\left(\boldsymbol{\Delta}_{1}\right)$, and $g_{2}\left(\boldsymbol{\Delta}_{1}, \boldsymbol{\Delta}_{2}\right)$, one has

$$
\begin{aligned}
\mathbf{m} \circ f_{1}\left(R_{u}^{0}, \boldsymbol{\Delta}_{1}\right) \circ L_{k}^{1} \circ g_{1}\left(\boldsymbol{\Delta}_{1}\right) & =\mathbf{m} \circ R_{k}^{0} \circ f_{1}\left(R_{u}^{0}, \boldsymbol{\Delta}_{1}\right) \circ g_{1}\left(\boldsymbol{\Delta}_{1}\right), \\
\mathbf{m} \circ f_{1}\left(R_{u}^{0}, \boldsymbol{\Delta}_{1}\right) \circ \mathbf{m}_{12} \circ L_{k}^{1} \circ g_{2}\left(\boldsymbol{\Delta}_{1}, \boldsymbol{\Delta}_{2}\right) & =\mathbf{m} \circ R_{k}^{0} \circ f_{1}\left(R_{u}^{0}, \boldsymbol{\Delta}_{1} \boldsymbol{\Delta}_{2}\right) \circ g_{2}\left(\boldsymbol{\Delta}_{1}, \boldsymbol{\Delta}_{2}\right), \\
\mathbf{m} \circ f_{2}\left(R_{u}^{0}, \boldsymbol{\Delta}_{1}, \boldsymbol{\Delta}_{2}\right) \circ L_{k}^{1} \circ L_{k}^{2} \circ g_{2}\left(\boldsymbol{\Delta}_{1}, \boldsymbol{\Delta}_{2}\right) & =\mathbf{m} \circ R_{u}^{0} \circ \boldsymbol{\Delta}_{1}^{1 / 2} \circ f_{2}\left(R_{u}^{0}, \boldsymbol{\Delta}_{1}, \boldsymbol{\Delta}_{2}\right) \circ g_{2}\left(\boldsymbol{\Delta}_{1}, \boldsymbol{\Delta}_{2}\right) .
\end{aligned}
$$

Thus the operators on the LHS are respectively associated, modulo the multiplication operator $\mathbf{m}$, to operators defined by the spectral functions

$$
\sqrt{r_{0}} f_{1}\left(r_{0}, y_{1}\right) g_{1}\left(y_{1}\right), \quad \sqrt{r_{0}} f_{1}\left(r_{0}, y_{1} y_{2}\right) g_{2}\left(y_{1}, y_{2}\right), \quad r_{0} \sqrt{y_{1}} f_{2}\left(r_{0}, y_{1}, y_{2}\right) g_{2}\left(y_{1}, y_{2}\right),
$$

where $y_{1}, y_{2}$ belong to the spectrum of $\Delta$ and $r_{0}$ to the spectrum of $u$.

PROOF For the first relation, we compute the LHS on $b_{0} \otimes b_{1}$ using spectral decomposition:

$$
\begin{aligned}
\mathbf{m} \circ f_{1}\left(R_{u}^{0}, \boldsymbol{\Delta}_{1}\right) \circ L_{k}^{1} \circ g_{1}\left(\boldsymbol{\Delta}_{1}\right)\left[b_{0} \otimes b_{1}\right] & =\sum_{\substack{r_{0}, r_{1}, y, y_{1}}} f_{1}\left(r_{0}, y\right) \sqrt{r_{1}} g_{1}\left(y_{1}\right) b_{0} E_{r_{0}} E_{y}^{\boldsymbol{\Delta}}\left[E_{r_{1}} E_{y_{1}}^{\boldsymbol{\Delta}}\left(b_{1}\right)\right] \\
& =\sum_{\substack{r_{0}, r_{1}, y, y_{1}, z, z_{1}}} f_{1}\left(r_{0}, y\right) \sqrt{r_{1}} g_{1}\left(y_{1}\right) b_{0} E_{r_{0}} E_{z} E_{r_{1}} E_{z_{1}} b_{1} E_{y_{1} z_{1}} E_{y z}
\end{aligned}
$$

the projections products imply $r_{0}=z=r_{1}=z_{1}$ and $y_{1} z_{1}=y z$, so this is equal to

$$
\begin{aligned}
\sum_{\substack{r_{0}, y \\
z}} f_{1}\left(r_{0}, y\right) \sqrt{r_{0}} g_{1}(y) b_{0} E_{r_{0}} E_{z} b_{1} E_{y z} & =\sum_{\substack{r_{0}, y \\
z}} \sqrt{r_{0}} f_{1}\left(r_{0}, y\right) g_{1}(y) b_{0} E_{r_{0}} E_{y}^{\Delta}\left(b_{1}\right) \\
& =\mathbf{m} \circ R_{k}^{0} \circ f_{1}\left(R_{u}^{0}, \boldsymbol{\Delta}_{1}\right) \circ g_{1}\left(\boldsymbol{\Delta}_{1}\right)\left[b_{0} \otimes b_{1}\right] .
\end{aligned}
$$

For the second relation, we compute the LHS on $b_{0} \otimes b_{1} \otimes b_{2}$ :

$$
\begin{aligned}
\mathbf{m} \circ f_{1}\left(R_{u}^{0}, \boldsymbol{\Delta}_{1}\right) \circ \mathbf{m}_{12} \circ L_{k}^{1} \circ g_{2}\left(\boldsymbol{\Delta}_{1}, \boldsymbol{\Delta}_{2}\right)\left[b_{0} \otimes b_{1} \otimes b_{2}\right] \\
=\sum_{\substack{r_{0}, r_{1}, y, y_{1}, y_{2}}} f_{1}\left(r_{0}, y\right) \sqrt{r_{1}} g_{2}\left(y_{1}, y_{2}\right) b_{0} E_{r_{0}} E_{y}^{\boldsymbol{\Delta}}\left[E_{r_{1}} E_{y_{1}}^{\boldsymbol{\Delta}}\left(b_{1}\right) E_{y_{2}}^{\boldsymbol{\Delta}}\left(b_{2}\right)\right]
\end{aligned}
$$




$$
=\sum_{\substack{r_{0}, r_{1}, y_{y 1}, y_{2}, z, z_{1}, z_{2}}} f_{1}\left(r_{0}, y\right) \sqrt{r_{1}} g_{2}\left(y_{1}, y_{2}\right) b_{0} E_{r_{0}} E_{z} E_{r_{1}} E_{z_{1}} b_{1} E_{y_{1} z_{1}} E_{z_{2}} b_{2} E_{y_{2} z_{2}} E_{y z}
$$

which implies $r_{0}=z=r_{1}=z_{1}, y_{1} z_{1}=z_{2}$, and $y_{2} z_{2}=y z$, and

$$
\begin{aligned}
& =\sum_{\substack{r_{0}, z_{1}, z_{2} \\
y_{1}, y_{2}}} f\left(r_{0}, y_{1} y_{2}\right) \sqrt{r_{0}} g_{2}\left(y_{1}, y_{2}\right) b_{0} E_{r_{0}} E_{z_{1}} b_{1} E_{y_{1} z_{1}} E_{z_{2}} b_{2} E_{y_{2} z_{2}} \\
& =\sum_{r_{0}, y_{1}, y_{2}} \sqrt{r_{0}} f\left(r_{0}, y_{1} y_{2}\right) g_{2}\left(y_{1}, y_{2}\right) b_{0} E_{r_{0}} E_{y_{1}}^{\boldsymbol{\Delta}}\left(b_{1}\right) E_{y_{2}}^{\boldsymbol{\Delta}}\left(b_{2}\right) \\
& =\mathbf{m} \circ R_{k}^{0} \circ f\left(R_{u}^{0}, \boldsymbol{\Delta}_{1} \boldsymbol{\Delta}_{2}\right) \circ g_{2}\left(\boldsymbol{\Delta}_{1}, \boldsymbol{\Delta}_{2}\right)\left[b_{0} \otimes b_{1} \otimes b_{2}\right] .
\end{aligned}
$$

For the third relation, we compute the LHS on $b_{0} \otimes b_{1} \otimes b_{2}$ :

$$
\begin{aligned}
\mathbf{m} & \circ f\left(R_{u}^{0}, \boldsymbol{\Delta}_{1}, \boldsymbol{\Delta}_{2}\right) \circ L_{k}^{1} \circ L_{k}^{2} \circ g_{2}\left(\boldsymbol{\Delta}_{1}, \boldsymbol{\Delta}_{2}\right)\left[b_{0} \otimes b_{1} \otimes b_{2}\right] \\
& =\sum_{\substack{r_{0}, r_{1}, r_{2}, y_{1}, y_{2}, y_{1}^{\prime}, y_{2}^{\prime}}} f_{2}\left(r_{0}, y_{1}, y_{2}\right) \sqrt{r_{1}} \sqrt{r_{2}} g_{2}\left(y_{1}^{\prime}, y_{2}^{\prime}\right) b_{0} E_{r_{0}} E_{y_{1}}^{\boldsymbol{\Delta}}\left[E_{r_{1}} E_{y_{1}^{\prime}}^{\Delta}\left(b_{1}\right)\right] E_{y_{2}}^{\Delta}\left[E_{r_{2}} E_{y_{2}^{\prime}}^{\boldsymbol{\Delta}}\left(b_{2}\right)\right] \\
= & \sum_{\substack{r_{0}, r_{1}, r_{2}, y_{1}, y_{2}, y_{1}^{\prime}, y_{2}^{\prime}, z_{1}, z_{2}, z_{1}^{\prime}, z_{2}^{\prime}}} f_{2}\left(r_{0}, y_{1}, y_{2}\right) \sqrt{r_{1}} \sqrt{r_{2}} g_{2}\left(y_{1}^{\prime}, y_{2}^{\prime}\right) b_{0} E_{r_{0}} E_{z_{1}} E_{r_{1}} E_{z_{1}^{\prime}} b_{1} E_{y_{1}^{\prime} z_{1}^{\prime}} E_{y_{1} z_{1}} E_{z_{2}} E_{r_{2}} E_{z_{2}^{\prime}} b_{2} E_{y_{2}^{\prime} z_{2}^{\prime}} E_{y_{2} z_{2}}
\end{aligned}
$$

which implies $r_{0}=z_{1}=r_{1}=z_{1}^{\prime}, y_{1}^{\prime} z_{1}^{\prime}=y_{1} z_{1}=z_{2}=r_{2}=z_{2}^{\prime}$, and $y_{2}^{\prime} z_{2}^{\prime}=y_{2} z_{2}$, so that:

$$
\begin{aligned}
& =\sum_{\substack{r_{0}, z_{1}, z_{2} \\
y_{1}, y_{2}}} f_{2}\left(r_{0}, y_{1}, y_{2}\right) \sqrt{r_{0}} \sqrt{y_{1} r_{0}} g_{2}\left(y_{1}, y_{2}\right) b_{0} E_{r_{0}} E_{z_{1}} b_{1} E_{y_{1} z_{1}} E_{z_{2}} b_{2} E_{y_{2} z_{2}} \\
& =\sum_{r_{0}, y_{1}, y_{2}} r_{0} \sqrt{y_{1}} f_{2}\left(r_{0}, y_{1}, y_{2}\right) g_{2}\left(y_{1}, y_{2}\right) b_{0} E_{r_{0}} E_{y_{1}}^{\boldsymbol{\Delta}}\left(b_{1}\right) E_{y_{2}}^{\boldsymbol{\Delta}}\left(b_{2}\right) \\
& =\mathbf{m} \circ R_{u}^{0} \circ \boldsymbol{\Delta}_{1}^{1 / 2} \circ f_{2}\left(R_{u}^{0}, \boldsymbol{\Delta}_{1}, \boldsymbol{\Delta}_{2}\right) \circ g_{2}\left(\boldsymbol{\Delta}_{1}, \boldsymbol{\Delta}_{2}\right)\left[b_{0} \otimes b_{1} \otimes b_{2}\right] .
\end{aligned}
$$

We can now change (5.7) in order to compare with [8, Theorem 5.2]. As in Lemma B.1, let

$$
f_{\Delta k}\left(r_{0}, y_{1}\right):=F_{\Delta k}\left(r_{0}, r_{0} y_{1}\right), \quad f_{\partial k \partial k}^{\mu \nu}\left(r_{0}, y_{1}, y_{2}\right):=F_{\partial k \partial k}^{\mu \nu}\left(r_{0}, r_{0} y_{1}, r_{0} y_{1} y_{2}\right)
$$

Using Lemmas B.2 and B.3, one gets

$$
\begin{aligned}
\mathbf{m} \circ & F_{\Delta k}\left(R_{u}^{0}, R_{u}^{1}\right)[a \otimes \Delta k]=\mathbf{m} \circ f_{\Delta k}\left(R_{u}^{0}, \boldsymbol{\Delta}_{1}\right)[a \otimes \Delta k] \\
= & \mathbf{m} \circ f_{\Delta k}\left(R_{u}^{0}, \boldsymbol{\Delta}_{1}\right) \circ L_{k}^{1} \circ g_{1}\left(\boldsymbol{\Delta}_{1}\right)[a \otimes \Delta h] \\
& \quad-g^{\mu \nu} \mathbf{m} \circ f_{\Delta k}\left(R_{u}^{0}, \boldsymbol{\Delta}_{1}\right) \circ L_{k}^{1} \circ \mathbf{m}_{12} \circ g_{2}\left(\boldsymbol{\Delta}_{1}, \boldsymbol{\Delta}_{2}\right)\left[a \otimes\left(\delta_{\mu} h\right) \otimes\left(\delta_{\nu} h\right)\right] \\
= & \mathbf{m} \circ R_{k}^{0} \circ f_{\Delta k}\left(R_{u}^{0}, \boldsymbol{\Delta}_{1}\right) \circ g_{1}\left(\boldsymbol{\Delta}_{1}\right)[a \otimes \Delta h] \\
& \quad-g^{\mu \nu} \mathbf{m} \circ R_{k}^{0} \circ f_{\Delta k}\left(R_{u}^{0}, \boldsymbol{\Delta}_{1}\right) \circ g_{2}\left(\boldsymbol{\Delta}_{1}, \boldsymbol{\Delta}_{2}\right)\left[a \otimes\left(\delta_{\mu} h\right) \otimes\left(\delta_{\nu} h\right)\right] \\
= & 2 \mathbf{m} \circ R_{k}^{0} \circ f_{\Delta k}\left(R_{u}^{0}, \boldsymbol{\Delta}_{1}\right) \circ g_{1}\left(\boldsymbol{\Delta}_{1}\right)[a \otimes \Delta \ln k] \\
& \quad-4 g^{\mu \nu} \mathbf{m} \circ R_{k}^{0} \circ f_{\Delta k}\left(R_{u}^{0}, \boldsymbol{\Delta}_{1} \boldsymbol{\Delta}_{2}\right) \circ g_{2}\left(\boldsymbol{\Delta}_{1}, \boldsymbol{\Delta}_{2}\right)\left[a \otimes\left(\delta_{\mu} \ln k\right) \otimes\left(\delta_{\nu} \ln k\right)\right]
\end{aligned}
$$

and

$$
\begin{aligned}
& \mathbf{m} \circ F_{\partial k \partial k}^{\mu \nu}\left(R_{u}^{0}, R_{u}^{1}, R_{u}^{2}\right)\left[a \otimes\left(\delta_{\mu} k\right) \otimes\left(\delta_{\nu} k\right)\right]=\mathbf{m} \circ f_{\partial k \partial k}^{\mu \nu}\left(R_{u}^{0}, \boldsymbol{\Delta}_{1}, \boldsymbol{\Delta}_{2}\right)\left[a \otimes\left(\delta_{\mu} k\right) \otimes\left(\delta_{\nu} k\right)\right] \\
& \quad=\mathbf{m} \circ f_{\partial k \partial k}^{\mu \nu}\left(R_{u}^{0}, \boldsymbol{\Delta}_{1}, \boldsymbol{\Delta}_{2}\right) \circ L_{k}^{1} \circ L_{k}^{2} \circ g_{1}\left(\boldsymbol{\Delta}_{1}\right) \circ g_{1}\left(\boldsymbol{\Delta}_{2}\right)\left[a \otimes\left(\delta_{\mu} h\right) \otimes\left(\delta_{\nu} h\right)\right]
\end{aligned}
$$




$$
\begin{aligned}
& =\mathbf{m} \circ R_{u}^{0} \circ \boldsymbol{\Delta}_{1}^{1 / 2} \circ f_{\partial k \partial k}^{\mu \nu}\left(R_{u}^{0}, \boldsymbol{\Delta}_{1}, \boldsymbol{\Delta}_{2}\right) \circ g_{1}\left(\boldsymbol{\Delta}_{1}\right) \circ g_{1}\left(\boldsymbol{\Delta}_{2}\right)\left[a \otimes\left(\delta_{\mu} h\right) \otimes\left(\delta_{\nu} h\right)\right] \\
& =4 \mathbf{m} \circ R_{u}^{0} \circ \boldsymbol{\Delta}_{1}^{1 / 2} \circ f_{\partial k \partial k}^{\mu \nu}\left(R_{u}^{0}, \boldsymbol{\Delta}_{1}, \boldsymbol{\Delta}_{2}\right) \circ g_{1}\left(\boldsymbol{\Delta}_{1}\right) \circ g_{1}\left(\boldsymbol{\Delta}_{2}\right)\left[a \otimes\left(\delta_{\mu} \ln k\right) \otimes\left(\delta_{\nu} \ln k\right)\right] .
\end{aligned}
$$

So, the sum gives

$$
\begin{aligned}
& \mathbf{m} \circ F_{\Delta k}\left(R_{u}^{0}, R_{u}^{1}\right)[a \otimes \Delta k]+\mathbf{m} \circ F_{\partial k \partial k}^{\mu \nu}\left(R_{u}^{0}, R_{u}^{1}, R_{u}^{2}\right)\left[a \otimes\left(\delta_{\mu} k\right) \otimes\left(\delta_{\nu} k\right)\right] \\
& =\mathbf{m} \circ G_{(\Delta \ln k)}\left(R_{u}^{0}, \Delta_{1}\right)[a \otimes \Delta \ln k]+\mathbf{m} \circ G_{(\partial \ln k)(\partial \ln k)}^{\mu \nu}\left(R_{u}^{0}, \Delta_{1}, \Delta_{2}\right)\left[a \otimes\left(\delta_{\mu} \ln k\right) \otimes\left(\delta_{\nu} \ln k\right)\right]
\end{aligned}
$$

with

$$
\begin{aligned}
G_{(\Delta \ln k)}\left(R_{u}^{0}, \boldsymbol{\Delta}_{1}\right):= & 2 R_{k}^{0} \circ f_{\Delta k}\left(R_{u}^{0}, \boldsymbol{\Delta}_{1}\right) \circ g_{1}\left(\boldsymbol{\Delta}_{1}\right), \\
G_{(\partial \ln k)(\partial \ln k)}^{\mu \nu}\left(R_{u}^{0}, \boldsymbol{\Delta}_{1}, \boldsymbol{\Delta}_{2}\right):= & 4 R_{u}^{0} \circ \boldsymbol{\Delta}_{1}^{1 / 2} \circ f_{\partial k \partial k}^{\mu \nu}\left(R_{u}^{0}, \boldsymbol{\Delta}_{1}, \boldsymbol{\Delta}_{2}\right) \circ g_{1}\left(\boldsymbol{\Delta}_{1}\right) \circ g_{1}\left(\boldsymbol{\Delta}_{2}\right) \\
& -4 g^{\mu \nu} R_{k}^{0} \circ f_{\Delta k}\left(R_{u}^{0}, \boldsymbol{\Delta}_{1} \boldsymbol{\Delta}_{2}\right) \circ g_{2}\left(\boldsymbol{\Delta}_{1}, \boldsymbol{\Delta}_{2}\right) .
\end{aligned}
$$

The associated spectral functions are

$$
\begin{aligned}
G_{(\Delta \ln k)}\left(r_{0}, y_{1}\right) & =2 \sqrt{r_{0}} f_{\Delta k}\left(r_{0}, y_{1}\right) g_{1}\left(y_{1}\right), \\
G_{(\partial \ln k)(\partial \ln k)}^{\mu \nu}\left(r_{0}, y_{1}, y_{2}\right) & =4 r_{0} \sqrt{y_{1}} f_{\partial k \partial k}^{\mu \nu}\left(r_{0}, y_{1}, y_{2}\right) g_{1}\left(y_{1}\right) g_{1}\left(y_{2}\right)-4 g^{\mu \nu} \sqrt{r_{0}} f_{\Delta k}\left(r_{0}, y_{1} y_{2}\right) g_{2}\left(y_{1}, y_{2}\right) .
\end{aligned}
$$

Another change of convention concerns the derivations of $C^{\infty}\left(\mathbb{T}_{\Theta}^{2}\right):$ in $[8], \widehat{\delta}_{\mu}:=-i \delta_{\mu}$ is used. This implies that their expressions like $\left(\widehat{\delta}_{\mu} \ln k\right)\left(\widehat{\delta}_{\nu} \ln k\right)$ correspond to our $-\left(\delta_{\mu} \ln k\right) \otimes\left(\delta_{\nu} \ln k\right)$. Notice also their combination $\widehat{\delta}_{1}^{2} \ln k+|\tau|^{2} \widehat{\delta}_{2}^{2} \ln k+2 \tau_{1} \tau_{2} \widehat{\delta}_{1} \widehat{\delta}_{2} \ln k=g^{\mu \nu} \widehat{\delta}_{\mu} \widehat{\delta}_{\nu} \ln k=-g^{\mu \nu} \delta_{\mu} \delta_{\nu} \ln k=\Delta \ln k$. Thus for a comparison of the two results, a $-\operatorname{sign}$ has to be taken into account for the $G_{(\partial \ln k)(\partial \ln k)}^{\mu \nu}$ term. Finally, [8, Theorem 5.2] is written in terms of functions of $\ln \boldsymbol{\Delta}$, thus it remains to make the final change of variables $y_{1}=e^{x}$ in $G_{(\Delta \ln k)}$, and $y_{1}=e^{s}$ and $y_{2}=e^{t}$ in $G_{(\partial \ln k)(\partial \ln k)}^{\mu \nu}$.

In [8, Theorem 5.2], $\mathcal{R}_{F K}=-\frac{\pi}{\tau_{2}} \times$ [expression in $\left.R_{1}, R_{2}, W\right]$ while in (5.7), one has written $\mathcal{R}_{2}=\frac{1}{4 \pi} \times$ [expression in $\left.F_{\Delta k}, F_{\partial k \partial k}^{\mu \nu}\right]$. The proof that $\mathcal{R}_{2}=\frac{\tau_{2}}{(2 \pi)^{2}} \mathcal{R}_{F K}$ is then equivalent to check that [expression in $\left.R_{1}, R_{2}, W\right]=-$ [expression in $F_{\Delta k}, F_{\partial k \partial k}^{\mu \nu}$. The previous technical results imply that this is equivalent to show that

$$
\begin{aligned}
R_{1}(x) & =-G_{(\Delta \ln k)}\left(r_{0}, e^{x}\right), \\
R_{2}(s, t) & =G_{(\partial \ln k)(\partial \ln k)}^{11}\left(r_{0}, e^{s}, e^{t}\right), \\
|\tau|^{2} R_{2}(s, t) & =G_{(\partial \ln k)(\partial \ln k)}^{22}\left(r_{0}, e^{s}, e^{t}\right), \\
\tau_{1} R_{2}(s, t)-i \tau_{2} W(s, t) & =G_{(\partial \ln k)(\partial \ln k)}^{12}\left(r_{0}, e^{s}, e^{t}\right), \\
\tau_{1} R_{2}(s, t)+i \tau_{2} W(s, t) & =G_{(\partial \ln k)(\partial \ln k)}^{21}\left(r_{0}, e^{s}, e^{t}\right) .
\end{aligned}
$$

All these relations can be checked directly. In particular, the relations on the RHS are independent of the variable $r_{0}$.

In order to compare (5.8) for the noncommutative four torus with [11, Theorem 5.4], we can use the results in [14]. As before, we need the correspondence (A.4) between our trace $\varphi$ and their trace $\varphi_{0} \equiv \mathfrak{t}$. Here $g_{i}^{\mu \nu}=\delta^{\mu \nu}$ on the base tori $\mathbb{T}_{B, i}^{2}$, so that $\left|g_{i}\right|^{1 / 2}=1$. Denote by $\mathcal{R}_{F K}$ the curvature obtained in [11, Theorem 5.4], which is $\pi^{2}$ times [11, eq. (5.1)]. A comparison between eq. (1) and (3) in [14] and [11, eq. (5.1)] gives

$$
\varphi_{0}\left(a \mathcal{R}_{F K}\right)=\frac{1}{2} \varphi_{0}\left(a \int_{\mathbb{S}^{3}} b_{2}(\xi) d \Omega\right)=\frac{\pi^{2}}{2} \varphi_{0}\left(a\left[-\delta^{\mu \nu} k^{-2}\left(\widehat{\delta}_{\mu} \widehat{\delta}_{\nu} k^{2}\right) k^{-2}+\frac{3}{2} \delta^{\mu \nu} k^{-2}\left(\widehat{\delta}_{\mu} k^{2}\right) k^{-2}\left(\widehat{\delta}_{\nu} k^{2}\right) k^{-2}\right]\right)
$$




$$
=\frac{\pi^{2}}{2(2 \pi)^{4}} \varphi\left(a\left[\delta^{\mu \nu} k^{-2}\left(\delta_{\mu} \delta_{\nu} k^{2}\right) k^{-2}-\frac{3}{2} \delta^{\mu \nu} k^{-2}\left(\delta_{\mu} k^{2}\right) k^{-2}\left(\delta_{\nu} k^{2}\right) k^{-2}\right]\right)=\varphi\left(a \mathcal{R}_{2}\right),
$$

and the two results coincide. ${ }^{2}$

\section{References}

\section{References}

[1] B. Iochum, T. Masson, Heat trace for Laplace type operators with non-scalar symbols, Journal of Geometry and Physics 116 (2017) 90-118.

[2] I. G. Avramidi, Gauged gravity via spectral asymptotics of non-Laplace type operators, Journal of High Energy Physics 2004 (07) (2004) 030.

[3] I. G. Avramidi, T. P. Branson, Heat kernel asymptotics of operators with non-Laplace principal part, Reviews in Mathematical Physics 13 (07) (2001) 847-890.

[4] Wolfram Research Inc., Mathematica, Version 11.1, 2017.

[5] B. Iochum, T. Masson, Heat asymptotics for nonminimal Laplace type operators and application to noncommutative tori, Mathematica Notebook added as ancillary file on arXiv, 2017.

[6] A. Connes, P. Tretkoff, The Gauss-Bonnet theorem for the noncommutative two torus, Noncommutative Geometry, Arithmetic, and Related Topics, Johns Hopkins Univ. Press, Baltimore, MD (2011) 141-158.

[7] A. Connes, H. Moscovici, Modular curvature for noncommutative two-tori, Journal of the American Mathematical Society 27 (3) (2014) 639-684.

[8] F. Fathizadeh, M. Khalkhali, Scalar curvature for the noncommutative two torus, Journal of Noncommutative Geometry 7 (2013) 1145-1183.

[9] F. Fathizadeh, M. Khalkhali, The Gauss-Bonnet theorem for noncommutative two tori with a general conformal structure, Journal of Noncommutative Geometry 6 (2012) 457-480.

[10] L. Dabrowski, A. Sitarz, Curved noncommutative torus and Gauss-Bonnet, Journal of Mathematical Physics 54 (2013) 013518.

[11] F. Fathizadeh, M. Khalkhali, Scalar curvature for noncommutative four-tori, Journal of Noncommutative Geometry 9 (2015) 473-503.

[12] S. Azzali, C. Lévy, C. Neira-Jiménez, S. Paycha, Traces of holomorphic families of operators on the noncommutative torus and on Hilbert Modules, in: Geometrics methods in Physics, Birkhäuser, 3-38, 2014.

[13] A. Sitarz, Wodzicki residue and minimal operators on a noncommutative 4-dimensional torus, J. Pseudo-Differ. Oper. Appl. 5 (2014) 305-317.

\footnotetext{
${ }^{2}$ The constant $c$ computed in $\left[14\right.$, eq. (3)] is not $c=1 /\left(2 \pi^{2}\right)$ as claimed but $c=1 / 2$ as shown by a direct comparison between $\left[14\right.$, eq. (3)] and [11, eq. (5.1)] where a factor $\pi^{2}$ is not written. This explains the factor $\frac{1}{2}$ in our first equality.
} 
[14] F. Fathizadeh, On the scalar curvature for the noncommutative four torus, Journal of Mathematical Physics 56 (6) (2015) 062303.

[15] L. Dabrowski, A. Sitarz, An asymmetric noncommutative torus, SIGMA 11 (2015) 075, 11 pages.

[16] Y. Liu, Modular curvature for toric noncommutative manifolds, arXiv:1510.04668v2 [math.OA], 2015.

[17] S. Sadeghi, On logarithmic Sobolev inequality and a scalar curvature formula for noncommutative tori, Ph.D. thesis, Western University, Ontario, 2016.

[18] A. Connes, F. Fathizadeh, The term $a_{4}$ in the heat kernel expansion of noncommutative tori, arXiv:1611.09815v1 [math.QA], 2016.

[19] A. Connes, C*-algèbres et géométrie différentielle, C. R. Acad. Sc. Paris - Série A 290 (1980) 599-604.

[20] M. Lesch, H. Moscovici, Modular curvature and Morita equivalence, Geom. Funct. Anal. 26 (2016) 818-873.

[21] G. K. Pedersen, On the Operator Equation $H T+T H=2 K$, Indiana University Mathematics Journal 25 (11) (1976) 1029-1033.

[22] P. B. Gilkey, Invariance theory, the heat equation and the Atiyah-Singer index theorem, Studies in Advanced Mathematics, CRC Press, Inc, 2 edn., 1995.

[23] P. B. Gilkey, Asymptotic formulae in spectral geometry, CRC press, 2003.

[24] J. M. G. Fell, The structure of algebras of operator fields, Acta Mathematica 106 (3-4) (1961) $233-280$.

[25] B. Blackadar, Operator algebras: theory of $C^{*}$-algebras and von Neumann algebras, vol. 122, Springer Science, 2006.

[26] J. M. Gracia-Bondía, J. C. Várilly, H. Figueroa, Elements of Noncommutative Geometry, Birkhäuser Boston, 2001.

[27] M. Dubois-Violette, A. Kriegl, Y. Maeda, P. W. Michor, Smooth *-algebras, Progress of Theoretical Physics Supplement 144 (2002) 54-78.

[28] M. Lesch, Divided differences in noncommutative geometry: Rearrangement lemma, functional calculus and expansional formula, Journal of Noncommutative Geometry 11 (2017) 193-223. 